\title{
Large deviations for infectious diseases models
}

\author{
Peter Kratz and Etienne Pardoux*
}

August 28, 2018

\section{Introduction}

Consider a model of infectious disease dynamics where the total number of individuals is constant over time, equal to $N$, and we denote by $Z^{N}(t)$ the vector of proportions of this population in each compartment (susceptible, infectious, removed, etc.). Our probabilistic model takes into account each event of infection, removal, etc. It takes the form

$$
Z^{N, x}(t):=Z^{N}(t):=x+\frac{1}{N} \sum_{j=1}^{k} h_{j} P_{j}\left(\int_{0}^{t} N \beta_{j}\left(Z^{N}(s)\right) d s\right) .
$$

Here, $\left(P_{j}\right)_{1 \leq j \leq k}$ are i.i.d. standard Poisson processes. The $h_{j} \in \mathbb{Z}^{d}$ denote the $k$ respective jump directions with jump rates $\beta_{j}(x)$ and $x \in A$ (where $A$ is the "domain" of the process). The $d$ components of the process denote the "proportions" of individuals in the various compartments. Usually $A$ is a compact or at least a bounded set. For example, in the models we have in mind the compartment sizes are non-negative, hence $A \subset \mathbb{R}_{+}^{d}$.

As we shall prove below, $Z_{t}^{N, x} \rightarrow Y_{t}^{x}$ as $N \rightarrow \infty$, where $Y_{t}^{x}$ is the solution of the ODE

$$
Y^{x}(t):=Y(t):=x+\int_{0}^{t} b\left(Y^{x}(s)\right) d s
$$

with

$$
b(z):=\sum_{j=1}^{k} \beta_{j}(z) h_{j}
$$

This Law of Large Numbers result goes back to Kurtz [1978] (see the version in Theorem 3.1 below, where a rate of convergence is given).

Most of the literature on mathematical models of disease dynamics treats deterministic models of the type of (2). When an epidemics is established, and each compartment of the model contains a significant proportion of the total population, if $N$ is large enough, the ODE (2) is a good model for the epidemics. The original stochastic model (11), which we

*Aix-Marseille Université, CNRS, Centrale Marseille, I2M, UMR 7373, 13453 Marseille, France, e-mail adress: etienne.pardoux@univ-amu.fr 
believe to be more realistic than the (2), can be considered as a stochastic perturbation of (2). However, we know from the work of Freidlin and Wentzell [2012], that small Brownian perturbations of an ODE will eventually produce a large deviation from its law of large numbers limit. For instance, if the ODE starts in the basin of attraction of an locally stable equilibrium, the solution of the ODE converges to that equilibrium, and stays for ever close to that equilibrium. The theory of Freidlin and Wentzell, based upon the theory of Large Deviations, predicts that soon or later the solution of a random perturbation of that ODE will exit the basin of attraction of the equilibrium. The aim of this paper is to show that the Poissonian perturbation (11) of (2) behaves similarly. This should allow us to predicts the time taken by an endemic equilibrium to cease, and a disease-free equilibrium to replace it.

We shall apply at the end of this paper our results to the following example.

Example 1.1. We consider a so-called SIRS model without demography $(S(t)$ being the number of susceptible individuals, $I(t)$ the number of infectious individuals and $R(t)$ the number of removed/immune individuals at time $t)$. We let $\beta>0$ and assume that the average number of new infections per unit time is $\beta S(t) I(t) / N \mathbb{1}$ For $\gamma, \nu>0$, we assume that the average number of recoveries per unit time is $\gamma I(t)$ and the average number of individuals who lose immunity is $\nu R(t)$. As population size is constant, we can reduce the dimension of the model by solely considering the proportion of infectious and removed at time t. Using the notation of equations (11) and (2), we have

$$
\begin{aligned}
& A=\left\{x \in \mathbb{R}_{+}^{2} \mid 0 \leq x_{1}+x_{2} \leq 1\right\}, \quad h_{1}=(1,0)^{\top}, \quad h_{2}=(-1,1)^{\top}, \quad h_{3}=(0,-1)^{\top}, \\
& \beta_{1}(z)=\beta z_{1}\left(1-z_{1}-z_{2}\right), \quad \beta_{2}(z)=\gamma z_{1}, \quad \beta_{3}(z)=\nu z_{2} .
\end{aligned}
$$

It is easy to see that in this example the ODE (2) has a disease free equilibrium $\bar{x}=(0,0)^{\top}$. This equilibrium is asymptotically stable if $R_{0}=\beta / \gamma<1 . R_{0}$ is the so-called basic reproduction number. It denotes the average number of secondary cases infected by one primary case during its infectious period at the start of the epidemic (while essentially everybody is susceptible). If $R_{0}>1, \bar{x}$ is unstable and there exists a second, endemic equilibrium $x^{*}=\left(\frac{\nu(\beta-\gamma)}{\beta(\gamma+\nu)}, \frac{\gamma(\beta-\gamma)}{\beta(\gamma+\nu}\right)$ which is asymptotically stable. While in the deterministic model the proportion of infectious and removed individuals converges to the endemic equilibrium $x^{*}$, the disease will go extinct soon or later in the stochastic model.

Our results also apply to other models like the $S I V$ model ( $V$ like vaccinated) and the $S_{0} I S_{1}$ (with two levels of susceptibility), see Kratz et al. [2015] and the references therein. These two models have the property that for certain values of their parameters, both the disease-free equilibrium and one of the endemic equilibria are locally stable. Our results predict the time taken by the solution of the stochastic equation to leave the basin of attraction of the endemic equilibrium. We shall discuss those and other applications elsewhere in the future.

There is already a vast literature on the theory of large deviations for systems with Poissonian inputs, see Dupuis and Ellis [1997], Dupuis et al. [1991], Feng and Kurtz [2006], Shwartz and Weiss [1995], among others.

\footnotetext{
${ }^{1}$ The reasoning behind this is the following. Assume that an infectious individuals meets on average $\alpha>0$ other individuals in unit time. If each contact of a susceptible and an infectious individual yields a new infection with probability $p$, the average number of new infections per unit time is $\beta S(t) I(t) / N$, where $\beta=p \alpha$ since all individuals are contacted with the same probability (hence $S(t) / N$ is the probability that a contacted individual is susceptible).
} 


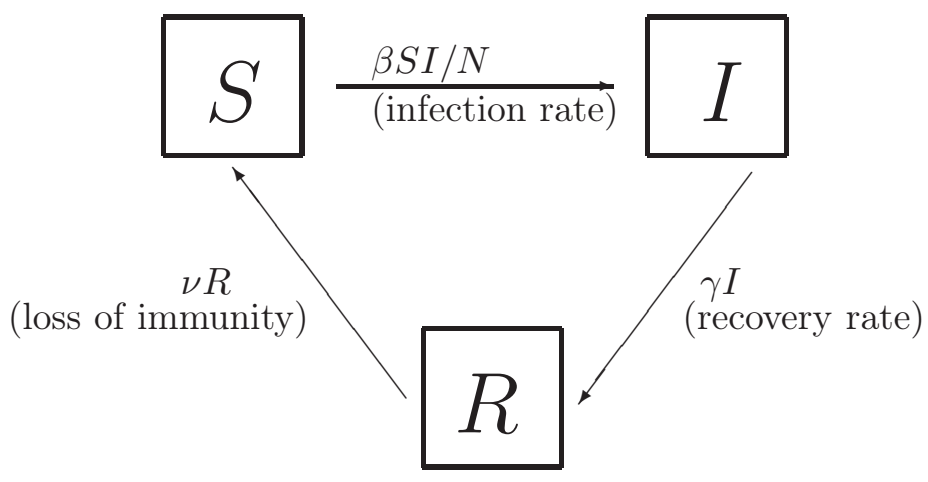

Figure 1: Transmission diagram of the SIRS model without demography.

However, the assumptions in those works are not satisfied in our case. The difficulty is the following. For obvious reasons, the solution of our SDE (11) must remain in $\mathbb{R}_{+}^{d}$. This implies that some rates vanish when one of the components of $Z^{N, x}(t)$ vanishes. However, the expression of the large deviation fucntional (as well as the ratio of the probabilities in the Girsanov theorem) involves the logarithm of those rates, which hence explodes as the rate vanishes. The same happens with the computer network models which was the motivation of the work of Shwartz and Weiss 1995], and this led them to consider situations with vanishing rates in Shwartz and Weiss [2005]. However, even the assumptions in that paper are not fully satisfied in our models (see our discussion below in section 2.2). For that reason, in order to avoid the ackward situation where we would have to cite both Shwartz and Weiss [1995] and Shwartz and Weiss [2005], and add some arguments to cope with our specific situation, we prefered to rewrite the whole theory, so as to cover the situation of the epidemiological models in a self-consistent way. We must however recognize that the work of Shwartz and Weiss has been an importance source of inspiration for this work.

Let us now discuss one subtlety of our models. In the models without demography, i.e. the models where the total population remains constant, then we choose $N$ as this total population, so that the various components of the vector $Z_{t}^{N, x}$ are the proportions of the total population in the various compartments of the model, that is each component of $Z_{t}^{N, x}$ at any time is of the form $k / N$, where $k \in \mathbb{Z}_{+}$, and also $\sum_{i=1}^{d} Z_{i}^{N, x}(t)=1$, if $Z_{i}^{N, x}(t)$ denotes the $i$-th component of the vector $Z^{N, x}(t), 1 \leq i \leq d$. In this case, provided we start our SDE from a point of the type $\left(k_{1} / N, \ldots, k_{d} / N\right)$, where $k_{1}, \ldots, k_{d} \in \mathbb{Z}_{+}$, then the solution visits only such points, and cannot escape the set $\mathbb{R}_{+}^{d}$ without hitting first its boundary, where the rates for exiting vanish. Consequently $Z_{t}^{N, x}$ remains in $\mathbb{R}_{+}^{d}$ for all time a.s. However, if we start our process outside the above grid, or if the total population size does not remain constant, the components of the vector $Z_{t}^{N, x}$ multiplied by $N$ need not be integers. Then some of the components of $Z_{t}^{N, x}$ might become negative, and one can still continue to define $Z_{t}^{N, x}$ provided for any $1 \leq i \leq d$ and $1 \leq j \leq k$, the rule $x_{i}=0 \Rightarrow \beta_{j}(x)=0$ is extended to $x_{i} \leq 0 \Rightarrow \beta_{j}(x)=0$. However, in order to make things simpler, we restrict ourselves in this paper to the situation where all coordinates of the vector $N Z_{0}^{N, x}$ are integers, and the same is true with $N Z_{t}^{N, x}$ for all $t>0$. In particular, we shall consider equation (10) only with a starting point $x$ such that all coordinates of $N x$ are integers. This will be explicitly recalled in the main statements, and implicitly 
assumed everywhere. We shall consider more general situations in a further publication.

The paper is organized as follows Our set-up is made precise and the general assumptions are formulated in section 2 . Section 3 is devoted to the law of large numbers. In section 4 we study the rate function. The Large Deviations lower bound is established in section 5 and the Large Deviations upper bound in section 6. Section 7 treats the exit time from a domain, including the case of a characteristic boundary. Finally in section 8 we show how our results apply to the SIRS model (which requires an additional argument), and a Girsanov theorem is formulated in the Appendix.

\section{Set-up}

We consider a set $A \subset \mathbb{R}^{d}$ (whose properties we specify below) and define the grids

$$
\mathbb{Z}^{d, N}:=\left\{x \in \mathbb{R}^{d} \mid x_{i}=j / N \text { for some } j \in \mathbb{Z}\right\}, \quad A^{N}:=A \cap \mathbb{Z}^{d, N} .
$$

We rewrite the process defined by Equation (1) as

$$
\begin{aligned}
Z^{N, x}(t):=Z^{N}(t) & :=x+\frac{1}{N} \sum_{j=1}^{k} h_{j} P_{j}\left(\int_{0}^{t} N \beta_{j}\left(Z^{N}(s)\right) d s\right) \\
& =x+\int_{0}^{t} b\left(Z^{N}(s)\right) d s+\frac{1}{N} \sum_{j} h_{j} M_{j}\left(\int_{0}^{t} N \beta_{j}\left(Z^{N}(s)\right) d s\right),
\end{aligned}
$$

where the $M_{j}(t)=P_{j}(t)-t$ are the compensated Poisson processes corresponding to the $P_{j}(j=1, \ldots, k)$.

Let us shortly comment on this definition. In the models we have in mind, the components of $Z^{N}$ usually denote the proportions of individuals in the respective compartments. It is hence plausible to demand that the starting point is in $\mathbb{Z}^{d, N}$. However, it is not sufficient to simply restrict our analysis to those starting points with $x \in A^{N}$ as this does obviously not imply $x \in A^{M}$ for all $M>N$. Note that $U^{N}(t)=N Z^{N}(t)$ would solve the SDE

$$
U^{N}(t)=N x+\sum_{j=1}^{k} h_{j} P_{j}\left(\int_{0}^{t} \beta_{j, N}\left(U^{N}(s)\right)\right),
$$

where $N \beta_{j}(x)=\beta_{j, N}(N x)$. Here the coefficients of the vector $U^{N}(t)$ are the numbers of individuals from the population in each compartment. The equation for $U^{N}(t)$ is really the original model, where all events of infection, recovery, loss of immunity, etc. are modeled. Dividing by $N$ leads to a process which has a law of large number limits as $N \rightarrow \infty$. The crucial assumption for this procedure to make sense is that $N^{-1} \beta_{j, N}(N x)$ does not depend upon $N$, which is typically the case in the epidemics models, see in particular Example 1.1 .

We first introduce the following notations. For $x \in A$ and $y \in \mathbb{R}^{d}$, let

$$
\begin{aligned}
V_{x} & :=\left\{\mu \in \mathbb{R}_{+}^{k} \mid \mu_{j}>0 \text { only if } \beta_{j}(x)>0\right\}, \\
V_{x, y} & :=\left\{\mu \in V_{x} \mid y=\sum_{j} \mu_{j} h_{j}\right\} .
\end{aligned}
$$


As $V_{x, y}$ is sometimes independent of $x$ or $V_{x, y}=\emptyset$, we also define for $y \in \mathbb{R}^{d}$,

$$
\tilde{V}_{y}:=\left\{\mu \in \mathbb{R}_{+}^{d} \mid y=\sum_{j} \mu_{j} h_{j}\right\}
$$

We define the cone spanned by a (finite) set of vectors $\left(v_{j}\right)_{j}\left(v_{j} \in \mathbb{R}^{d}\right.$ by

$$
\mathcal{C}\left(\left(v_{j}\right)_{j}\right):=\left\{v=\sum_{j} \alpha_{j} v_{j} \mid \alpha_{j} \geq 0\right\}
$$

Similarly, we define the cone generated by the jump directions $\left(h_{j}\right)_{j}$ at $x \in A$ by

$$
\mathcal{C}_{x}:=\mathcal{C}\left(\left(h_{j}\right)_{j: \beta_{j}(x)>0}\right)=\left\{v=\sum_{j: \beta_{j}(x)>0} \mu_{j} h_{j} \mid \mu_{j} \geq 0\right\} .
$$

Note that

$$
\mathcal{C}_{x}=\mathcal{C}=\left\{v=\sum_{j=1}^{k} \mu_{j} h_{j} \mid \mu_{j} \geq 0\right\}
$$

whenever $x \in \AA$, since $\beta_{j}(x)>0$ for all $1 \leq j \leq k$ if $x \in \AA$. Also, in part of this paper, we shall assume that the $\log \beta_{j}$ 's are bounded, which then means that (4) is true for all $x \in A$.

We define the following upper and lower bounds of the rates. Let $\rho>0$.

$$
\begin{aligned}
\bar{\beta} & :=\sup _{x \in A, j=1, \ldots, k} \beta_{j}(x) \in \overline{\mathbb{R}}_{+}, \\
\underline{\beta} & :=\inf _{x \in A, j=1, \ldots, k} \beta_{j}(x) \in \mathbb{R}_{+}, \\
\underline{\beta}(\rho) & :=\inf \left\{\beta_{j}(x) \mid j=1, \ldots, k, x \in A \text { and }|x-z| \geq \rho \forall z \in A \text { with } \beta_{j}(z)=0\right\} \in \mathbb{R}_{+}, \\
\bar{h} & :=\sup _{j=1, \ldots, k}\left|h_{j}\right| \in \mathbb{R}_{+} .
\end{aligned}
$$

\subsection{The Legendre-Fenchel transform and the rate function}

We define the following transforms. For $x \in A, y \in \mathbb{R}^{d}$, let

$$
\ell(x, \mu):=\sum_{j}\left\{\beta_{j}(x)-\mu_{j}+\mu_{j} \log \left(\frac{\mu_{j}}{\beta_{j}(x)}\right)\right\},
$$

with the convention $0 \log (0 / \alpha)=0$ for all $\alpha \in \mathbb{R}$, and

$$
\bar{L}(x, y):= \begin{cases}\inf _{\mu \in V_{x, y}} \ell(x, \mu) & \text { if } V_{x, y} \neq \emptyset \\ +\infty & \text { otherwise. }\end{cases}
$$

Now let, for $x, y$ as above and $\theta \in \mathbb{R}^{d}$,

$$
\tilde{\ell}(x, y, \theta)=\langle\theta, y\rangle-\sum_{j} \beta_{j}(x)\left(\mathrm{e}^{\left\langle\theta, h_{j}\right\rangle}-1\right),
$$

and define

$$
\underline{L}(x, y):=\sup _{\theta \in \mathbb{R}^{d}} \tilde{\ell}(x, y, \theta)
$$


Remark 2.1. For $\mu \in \tilde{V}_{y} \backslash V_{x, y}$, we have $\ell(x, \mu)=\infty$ and hence

$$
L(x, y)=\inf _{\mu \in \tilde{V}_{y}} \ell(x, \mu) .
$$

We first show

Lemma 2.2. Let $x \in A, y \in \mathcal{C}_{x}, \theta \in \mathbb{R}^{d}$ and $\mu \in V_{x, y}$. Then

$$
\tilde{\ell}(x, y, \theta) \leq \ell(x, \mu)
$$

in particular

$$
\underline{L}(x, y) \leq \bar{L}(x, y) .
$$

Proof. The result is obvious if $V_{x, y}=\emptyset$. If not, for $\mu \in V_{x, y}$, with

$$
\begin{aligned}
& f_{j}(z)=\mu_{j} z-\beta_{j}(x)\left(e^{z}-1\right), \\
\tilde{\ell}(x, y, \theta)= & \sum_{j} \mu_{j}\left\langle\theta, h_{j}\right\rangle-\beta_{j}(x)\left(\exp \left(\left\langle\theta, h_{j}\right\rangle\right)-1\right) \\
= & \sum_{j} f_{j}\left(\left\langle\theta, h_{j}\right\rangle\right) \\
\leq & \sum_{j} f_{j}\left(\log \mu_{j} / \beta_{j}(x)\right) \\
= & \ell(x, \mu),
\end{aligned}
$$

since $f_{j}$ achieves its maximum at $z=\log \left[\mu_{j} / \beta_{j}(x)\right]$.

We will show below that under appropriate assumptions, $\underline{L}(x, y)=\bar{L}(x, y)$, and we shall write $L(x, y)$ for the common value of those two quantities.

For any $T>0$, we define

$$
\begin{aligned}
& C([0, T] ; A):=\{\phi:[0, T] \rightarrow A \mid \phi \text { continuous }\} \\
& D([0, T] ; A):=\{\phi:[0, T] \rightarrow A \mid \phi \text { càdlàg }\} .
\end{aligned}
$$

On $C([0, T] ; A)$ (or $D([0, T] ; A)), \mathrm{d}_{C}$ denotes the metric corresponding to the supremumnorm, denoted by $\|\cdot\|$. Whenever the context is clear, we write $\mathrm{d}:=\mathrm{d}_{C}$. On $D([0, T] ; A)$ we denote by $\mathrm{d}_{D}$ the metric given, e.g., in Billingsley [1999], Sections 12.1 and 12.2 which defines the Skorohod topology in such a way that the resulting space is Polish. The resulting metric spaces are denoted by $C\left([0, T] ; A ; \mathrm{d}_{C}\right), D\left([0, T] ; A ; \mathrm{d}_{C}\right)$ and $D\left([0, T] ; A ; \mathrm{d}_{D}\right)$, respectively (where the metrics are omitted, whenever they are clear from the context).

We now introduce a candidate $I$ for the rate function. For $\phi:[0, T] \rightarrow A$, let

$$
I_{T}(\phi):= \begin{cases}\int_{0}^{T} L\left(\phi(t), \phi^{\prime}(t)\right) d t & \text { if } \phi \text { is absolutely continuous } \\ \infty & \text { otherwise. }\end{cases}
$$

For $x \in A$ and $\phi:[0, T] \rightarrow A$, let

$$
I_{T, x}(\phi):= \begin{cases}I_{T}(\phi) & \text { if } \phi(0)=x \\ \infty & \text { otherwise }\end{cases}
$$




\subsection{Assumptions on the process $Z^{N}$}

We do not assume that the rates are bounded away form zero (as in Shwartz and Weiss [1995]) and allow for them to vanish near the boundary (cf. the discussion in the introduction). Shwartz and Weiss [2005] allow for vanishing rates. We generalize these assumptions as we outline below. The difference is essentially Assumption 2.3 (C) below.

Assumption 2.3. (A) Assumptions on the set $A$.

(A1) The set $A$ is compact and $A=\bar{\AA}$. Furthermore, there exists a constant $\lambda_{0}>0$ such that for all $N \in \mathbb{N}, z \in A^{N}$ and $j=1, \ldots, k$ with $\beta_{j}(z)>0$,

$$
z+\frac{h_{j}}{N} \in A^{N} \quad \text { and } \quad|\tilde{z}-z| \geq \frac{\lambda_{0}}{N} \text { for all } \tilde{z} \text { with } \beta_{j}(\tilde{z})=0 \text { 2 }
$$

(A2) There exist open balls $B_{i}=B\left(x_{i}, r_{i}\right) \sqrt[3]{3}, i=1, \ldots, I_{1}, \ldots, I\left(0<I_{1}<I\right)$ such that

$$
x_{i} \in \partial A \text { for } i \leq I_{1} \quad \text { and } \quad x_{i} \in \AA \text { for } i>I_{1}
$$

and

$$
A \subset \bigcup_{i \leq I} B_{i}, \quad \partial A \subset \bigcup_{i \leq I_{1}} B_{i} \quad \text { and } \quad B_{i} \cap \partial A=\emptyset \text { for } i>I_{1} .
$$

(A3) There exist (universal) constants $\lambda_{1}, \lambda_{2}>0$ and vectors $v_{i}$ ( $i \leq I_{1}$, w.l.o.g., we assume $0<\left|v_{i}\right| \leq 1$; for notational reasons, we set $v_{i}=0$ for $i>I_{1}$ ) such that for all $x \in B_{i} \cap A$,

$$
B\left(x+t v_{i}, \lambda_{1} t\right) \subset A \quad \text { for all } t \in\left(0, \lambda_{2}\right) .
$$

and $\operatorname{dist}\left(x+t v_{i}, \partial A\right)$ is increasing for $t \in\left(0, \lambda_{2}\right)$.

(A4) There exists a Lipschitz continuous mapping $\psi_{A}: \mathbb{R}^{d} \rightarrow A$ such that $\psi_{A}(x)=x$ whenever $x \in A$.

(B) Assumptions on the rates $\beta_{j}$.

(B1) The rates $\beta_{j}: A \rightarrow \mathbb{R}_{+}$are Lipschitz continuous.

(B2) For $x \in \AA, j=1, \ldots, k, \beta_{j}(x)>0$ and $\mathcal{C}\left(\left(h_{j}\right)_{j}\right)=\mathbb{R}^{d}$.

(B3) For all $x \in \partial A$ there exists a constant $\lambda_{3}=\lambda_{3}(x)>0$ such that

$$
y \in \mathcal{C}_{x},|y| \leq \lambda_{3} \Rightarrow x+y \in A \text {. }
$$

(B4) There exists a (universal) constant $\lambda_{4}>0$ such that for all $i \leq I_{1}, x \in B_{i} \cap A$ and

$$
\begin{gathered}
v \in \mathcal{C}_{1, i}:=\left\{\frac{\tilde{v}}{|\tilde{v}|} \mid \tilde{v}=v_{i}+w \text { for } w \in \mathbb{R}^{d},|w| \leq \frac{\lambda_{1}}{3-\lambda_{1}}\right\}, \\
\beta_{j}(x)<\lambda_{4} \Rightarrow \beta_{j}(x+\cdot v) \text { is increasing in }\left(0, \lambda_{2}\right) .
\end{gathered}
$$

\footnotetext{
${ }^{2}$ This implies that for $z \in A^{N}$ with $\beta_{j}(z)>0$, we have $\beta_{j}(z) \geq \underline{\beta}\left(\lambda_{0} / N\right)$.

${ }^{3}$ Here (and later) $B(x, r)$ denotes the open ball around $x$ with radius $r$.
} 
(C) There exists an $\eta_{0}>0$ such that for all $N \in \mathbb{N}, \epsilon>0$ there exists a constant $\delta(N, \epsilon)>0$ (decreasing in $N$ and in $\epsilon$ ) such that for all $i \leq I_{1}, x \in B_{i}$ there exists $a \mu^{i}=\mu^{i}(x) \in \tilde{V}_{v_{i}}$ and

$$
\mathbb{P}\left[\sup _{t \in\left[0, \eta_{0}\right]}\left|\tilde{Z}^{N, x}(t)-\phi^{x}(t)\right| \geq \epsilon\right] \leq \delta(N, \epsilon)
$$

where $\tilde{Z}^{N, x}$ denotes the solution of (3) if the rates $\beta_{j}$ are replaced by the rates $\tilde{\mu}_{j}^{i}$ for

$$
\tilde{\mu}_{j}^{i}(z):= \begin{cases}\mu_{j}^{i} & \text { if } z+\epsilon h_{j} \in A \text { for all } \epsilon \text { small enough } \\ 0 & \text { else }\end{cases}
$$

and $\phi^{x}=x+t v_{i}$ as before 4

Furthermore, there exists a constant $\alpha \in(0,1 / 2)$ and a sequence $\epsilon_{N}$ such that

$$
\epsilon_{N}<\frac{1}{N^{\alpha}} \text { and } \quad \frac{\delta\left(N, \epsilon_{N}\right)}{\epsilon_{N}} \rightarrow 0 \text { as } N \rightarrow \infty .
$$

and

$$
\rho^{\alpha} \log \underline{\beta}(\rho) \rightarrow 0 \quad \text { as } \rho \rightarrow 0 .
$$

Let us comment on Assumption 2.3. Assumption (A) is essentially Assumption 2.1 of Shwartz and Weiss 2005]. We want to remark that Assumption 2.1 (iv) of Shwartz and Weiss [2005] is not included here as it is redundant (see Lemma 3.5 of Shwartz and Weiss [2005]; cf. also the discussion preceding Lemma 5.5). In the epidemiological models we want to consider, $A$ is a compact, convex $d$-polytope, i.e., $\partial A$ is composed by $d$-1-dimensional hyperplanes. For example for the SIRS model in Example 1.1,

$$
A=\left\{x \in \mathbb{R}^{2} \mid 0 \leq x_{1}+x_{2} \leq 1\right\} .
$$

In line with the Assumption (A1), let us note that we always want to choose the starting point $x$ of equation (11) to belong to $A^{N}$. If that would not be the case, then in our simplest models the solution $Z^{N, x}$ might exit the domain $A$. Choosing the starting point arbitrarily in $A$ would force us to let the rates $\beta_{j}$ depend upon $N$ (and vanish) near the boundary. Note that the coordinates of the vector $Z_{t}^{N, x}$ are proportions of the population in various compartments. The coordinates of the vector $N Z_{t}^{N, x}$ are integers, while those of the vectors $h_{j}$ belong to the set $\{-1,0,1\}$.

Note that in all situations we have in mind, both the set $A$ itself and its boundary can be covered by a finite number of balls. These balls can furthermore be chosen in such a way that those centered in the interior do not intersect with the boundary. For the SIRS model, we can for instance define the balls covering the boundary by $B(x, 3 /(4 m))$ for large $m \in \mathbb{N}$ and $x=(i / m, j / m)^{\top}$ for $i=0, \ldots, m, j=0$ or $i=0, j=0, \ldots, m$ or $i+j=m$. The vectors $v_{i}$ can be defined to be the inside normal vectors for those balls with $x \notin\left\{(0,0)^{\top},(1,0)^{\top},(0,1)^{\top}\right\}$. For the remaining three balls, we define $v_{i}$ by the normalizations of

$$
(1 / 2,1 / 2)^{\top}, \quad(-1 / 2,1 / 4)^{\top} \quad \text { respectively }(1 / 2,-1 / 2)^{\top} .
$$

\footnotetext{
${ }^{4}$ We do not necessarily have $\mu^{i} \in V_{x, v_{i}}$ for all $x \in B_{i}$; this might not be the case if $x \in \partial A$. In such a case $V_{x, v_{i}}=\emptyset$ is possible, cf. the discussion about $x=(1,0)^{\top}$ for the SIRS model below.
} 
In general, the constant $\lambda_{1}$ can be interpreted to be given via the "angle" of the vector $v_{i}$ to the boundary. We have $\lambda_{1} \leq 1$. It is straightforward that Assumption (A) is satisfied for the SIRS model. We also note that Assumption (A) is not very restrictive, see Shwartz and Weiss [2005] Lemma 2.1. In particular, every convex, compact set with non-empty interior satisfies the assumption.

Most of Assumption (B) is taken from Assumption 2.2 of Shwartz and Weiss [2005]. We outline the difference below. Assumption (B1) is quite standard and ensures in particular that the ODE (2) admits a unique solution. For the compartmental epidemiological models we consider, the rates are usually polynomials and hence this assumption is satisfied. Assumption (B2) implies that within $\AA$ it is possible to move into all directions. Only by approaching the boundary the rates are allowed to vanish. (B3) implies that at least locally the convex cone $x+\mathcal{C}_{x}$ is included in $A$. In particular, it is not possible to exit the set $A$ from its boundary. Assumption (B4) differs slightly from the corresponding assumption in Shwartz and Weiss [2005]. While in Shwartz and Weiss [2005], it is implied that close to the boundary, "small" rates are increasing while following the vector $v_{i}$, we assume this for a set of vector in a "cone" around $v_{i}$. We note that for $i \leq I_{1}, x \in B_{i}$, $v \in \mathcal{C}_{1, i}$, we have (cf. Assumption (A3))

$$
\operatorname{dist}(x+t v, \partial A) \geq \operatorname{dist}\left(x+t v_{i}, \partial A\right)-t \frac{\lambda_{1}}{3-\lambda_{1}} \geq t \lambda_{1}\left(\frac{2-\lambda_{1}}{3-\lambda_{1}}\right) .
$$

It is easily seen that this assumption is satisfied for the SIRS model. In addition to this, Shwartz and Weiss [2005] also require that (cf. the meaning of $\lambda_{4}$ in Assumption (B4))

$$
v_{i} \in \mathcal{C}\left(\left\{h_{j} \mid \inf _{x \in B_{i}} \beta_{j}(x)>\lambda_{4}\right\}\right) .
$$

In order to apply the theory to epidemiological models, we have to remove this assumption. To see this, consider the SIRS model and the point $x=(1,0)^{\top}$ with corresponding ball $B$ containing it. We readily observe that a vector $v$ pointing "inside" $A$ (as required by Assumption (A3)) which is generated by only those $h_{j}$ whose corresponding rates are bounded away from zero in $B$ does not exist. We hence replace this assumption by Assumption (C), which follows from (10). Indeed, if Assumption (10) holds, the $\mu^{i}$ representing $v_{i}$ can be chosen in such a way that the directions corresponding to components $\mu_{j}^{i}>0$ do not point outside $A$ in $B_{i}$. Hence, $\tilde{\mu}^{i} \equiv \mu^{i}$ (as long as the process is in $B_{i}$ ) and the LLN Theorem 3.1 can be applied. In general, Theorem 3.1 cannot be applied as the rates $\tilde{\mu}^{i}$ can be discontinuous. Note that the assumption can only fail if $x \in \partial A$. Else, the process is equal to the process with constant rates $\mu^{i}$ on the set

$$
\left\{\sup _{t \in\left[0, \eta_{0}\right]}\left|\tilde{Z}^{N, x}(t)-\phi^{x}(t)\right|<\epsilon\right\}
$$

for all small enough $\epsilon>0$ and Theorem 3.1 is applicable. We note that Assumption (C) implies that

$$
\delta(N, \epsilon) \rightarrow 0 \text { as } N \rightarrow \infty \text { for all } \epsilon>0 .
$$

Moreover, as $\delta(N, \cdot)$ is decreasing, we can choose $\epsilon_{N}$ in such a way that

$$
\epsilon_{N}=\frac{1}{N^{\alpha}} \quad \text { for some } \alpha \in(0,1) \text {. }
$$


We remark here (and further discuss this important issue below) that Assumption $2.3(\mathrm{C})$ may well fail to be satisfied. To this end, we consider the SIRS model an $x \in A$ with $x_{1}=0$. We hence have $\beta_{2}(x)=0$ and hence the process $Z^{N, x}$ cannot enter the interior of $A$. Therefore, we have

$$
\mathbb{P}\left[\sup _{t \in\left[0, \eta_{0}\right]}\left|Z^{N, x}(t)-\phi^{x}(t)\right| \geq \epsilon\right]=1
$$

for $\epsilon$ small enough. Assumption (C) can hence be considered as a means to ensure that the process can enter the interior of $A$ from every point on the boundary. (9) implies that

$$
\int_{0}^{\eta}|\log \underline{\beta}(\rho)| d \rho \rightarrow 0 \quad \text { as } \eta \rightarrow 0
$$

since $\rho^{\alpha / 2}|\log \underline{\beta}(\rho)| \leq C$ for appropriate $C$ and hence $|\log \underline{\beta}(\rho)| \leq C / \rho^{\alpha / 2}$ is integrable, and hence in particular that the rate $I(\phi)$ of linear functions $\phi$ is finite, as it is shown in Lemma 5.7 below.

It remains to show that Assumption 2.3 (C) is satisfied for the SIRS model. This is accomplished in section 8 .

Exploiting (91), it is easy to prove

Lemma 2.4. Under the Assumption[2.3 (C), for all $i \leq I, x \in A \cap B_{i}$, let $\phi^{x}(t):=x+t v_{i}$. For all $\epsilon>0$, there exists an $\eta>0$ (independent of $i, x$ ) such that for all $i \leq I$ and all $x \in A \cap B_{i}$,

$$
I_{\eta, x}\left(\phi^{x}\right)<\epsilon .
$$

Note that for $i>I_{1}$, we have $\phi^{x}(t)=x$ for all $t$.

\section{Law of large numbers}

We first prove the law of large numbers by Kurtz [1978] with the rate of convergence given as in Shwartz and Weiss [1995] Theorem 5.3.

Theorem 3.1. Let $Z^{N . x}$ and $Y^{x}$ be given as in Equation (3) and (2) respectively, and assume that the rates $\beta_{j}$ are bounded and Lipschitz continuous. Then there exist constants $\tilde{C}_{1}=\tilde{C}_{1}(T)>0$ (independent of $\epsilon$ ) and $\tilde{C}_{2}(\epsilon)=\tilde{C}_{2}(T, \epsilon)>0$ with $\tilde{C}_{2}(\epsilon)=O\left(\epsilon^{2}\right)$ as $\epsilon \rightarrow 0$ such that

$$
\mathbb{P}\left[\sup _{t \in[0, T]}\left|Z^{N, x}(t)-Y^{x}(t)\right| \geq \epsilon\right] \leq \tilde{C}_{1} \exp \left(-N \tilde{C}_{2}(\epsilon)\right) .
$$

$C_{1}$ and $C_{2}$ can be chosen independently of $x$.

Before we prove Theorem 3.1, we require some auxiliary results. We first have

Lemma 3.2. Let $T>0$. Suppose that $f: D([0, T] ; A) \times \mathbb{R} \rightarrow \mathbb{R}$ and $G: D([0, T] ; A) \times$ $\mathbb{R} \times \mathbb{R} \rightarrow \mathbb{R}$ are such that for all $\rho>0$,

$$
M(t):=\exp \left(\rho f\left(Z^{N, x}, t\right)-G\left(Z^{N, x}, t, \rho\right)\right)
$$

is a right-continuous martingale with mean one. Suppose furthermore that $R: \mathbb{R} \times \mathbb{R} \rightarrow \mathbb{R}$ is increasing in the first argument and

$$
G(\phi, t, \rho) \leq R(t, \rho)
$$


for all $\phi \in D([0, T] ; A)$ and $\rho>0$. Then for all $\epsilon>0$

$$
\mathbb{P}\left[\sup _{t \in[0, T]} f\left(Z^{N, x}, t\right) \geq \epsilon\right] \leq \inf _{\rho>0} \exp (R(T, \rho)-\rho \epsilon) .
$$

Proof. Fix $\rho>0$. Then by the assumptions of the lemma,

$$
\begin{aligned}
\mathbb{P}\left[\sup _{t \in[0, T]} f\left(Z^{N, x}, t\right) \geq \epsilon\right] & =\mathbb{P}\left[\sup _{t \in[0, T]} \exp \left(\rho f\left(Z^{N, x}, t\right)\right) \geq \exp (\rho \epsilon)\right] \\
& \leq \mathbb{P}\left[\sup _{t \in[0, T]} \exp \left(\rho f\left(Z^{N, x}, t\right)-G\left(Z^{N, x}, t, \rho\right)\right) \geq \exp (\rho \epsilon-R(T, \rho))\right] \\
& \leq \exp (R(T, \rho)-\rho \epsilon)
\end{aligned}
$$

where the last inequality is Doob's martingale inequality, see, e.g. Theorem II.1.7 in Revuz and Yor [2005].

The next result is an easy exercise which we leave to the reader.

Lemma 3.3. Let $Y$ be a d-dimensional random vector. Suppose that there exist numbers $a>0$ and $\delta>0$ such that for all $\theta \in \mathbb{R}^{d}$ with $|\theta|=1$

$$
\mathbb{P}[\langle\theta, Y\rangle \geq a] \leq \delta .
$$

Then

$$
\mathbb{P}[|Y| \geq a \sqrt{d}] \leq 2 d \delta
$$

The main step towards the proof of Theorem 3.1 is the following Lemma

Lemma 3.4. Assume that $\beta_{j}(j=1, \ldots, k)$ is bounded and that $Y^{x}$ is a solution of (2). Then for all $\theta \in \mathbb{R}^{d}$ with $|\theta|=1$ and all $T>0$, there is a function $\tilde{C}: \mathbb{R}_{+} \rightarrow \mathbb{R}_{+}$ (independent of $x$ ) such that

$\mathbb{P}\left[\sup _{t \in[0, T]}\left\{\left\langle Z^{N, x}(t)-Y^{x}(t), \theta\right\rangle-\int_{0}^{t} \sum_{j=1}^{k}\left(\beta_{j}\left(Z^{N, x}(s)\right)-\beta_{j}\left(Y^{x}(s)\right)\right)\left\langle h_{j}, \theta\right\rangle d s\right\} \geq \epsilon\right] \leq \exp (-N \tilde{C}(\epsilon))$,

and moreover

$$
0<\lim _{\epsilon \rightarrow 0} \tilde{C}(\epsilon) / \epsilon^{2}<\infty, \quad \text { and } \quad \lim _{\epsilon \rightarrow \infty} \tilde{C}(\epsilon) / \epsilon=\infty
$$

Proof. Let

$$
\begin{aligned}
\mathcal{N}_{t}^{\theta} & =\left\langle Z^{N, x}(t)-Y^{x}(t), \theta\right\rangle-\int_{0}^{t} \sum_{j=1}^{k}\left(\beta_{j}\left(Z^{N, x}(s)\right)-\beta_{j}\left(Y^{x}(s)\right)\right)\left\langle\theta, h_{j}\right\rangle d s \\
& =\frac{1}{N} \sum_{j=1}^{k}\left\langle h_{j}, \theta\right\rangle M_{j}\left(N \int_{0}^{t} \beta_{j}\left(Z_{s}^{N, x}\right) d s\right) .
\end{aligned}
$$

We want to use Lemma [3.2, with $f\left(Z^{N, x}, t\right)=\mathcal{N}_{t}^{\theta}$. It is not hard to check that if we define

$$
G\left(Z^{N, x}, t, \rho\right)=N \sum_{j=1}^{k}\left(e^{\frac{\rho}{N}\left\langle h_{j}, \theta\right\rangle}-1-\frac{\rho}{N}\left\langle h_{j}, \theta\right\rangle\right) \int_{0}^{t} \beta_{j}\left(Z^{N, x}(s)\right) d s
$$


we have that

$$
M(t)=\exp \left(\rho f\left(Z^{N, x}, t\right)-G\left(Z^{N, x}, t, \rho\right)\right)
$$

is a martingale. Hence from Lemma [3.2, with $a=\rho / N$,

$$
\mathbb{P}\left(\sup _{0 \leq t \leq T} \mathcal{N}_{t}^{\theta}>\epsilon\right) \leq \min _{a>0} \exp \left(N \bar{\beta} T\left[\sum_{j=1}^{k}\left\{e^{a\left\langle h_{j}, \theta\right\rangle}-1-a\left\langle h_{j}, \theta\right\rangle\right\}-a \epsilon\right]\right) .
$$

The main inequality of the Lemma is established, with

$$
\tilde{C}(\epsilon)=\bar{\beta} T \max _{a>0}\left[a \epsilon-\sum_{j=1}^{k}\left\{e^{a\left\langle h_{j}, \theta\right\rangle}-1-a\left\langle h_{j}, \theta\right\rangle\right\}\right] .
$$

It is not hard to show that as $\epsilon \rightarrow 0$,

$$
\frac{\tilde{C}(\epsilon)}{\epsilon^{2}} \rightarrow \frac{\bar{\beta} T}{2 \sum_{j=1}^{k}\left\langle h_{j}, \theta\right\rangle^{2}} .
$$

Consider now the case where $\epsilon$ is large. If $\left\langle h_{j}, \theta\right\rangle \leq 0$ for $1 \leq j \leq k$, then for $\epsilon>$ $-\sum_{j}\left\langle h_{j}, \theta\right\rangle, \tilde{C}(\epsilon)=+\infty$, which means that a certain event has probability zero. Now consider the more interesting case where $\left\langle h_{j}, \theta\right\rangle>0$ for at least one $1 \leq j \leq k$. If we choose $a_{\epsilon}$ such that

$$
\sum_{j=1}^{k}\left\{e^{a_{\epsilon}\left\langle h_{j}, \theta\right\rangle}-1-a_{\epsilon}\left\langle h_{j}, \theta\right\rangle\right\}=\epsilon,
$$

then $a_{\epsilon} \rightarrow \infty$ as $\epsilon \rightarrow \infty$, while $\tilde{C}(\epsilon) \geq \epsilon\left(a_{\epsilon}-1\right)$, which completes the proof of the Lemma.

Proof of Theorem 3.1. We deduce from Lemma 3.4 and a variant of Lemma 3.3 that

$$
\mathbb{P}\left(\sup _{0 \leq t \leq T} \frac{1}{N}\left|\sum_{j=1}^{k} h_{j} M_{j}\left(N \int_{0}^{t} \beta_{j}\left(Z_{s}^{N, x}\right) d s\right)\right|>\epsilon\right) \leq 2 d e^{-N \tilde{C}^{\prime}(\epsilon)}
$$

where $\tilde{C}^{\prime}(\epsilon)=\tilde{C}(\epsilon / \sqrt{d})$. In view of the Lipschitz property of $b$, we have

$$
\begin{gathered}
Z_{t}^{N, x}-Y_{t}^{x}=\int_{0}^{t}\left[b\left(Z_{s}^{N, x}\right)-b\left(Y_{s}^{x}\right)\right] d s+\frac{1}{N} \sum_{j=1}^{k} h_{j} M_{j}\left(N \int_{0}^{t} \beta_{j}\left(Z_{s}^{N, x}\right) d s\right), \\
\sup _{0 \leq s \leq t}\left|Z_{s}^{N, x}-Y_{s}^{x}\right| \leq K \int_{0}^{t} \sup _{0 \leq r \leq s}\left|Z_{r}^{N, x}-Y_{r}^{x}\right| d s+\sup _{0 \leq s \leq t} \frac{1}{N}\left|\sum_{j=1}^{k} h_{j} M_{j}\left(N \int_{0}^{s} \beta_{j}\left(Z_{r}^{N, x}\right) d r\right)\right| .
\end{gathered}
$$

The result now follows from (13) and Gronwall's Lemma.

We can deduce from Theorem 3.1 . 
Corollary 3.5. Let $M$ be a compensated standard Poisson process. Then there exist constants $C_{1}=C_{1}(T)>0$ (independent of $\epsilon$ ) and $C_{2}(\epsilon)=C_{2}(T, \epsilon)>0$ with $C_{2}(\epsilon)=O\left(\epsilon^{2}\right)$ as $\epsilon \rightarrow 0$ such that

$$
\mathbb{P}\left[\sup _{t \in[0, T]} \frac{|M(t N)|}{N} \geq \epsilon\right] \leq C_{1} \exp \left(-N C_{2}(\epsilon)\right) .
$$

$C_{1}$ and $C_{2}$ can be chosen independently of $x$.

Proof. We apply Theorem 3.1 to $d=k=1, \beta_{1}(x) \equiv 1$ and $h_{1}=1$. Hence,

$$
\left|Z^{N}(t)-Y(t)\right|=\frac{|M(t N)|}{N} .
$$

The result follows directly.

We shall need below the

Lemma 3.6. Let $\beta_{j}(j=1, \ldots, k)$ be bounded. Then there exist positive constants $\tilde{C}_{1}$ and $\tilde{C}_{2}$ independent of $x$ such that for all $0 \leq s<t \leq T$ and for all $\epsilon>0$,

$$
\mathbb{P}\left[\sup _{r \in[s, t]}\left|Z^{N, x}(r)-Z^{N, x}(s)\right| \geq \epsilon\right] \leq \exp \left(-N \epsilon \tilde{C}_{1} \log \left(\frac{\epsilon \tilde{C}_{2}}{t-s}\right)\right) .
$$

Proof. Let $\xi_{s, t}^{N}$ denote the number of jumps of the process $Z^{N, x}$ on the time interval $[s, t]$. It is plain that

$$
\left\{\sup _{r \in[s, t]}\left|Z^{N, x}(r)-Z^{N, x}(s)\right| \geq \epsilon\right\} \subset\left\{\xi_{s, t}^{N} \geq C N \epsilon\right\},
$$

for some universal constant $C>0$. Now $\xi_{s, t}^{N}$ is stochastically dominated by a Poisson random variable with parameter $C^{\prime} N(t-s)$, for some other constant $C^{\prime}>0$. Now let $\Theta$ be a Poisson r.v. with parameter $\lambda$. For any $a, b>0$,

$$
\begin{aligned}
\mathbb{P}(\Theta>b) & =\mathbb{P}\left(e^{a \Theta}>e^{a b}\right) \\
& \leq \exp \left(\lambda\left(e^{a}-1\right)-a b\right),
\end{aligned}
$$

which, with the optimal choice $a=\log (b / \lambda)$, reads

$$
\mathbb{P}(\Theta>b) \leq \exp (b-\lambda-b \log (b / \lambda)) \leq \exp \left(-b \log \left(\frac{b}{e \lambda}\right)\right) .
$$

The result follows by applying this inequality with $\lambda=C^{\prime} N(t-s)$, and $b=C N \epsilon$.

\section{Properties of the rate function}

\subsection{Properties of the Legendre Fenchel transform}

In this subsection we assume that the $\beta_{j}$ 's are bounded and continuous. We recall that $\ell$, $\bar{L}, \tilde{\ell}$ and $\underline{L}$ have been defined in section 2.1 , and start with

Lemma 4.1. 1. For all $x \in A, \underline{L}(x, \cdot): \mathcal{C}_{x} \rightarrow \mathbb{R}_{+}$is convex and lower semicontinuous. 
2. For all $y \in \mathbb{R}^{d}$,

$$
\underline{L}(x, y) \geq \underline{L}\left(x, \sum_{j} \beta_{j}(x) h_{j}\right)=0
$$

with strict inequality if $y \neq \sum_{j} \beta_{j}(x) h_{j}$.

Proof. 1. We readily observe that $\tilde{\ell}(x, \cdot, \theta)$ is linear and hence convex. As the supremum of these functions, the function $\underline{L}(x, \cdot)$ is convex.

Lower semicontinuity follows as $\underline{L}(x, \cdot)$ is the supremum of a family of continuous functions.

2. Let first $y=\sum_{j} \beta_{j}(x) h_{j}$. We have

$$
\begin{aligned}
\underline{L}(x, y) & =\sup _{\theta}\left\{\sum_{j} \beta_{j}(x)\left\langle h_{j}, \theta\right\rangle-\sum_{j} \beta_{j}(x)\left(\exp \left\langle h_{j}, \theta\right\rangle-1\right)\right\} \\
& =\sup _{\theta}\left\{\sum_{j} \beta_{j}(x)\left(\left\langle h_{j}, \theta\right\rangle-\exp \left\langle h_{j}, \theta\right\rangle+1\right)\right\} \\
& =0
\end{aligned}
$$

as $\beta_{j}(x) \geq 0$ and $e^{z} \geq z+1$ for all $z \in \mathbb{R}$ with equality for $z=0$.

Let now $y$ be such that $\underline{L}(x, y)=0$. This implies

$$
\langle y, \theta\rangle-\sum_{j} \beta_{j}(x)\left(\exp \left\langle h_{j}, \theta\right\rangle-1\right) \leq 0 \quad \text { for all } \theta \in \mathbb{R}^{d},
$$

in particular for $\theta=\epsilon e_{i}$ (where $e_{i}$ is the $i^{\text {th }}$ unit-vector and $\epsilon>0$; in the following $h_{j}^{i}$ is the $i^{\text {th }}$ component of $h_{j}$ ),

$$
\epsilon y_{i} \leq \sum_{j} \beta_{j}(x)\left(\exp \left(\epsilon h_{j}^{i}\right)-1\right)
$$

Dividing by $\epsilon$ and letting $\epsilon \rightarrow 0$, we deduce that

$$
y_{i} \leq \sum_{j} \beta_{j}(x) h_{j}^{i}
$$

For $\theta=-\epsilon e_{i}$ the converse inequality follows accordingly.

Remark 4.2. The function $\underline{L}(x, \cdot)$ is even strictly convex, see Corollary 4.10 below.

Lemma 4.3. Assume that $\beta_{j}(j=1, \ldots, k)$ is bounded. Then, there exist constants $C_{1}$ and $B_{1}$ such that for all $|y| \geq B_{1}, x \in \mathbb{R}^{d}$,

$$
\underline{L}(x, y) \geq C_{1}|y| \log (|y|) .
$$

Proof. Let

$$
\theta:=y \frac{\log |y|}{\bar{h}|y|}
$$

hence provided $|y| \geq 1$,

$$
\underline{L}(x, y) \geq \frac{|y| \log |y|}{\bar{h}}-k \bar{\beta}|y|
$$

which grows like $|y| \log |y|$ as $|y| \rightarrow \infty$. 
We now have

Lemma 4.4. There exists a constant $C_{2}$ such that for all $x \in A, y \in \mathcal{C}_{x}$, there exists a $\mu \in V_{x, y}$ with

$$
|\mu| \leq C_{2}|y|
$$

Proof. We first note that there are only finitely many convex cones $\mathcal{C}_{x}$ and we can hence restrict our attention to a fixed $x \in A$.

We proceed by contradiction. Assume that for all $n$ there exists a $y^{n} \in \mathcal{C}_{x}$ such that for all $\mu \in V_{x, y^{n}}$,

$$
|\mu| \geq n\left|y^{n}\right|
$$

We note that for any $y \in \mathcal{C}_{x}$, there exists a minimal representation $\mu \in V_{x, y}$ (in the sense that $\left.\tilde{\mu} \in V_{x, y} \Rightarrow \max _{j} \tilde{\mu}_{j} \geq \max _{j} \mu_{j}\right)$. Indeed, let $\left\{\mu^{n},: n \geq 1\right\} \subset V_{x, y}$ be such that, as $n \rightarrow \infty$,

$$
\max _{j} \mu_{j}^{n} \downarrow \inf _{\mu \in V_{x, y}}\left(\max _{j} \mu_{j}\right) .
$$

There exists a subsequence along which $\mu^{n} \rightarrow \mu \in \mathbb{R}_{+}^{d}$ as $n \rightarrow \infty$. If $\mu_{j}>0$, we have $\mu_{j}^{n}>0$ for $n$ large enough and hence $\beta_{j}(x)>0$. Hence $\mu \in V_{x, y}$ since moreover

$$
\sum_{j} \mu_{j} h_{j}=\lim _{n} \sum_{j} \mu_{j}^{n} h_{j}=y
$$

Given $y^{n}$, we denote this minimal representation by $\bar{\mu}^{n}$. We now define

$$
\tilde{y}^{n}:=\frac{y^{n}}{\left|\bar{\mu}^{n}\right|}, \quad \text { hence } \quad\left|\tilde{y}^{n}\right| \leq \frac{1}{n} .
$$

Furthermore, it is easy to see that minimal representations for the $\tilde{y}^{n}$ are given by

$$
\tilde{\mu}^{n}:=\frac{\bar{\mu}^{n}}{\left|\bar{\mu}^{n}\right|}, \quad \text { hence } \quad\left|\tilde{\mu}^{n}\right|=1 .
$$

Boundedness implies (after possibly the extraction of a subsequence) $\tilde{\mu}^{n} \rightarrow \tilde{\mu}$ with $|\tilde{\mu}|=1$. We let $n$ large enough such that for all $j$

$$
\tilde{\mu}_{j}^{n}>0 \Rightarrow \tilde{\mu}_{j}^{n}>\frac{\tilde{\mu}_{j}}{2}
$$

(note that for at least one $j, \tilde{\mu}_{j}>0$ ). We have

$$
0=\lim _{n} \tilde{y}^{n}=\lim _{n} \sum_{j} \tilde{\mu}_{j}^{n} h_{j}=\sum_{j} \tilde{\mu}_{j} h_{j}
$$

and therefore

$$
\tilde{y}^{n}=\sum_{j} \tilde{\mu}_{j}^{n} h_{j}=\sum_{j ; \tilde{\mu}_{j}>0} \underbrace{\left(\tilde{\mu}_{j}^{n}-\frac{\tilde{\mu}_{j}}{2}\right)}_{=: \hat{\mu}_{j}^{n}>0} h_{j}+\sum_{j ; \tilde{\mu}_{j}=0} \underbrace{\tilde{\mu}_{j}^{n}}_{=: \hat{\mu}_{j}^{n}} h_{j},
$$

a contradiction to the minimality of the $\tilde{\mu}_{j}^{n}$.

We require the following result 
Lemma 4.5. Let $x \in A$.

1. $\ell(x, \mu) \geq 0$ for $\mu \in V_{x}$ and $\ell(x, \cdot): V_{x} \longrightarrow \mathbb{R}_{+}$is strictly convex and has compact level sets $\left\{\mu \in V_{x} \mid \ell(x, \mu) \leq \alpha\right\}$.

2. Let $y \in \mathcal{C}_{x}$. Then there exists a unique $\mu^{*}=\mu^{*}(y)$ such that

$$
\ell\left(x, \mu^{*}\right)=\inf _{\mu \in V_{x, y}} \ell(x, \mu)
$$

3. There exist constants $C_{3}, C_{4}, C_{5}, B_{2}>0$ (which depend only upon $\sup _{x \in A} \max _{i \leq j \leq k} \beta_{j}(x)$ ), such that

$$
\begin{aligned}
& \left|\mu^{*}(y)\right| \leq C_{3}|y| \quad \text { if }|y|>B_{2} \\
& \left|\mu^{*}(y)\right| \leq C_{4} \quad \text { if }|y| \leq B_{2} \\
& \left|\mu^{*}(y)\right| \geq C_{5}|y| \quad \text { for all } y
\end{aligned}
$$

4. $\bar{L}(x, \cdot), \mu^{*}: \mathcal{C}_{x} \rightarrow \mathbb{R}_{+}$are continuous.

Proof. 1. We define the function $f(z)=1-z+z \log z$ for $z \geq 0$ and note that for $\mu \in V_{x}$,

$$
\ell(x, \mu)=\sum_{j, \beta_{j}(x)>0} \beta_{j}(x) f\left(\frac{\mu_{j}}{\beta_{j}(x)}\right) .
$$

We readily observe (by differentiation) that $f \geq 0$ and that $f$ is strictly convex. Thus the first two assertions follow.

As $V_{x}$ is closed and $\ell(x, \cdot)$ is continuous, the level sets are closed. Compactness follows form the fact that $\lim _{x \rightarrow \infty} f(x)=\infty$.

2. Existence of a minimizer follows from the fact that $V_{x, y}$ is closed. Uniqueness follows from the strict convexity of $\ell(x, \cdot)$.

3. By the definition of $\ell$, there exists a $B_{2}=B_{2}(\bar{\beta}(x))>0$ and $C=C(\bar{\beta}(x))>0$ such that for $y \in \mathcal{C}_{x}$ with $|y| \geq B_{2}$ (and appropriate $\mu \in V_{x, y}$ according to Lemma 4.4),

$$
\ell\left(x, \mu^{*}(y)\right) \leq \ell(x, \mu) \leq C|y| \log |y| .
$$

On the other hand, assume that for all $n$ there exists an $y^{n} \in \mathcal{C}_{x}$ with $\left|y^{n}\right| \geq B_{2}$ such that

$$
\left|\mu^{*}\left(y^{n}\right)\right| \geq n\left|y^{n}\right|
$$

This implies for an appropriate constant $\tilde{C}$ and $n$ large enough

$$
\ell\left(x, \mu^{*}\left(y^{n}\right)\right) \geq n \tilde{C}|y| \log |y|,
$$

a contradiction. Hence Inequality (14) follows.

Assume now that for all $n$ there exists an $y^{n} \in \mathcal{C}_{x}$ with $\left|y^{n}\right| \leq B_{2}$,

$$
\left|\mu^{*}\left(y^{n}\right)\right| \geq n, \quad \text { hence } \lim _{n \rightarrow \infty} \ell\left(x, \mu^{*}\left(y^{n}\right)\right) \rightarrow \infty \text {. }
$$


However, Lemma 4.4 implies that there exists an $\mu^{n} \in V_{x, y^{n}}$ and a constant $C=$ $C\left(\bar{\beta}(x), B_{2}\right)$ independent of $n$ with

$$
\ell\left(x, \mu^{n}\right) \leq C,
$$

a contradiction. Hence Inequality (15) follows.

Finally, Inequality (16) follows from the definition of $V_{x, y}$.

4. Let $y, y^{n} \in \mathcal{C}_{x}$ with $y^{n} \rightarrow y$. By 3., the sequence $\left(\mu^{*}\left(y^{n}\right)\right)_{n}$ is bounded and hence there exists a convergent subsequence, say (by abuse of notation)

$$
\mu^{*}\left(y^{n}\right) \rightarrow \mu^{*} \quad \text { with } \mu_{j}^{*} \geq 0 \text { for all } j .
$$

In particular, we have

$$
\sum_{j} \mu_{j}^{*} h_{j}=y
$$

We have

$$
\begin{aligned}
y^{n} & =\sum_{j} \mu_{j}^{*}\left(y^{n}\right) h_{j} \\
& =\left(1-\epsilon^{n}\right) \sum_{j} \mu_{j}^{*} h_{j}+\sum_{j}\left(\mu_{j}^{*}\left(y^{n}\right)-\mu_{j}^{*}+\epsilon^{n} \mu_{j}^{*}\right) h_{j} \\
& =\left(1-\epsilon^{n}\right) \sum_{j} \mu_{j}^{*}(y) h_{j}+\sum_{j} \tilde{\mu}_{j}^{n} h_{j},
\end{aligned}
$$

where we have used (17), $\mu^{*}(y)=\operatorname{argmax}_{\mu} \ell(x, \mu), \tilde{\mu}_{j}^{n}=\mu_{j}^{*}\left(y^{n}\right)-\mu_{j}^{*}+\epsilon^{n} \mu_{j}^{*}$, and

$$
\epsilon^{n}= \begin{cases}\frac{2 \max _{j}\left|\mu_{j}^{*}\left(y^{n}\right)-\mu_{j}^{*}\right|}{\min _{j ; \mu_{j}^{*}>0} \mu_{j}^{*}}, & \text { if } \min _{j ; \mu_{j}^{*}>0} \mu_{j}^{*}>0 \\ 1 / n, & \text { otherwise. }\end{cases}
$$

In particular, we have $0 \leq \tilde{\mu}_{j}^{n} \rightarrow 0$ as $n \rightarrow \infty$. By Equation (18), 2. and the continuity of $\ell$, we have

$$
\begin{aligned}
\left.\ell\left(x, \mu^{*}\left(y^{n}\right)\right)\right) & \leq \ell\left(x,\left(1-\epsilon^{n}\right) \mu^{*}(y)+\tilde{\mu}^{n}\right) \\
& \leq \ell\left(x, \mu^{*}(y)\right)+\delta(n)
\end{aligned}
$$

with $\delta(n) \rightarrow \infty$ as $n \rightarrow \infty$. This implies (again by the continuity of $\ell$ )

$$
\ell\left(x, \mu^{*}\right) \leq \ell\left(x, \mu^{*}(y)\right)
$$

and hence $\mu^{*}=\mu^{*}(y)$ by 2 . As this holds true for all convergent subsequences of $\left(\mu^{*}\left(y^{n}\right)\right)_{n}$, this establishes the continuity of $\mu^{*}(\cdot)$.

The continuity of $\bar{L}(x, \cdot)$ follows directly from this and the continuity of $\ell$.

Remark 4.6. Assume that for $x \in A, \mathcal{C}_{x}=\mathcal{C}_{\tilde{x}}$ for all $\tilde{x}$ in some neighborhood $U$ of $x$. Then the function $\ell: U \times V_{x, y} \rightarrow \mathbb{R}_{+}$is continuous and hence we have that $\mu^{*}(y)=\mu^{*}(x, y)$ as given in Lemma 4.5 is also continuous in $x$ (as the argmin of a continuous function). 
We have moreover

Lemma 4.7. 1. Let $x \in A$. For all $B>0$, there exists a constant $C_{6}=C_{6}(x, B)>0$ such that for all $y \in \mathcal{C}_{x}$ with $|y| \leq B$ and $\theta \in \mathbb{R}^{d}$ with $\tilde{\ell}(x, y, \theta) \geq-15$

$$
\left\langle\theta, h_{j}\right\rangle \leq C_{6} \quad \text { for all } j \text { with } \beta_{j}(x)>0 \text {. }
$$

If $\log \beta_{j}(\cdot)(j=1 \ldots, k)$ is bounded, $C_{6}$ can be chosen independently of $x$.

2. Let $x \in A$ and $y \in \mathcal{C}_{x}$. If $\left(\theta_{n}\right)_{n}$ is a maximizing sequence of $\tilde{\ell}(x, y, \cdot)$ and for some $j=1, \ldots, k$,

$$
\liminf _{n \rightarrow \infty}\left\langle\theta_{n}, h_{j}\right\rangle=-\infty
$$

then

$$
\mu_{j}=0 \quad \text { for all } \mu \in V_{x, y} .
$$

Conversely, there exists a constant $\tilde{C}_{6}=\tilde{C}_{6}(B)>0$ such that if $|y| \leq B$ and $\mu_{j}>0$ for some $\mu \in V_{x, y}$, then

$$
\liminf _{n \rightarrow \infty}\left\langle\theta_{n}, h_{j}\right\rangle>-\tilde{C}_{6} .
$$

Proof. 1. Let $|y| \leq B, C_{2}$ and $\mu \in V_{x, y}$ be according to Lemma 4.4. Define the functions from $\mathbb{R}$ into itself

$$
f_{j}(z):=\mu_{j} z-\beta_{j}(x)\left(\mathrm{e}^{z}-1\right) .
$$

Note that $f_{j}(z)=0$ if $\beta_{j}(x)=0$, and $\operatorname{argmax}_{z} f_{j}(z)=\log \mu_{j} / \beta_{j}(x)$ if $\beta_{j}(x)>0$. Let

$$
\tilde{C}(x, B)=\sup _{j ; \beta_{j}(x)>0} \sup _{|\mu| \leq C_{2} B} f_{j}\left(\log \frac{\mu_{j}}{\beta_{j}(x)}\right) .
$$

If $x, y$ and $\theta$ are as in the statement, and $1 \leq j \leq k$ is such that $\beta_{j}(x)>0$ and $\left\langle\theta, h_{j}\right\rangle>0$, then

$$
\sum_{j^{\prime} \neq j} f_{j^{\prime}}\left(\left\langle\theta, h_{j^{\prime}}\right\rangle\right)=\tilde{\ell}(x, y, \theta)-f_{j}\left(\left\langle\theta, h_{j}\right\rangle\right),
$$

hence in view of the assumption,

$$
f_{j}\left(\left\langle\theta, h_{j}\right\rangle\right) \geq-1-(k-1) \tilde{C}(x, B),
$$

As $f_{j}(z) \rightarrow-\infty$ as $z \rightarrow \infty$, the assertion follows.

2. If $\liminf _{n \rightarrow \infty}\left\langle\theta_{n}, h_{j}\right\rangle=-\infty$ and $\mu \in V_{x, y}$ with $\mu_{j}>0$, then 1 . implies that $\tilde{\ell}\left(x, y, \theta_{n}\right) \rightarrow-\infty$, a contradiction.

The second assertion follows accordingly.

We now prove

\footnotetext{
${ }^{5}$ The constant -1 can be replaced by any other constant $-C(C>0)$. Note that $C_{6}$ then depends on $C$ with $C_{6}$ increasing in $C$.
} 
Lemma 4.8. 1. Let $x \in A$ and $y \in \mathcal{C}_{x}$. Then there exists a maximizing sequence $\left(\theta_{n}\right)_{n}$ of $\tilde{\ell}(x, y, \cdot)$ and constants $\tilde{s}_{j}$ (for all $j=1, \ldots, k$ for which there exists a $\mu \in V_{x, y}$ with $\left.\mu_{j}>0\right)$ such that

$$
\lim _{n \rightarrow \infty}\left\langle\theta_{n}, h_{j}\right\rangle=\tilde{s}_{j} \in \mathbb{R} .
$$

The constants $\tilde{s}_{j}$ are bounded uniformly over bounded sets of $y \in \mathcal{C}_{x}$.

In particular, there exists a maximizing sequence $\left(\theta_{n}\right)_{n}$ such that for all $j=1, \ldots, k$ with $\beta_{j}(x)>0$,

$$
\lim _{n \rightarrow \infty} \exp \left(\left\langle\theta_{n}, h_{j}\right\rangle\right)=s_{j} \in \mathbb{R} 6
$$

2. Let $x \in A$ and $y \notin \mathcal{C}_{x}$. Then $\underline{L}(x, y)=\infty$.

Proof. 1. By Lemma 4.7,

$$
-\tilde{C}_{6}=-\tilde{C}_{6}(|y|)<\left\langle\theta_{n}, h_{j}\right\rangle \leq C_{6}=C_{6}(|y|)
$$

for all $n$ and for all $j$ with $\mu_{j}>0$ for some $\mu \in V_{x, y}$. The first assertion follows by taking appropriate subsequences.

For the second assertion, we have to consider those $j$ with $\mu_{j}=0$ for all $\mu \in V_{x, y}$ although $\beta_{j}(x)>0$. If $\liminf _{n \rightarrow \infty}\left\langle\theta_{n}, h_{j}\right\rangle=-\infty$, we take further subsequences and obtain (with a slight abuse of notation)

$$
\lim _{n \rightarrow \infty} \exp \left(\left\langle\theta_{n}, h_{j}\right\rangle\right)=0 .
$$

2. Let $y \notin \mathcal{C}_{x}$ and $v$ be the projection of $y$ on $\mathcal{C}_{x}$. Hence, $0=\langle y-v, v\rangle \geq\langle y-v, \tilde{v}\rangle$ for all $\tilde{v} \in \mathcal{C}_{x}$. For $z=y-v\left(\neq 0\right.$ as $\left.y \notin \mathcal{C}_{x}\right)$, we have $\langle z, y\rangle=\langle z, z\rangle+\langle z, v\rangle>0$ and $\left\langle z, h_{j}\right\rangle \leq 0$ for all $j$ with $\beta_{j}(x)>0$. If we set $\theta_{n}=n z$, we obtain $\ell\left(x, y, \theta_{n}\right) \rightarrow \infty$.

\subsection{Equality of $\underline{L}$ and $\bar{L}$}

We can now finally establish

Theorem 4.9. For all $x \in A, y \in \mathbb{R}^{d}$,

$$
\underline{L}(x, y)=\bar{L}(x, y) .
$$

Proof. In view of Lemma 2.2, it suffices to prove that $\bar{L}(x, y \leq \underline{L}(x, y)$. We first note that we have $\underline{L}(x, y)<\infty$ if and only if $y \in \mathcal{C}_{x}$ by Lemma 4.8 2. and Lemma 4.11. As the same is true for $\bar{L}(x, y)$ by definition, we can restrict our attention to the case $y \in \mathcal{C}_{x}$.

We choose a maximizing sequence $\left(\theta_{n}\right)_{n}$ according to Lemma 4.7 and obtain

$$
\lim _{n}\left\langle\theta_{n}, y\right\rangle=\underline{L}(x, y)+\sum_{j} \beta_{j}(x)\left(s_{j}-1\right)
$$

\footnotetext{
${ }^{6}$ Note that here, we also include those $j$ with $\beta_{j}(x)>0$ and $\mu_{j}=0$ for all $\mu \in V_{x, y}$.
} 
here we set $s_{j}=0$ if $\beta_{j}(x)=0$. We now differentiate with respect to $\theta$ and obtain for all $n$

$$
\nabla_{\theta} \tilde{\ell}\left(x, y, \theta_{n}\right)=y-\sum_{j ; \beta_{j}(x)>0} \beta_{j}(x) h_{j} \exp \left(\left\langle\theta_{n}, h_{j}\right\rangle\right) ;
$$

hence (by the fact that $\left(\theta_{n}\right)_{n}$ is a maximizing sequence and the limit of $\nabla_{\theta} \tilde{\ell}\left(x, y, \theta_{n}\right)$ exists),

$$
\lim _{n} \nabla_{\theta} \tilde{\ell}\left(x, y, \theta_{n}\right)=y-\sum_{j ; \beta_{j}(x)>0} \beta_{j}(x) s_{j} h_{j}=0 .
$$

We set,

$$
\mu_{j}^{*}:=\beta_{j}(x) s_{j}
$$

in particular

$$
y=\sum_{j} \mu_{j}^{*} h_{j} \quad \text { and } \quad \mu^{*} \in V_{x, y}
$$

Therefore,

$$
\begin{aligned}
\bar{L}(x, y) & \leq \ell\left(x, \mu^{*}\right) \\
& =\sum_{j} \beta_{j}(x)-\mu_{j}^{*}+\mu_{j}^{*} \log \left(\frac{\mu_{j}^{*}}{\beta_{j}(x)}\right) \\
& =\sum_{j} \beta_{j}(x)\left(1-s_{j}\right)+\mu_{j}^{*} \log s_{j} \\
& =\underline{L}(x, y)
\end{aligned}
$$

where we have used (20) and (21) for the last identity. The assertion follows.

From now on, we shall write $L(x, y)$ for the quantity $\underline{L}(x, y)=\bar{L}(x, y)$.

We now prove the strict convexity of $L(x, \cdot)$.

Corollary 4.10. For all $x \in A, L(x, \cdot): \mathcal{C}_{x} \rightarrow \mathbb{R}_{+}$is strictly convex.

Proof. For strict convexity, we exclude the case that $\beta_{j}(x)=0$ for all $j$ (as then $L(x, y)=$ $\infty$ for all $y \neq 0$ and the assertion is trivial).

Convexity was proven in Lemma 4.1. Assume now that for $y, \tilde{y} \in \mathcal{C}_{x}$ and $\lambda \in(0,1)$,

$$
L(x, \lambda y+(1-\lambda) \tilde{y})=\lambda L(x, y)+(1-\lambda) L(x, \tilde{y})
$$

In other words,

$$
\begin{aligned}
\sup _{\theta} & \left\{\langle\theta, \lambda y+(1-\lambda) \tilde{y}\rangle-\sum_{j} \beta_{j}(x)\left(\mathrm{e}^{\left\langle\theta, h_{j}\right\rangle}-1\right)\right\} \\
& =\sup _{\theta}\left\{\lambda\left[\langle\theta, y\rangle-\sum_{j} \beta_{j}(x)\left(\mathrm{e}^{\left\langle\theta, h_{j}\right\rangle}-1\right)\right]+(1-\lambda)\left[\langle\theta, \tilde{y}\rangle-\sum_{j} \beta_{j}(x)\left(\mathrm{e}^{\left\langle\theta, h_{j}\right\rangle}-1\right)\right]\right\} \\
& =\lambda \sup _{\theta}\left\{\langle\theta, y\rangle-\sum_{j} \beta_{j}(x)\left(\mathrm{e}^{\left\langle\theta, h_{j}\right\rangle}-1\right)\right\}+(1-\lambda) \sup _{\theta}\left\{\langle\theta, \tilde{y}\rangle-\sum_{j} \beta_{j}(x)\left(\mathrm{e}^{\left\langle\theta, h_{j}\right\rangle}-1\right)\right\} .
\end{aligned}
$$


Hence, if $\left(\theta_{n}\right)_{n}$ is a maximizing sequence for $\tilde{\ell}(x, \lambda y+(1-\lambda) \tilde{y}, \cdot)$, it is also a maximizing sequence for $\tilde{\ell}(x, y, \cdot)$ and $\tilde{\ell}(x, \tilde{y}, \cdot)$. As in the proof of Theorem 4.9, this implies

$$
\begin{aligned}
& \lim _{n \rightarrow \infty} \nabla_{\theta} \tilde{\ell}\left(x, y, \theta_{n}\right)=y-\lim _{n \rightarrow \infty} \sum_{j} \beta_{j}(x)\left(\mathrm{e}^{\left\langle\theta_{n}, h_{j}\right\rangle}-1\right)=0, \\
& \lim _{n \rightarrow \infty} \nabla_{\theta} \tilde{\ell}\left(x, \tilde{y}, \theta_{n}\right)=\tilde{y}-\lim _{n \rightarrow \infty} \sum_{j} \beta_{j}(x)\left(\mathrm{e}^{\left\langle\theta_{n}, h_{j}\right\rangle}-1\right)=0 .
\end{aligned}
$$

Hence $y=\tilde{y}$ as required.

\subsection{Further properties of the Legendre Fenchel transform}

In this subjection, we assume that the $\log \beta_{j}$ 's are bounded. In this case $\mathcal{C}_{x}=\mathcal{C}=\mathbb{R}^{d}$ for all $x$.

We have

Lemma 4.11. Assume that $\log \beta_{j}(j=1, \ldots, k)$ is bounded.

1. For all $B>0$ exists a constant $C_{7}=C_{7}(B)>0$ such that for all $x \in A, y \in \mathcal{C}$ with $|y| \leq B$

$$
L(x, y) \leq C_{7}
$$

2. For all $x \in A, L(x, \cdot): \mathcal{C} \rightarrow \mathbb{R}_{+}$is continuous.

Proof. 1. Let $x \in A, y \in \mathcal{C}$. By Lemma 4.4 and Theorem 4.9 below7, we obtain

$$
\begin{aligned}
L(x, y) & \leq \sum_{j, \beta_{j}(x)>0} \beta_{j}(x)-\mu_{j}+\mu_{j} \log \mu_{j}-\mu_{j} \log \beta_{j}(x) \\
& \leq k(\bar{\beta}+C|y| \log C+C|y| \log |y|+C|y||\log \underline{\beta}|) .
\end{aligned}
$$

The assertion follows.

2. The assertion follows directly from 1., Lemma 4.11 .

We have moreover

Lemma 4.12. Assume that $\log \beta_{j}(j=1, \ldots, k)$ is bounded. For all $\rho>0, \epsilon>0, C_{8}>0$, there exists a constant $B_{3}=B\left(C_{8}, \epsilon\right)$ such that for all $x \in A, y \in \mathcal{C}$ with $|y| \leq C_{8}$,

$$
\sup _{|\theta| \leq B} \tilde{\ell}(x, y, \theta) \geq \sup _{\theta \in \mathbb{R}^{d}} \tilde{\ell}(x, y, \theta)-\epsilon=L(x, y)-\epsilon .
$$

Proof. We first fix $x \in A$ and define the compact set

$$
\tilde{\mathcal{C}}:=\left\{y \in \mathcal{C}|| y \mid \leq C_{8}\right\}
$$

We fix $\delta>0$ and define for $y \in \tilde{\mathcal{C}}$,

$$
z(y, \delta):=y+\sum_{j} \delta h_{j}
$$

\footnotetext{
${ }^{7}$ note that this result is not used for the proof of Theorem 4.9.
} 


$$
N^{y, \delta}:=\left\{y+\sum_{j} \alpha_{j} h_{j} \mid \alpha_{j} \in(-\delta, \delta)\right\} .
$$

For all $y \in \tilde{\mathcal{C}}, N^{y, \delta}$ is relatively open (with respect to $\mathcal{C}$ ) and $y \in N^{y, \delta}$. Hence there exists a finite cover $N_{1}, \ldots, N_{n}$ of $\tilde{\mathcal{C}}$, where $N_{i}:=N^{y_{i}, \delta}$ for appropriate $y_{i} \in \tilde{\mathcal{C}}$; we define $z_{i}:=z\left(y_{i}, \delta\right)$.

We use the continuity of $L(x, \cdot): \mathcal{C} \rightarrow \mathbb{R}_{+}(\operatorname{cf}$. Lemma 4.11 2.) and the fact that $\tilde{\mathcal{C}}$ is compact, we obtain for $\delta$ small enough that for all $y \in \tilde{\mathcal{C}}, v \in N^{y, \delta}$,

$$
|L(x, v)-L(x, z(y, \delta))|<\frac{\epsilon}{4} .
$$

We let $\theta_{i}$ be almost optimal for $z_{i}$ in the sense that

$$
\tilde{\ell}\left(x, z_{i}, \theta_{i}\right) \geq L\left(x, z_{i}\right)-\frac{\epsilon}{4} .
$$

We now set $B^{x}:=\max _{i}\left|\theta_{i}\right|$ and let $y \in \tilde{\mathcal{C}}$, say $y \in N_{i}$. Then, making use successively of (22) and (23), we obtain

$$
\begin{aligned}
L(x, y) & \leq L\left(x, z_{i}\right)+\frac{\epsilon}{4} \\
& \leq \tilde{\ell}\left(x, z_{i}, \theta_{i}\right)+\frac{\epsilon}{2} \\
& =\tilde{\ell}\left(x, y, \theta_{i}\right)+\frac{\epsilon}{2}+\left\langle\theta_{i}, z_{i}-y\right\rangle .
\end{aligned}
$$

We have $z_{i}-y=\sum_{j} \mu_{j} h_{j}$ for appropriate $\mu_{j}=\alpha_{j}+\delta \in(0,2 \delta)$ and by Lemma 4.7 (cf. also Inequality (22) $),\left\langle\theta_{i}, h_{j}\right\rangle \leq C_{6}$. Hence

$$
\left\langle z_{i}-y, \theta_{i}\right\rangle=\sum_{j} \mu_{j}\left\langle h_{j}, \theta_{i}\right\rangle \leq 2 k C_{6} \delta \leq \frac{\epsilon}{4},
$$

provided we choose $\delta$ such that $8 k C_{6} \delta \leq \epsilon$. Therefore by Inequalities (24) and (25) for all $y \in \tilde{\mathcal{C}}$

$$
\left.L(x, y) \leq \tilde{\ell}\left(x, y, \theta_{i}\right)+\epsilon \quad \text { (recall that }\left|\theta_{i}\right| \leq B^{x}\right) .
$$

Let now for all $x \in A, B^{x}$ be the bound obtained above belonging to $\frac{\epsilon}{4} 8$ Let furthermore $x, \tilde{x} \in A$ with $|\beta(x)-\beta(\tilde{x})|<\delta$ for some $\delta>0, y \in \tilde{\mathcal{C}},|y| \leq C_{8}$ and $\theta \in \mathbb{R}^{d}$ such that $\tilde{\ell}(x, y, \theta) \geq-1$ (which implies $\left\langle\theta, h_{j}\right\rangle \leq C_{6}$ by Lemma 4.7). This implies

$$
|\tilde{\ell}(x, y, \theta)-\tilde{\ell}(\tilde{x}, y, \theta)| \leq \sum_{j}\left|\beta_{j}(x)-\beta_{j}(\tilde{x})\right| \mathrm{e}^{C_{6}}<\frac{\epsilon}{4}
$$

for $\delta$ small enough (and independent of $x, \tilde{x}, y, \theta)$. Let now be $\tilde{\theta}$ be almost optimal for $\tilde{x}, y$. Using twice (27) and once (26), we obtain

$$
\begin{aligned}
L(\tilde{x}, y) & \leq \tilde{\ell}(\tilde{x}, y, \tilde{\theta})+\frac{\epsilon}{4} \\
& \leq \tilde{\ell}(x, y, \tilde{\theta})+\frac{\epsilon}{2}
\end{aligned}
$$

\footnotetext{
${ }^{8}$ Note that $B^{x}$ depends on $x$ only through $\beta(x)$.
} 


$$
\begin{aligned}
& \leq \sup _{|\theta| \leq B^{x}} \tilde{\ell}(x, y, \theta)+\frac{3 \epsilon}{4} \\
& \leq \sup _{|\theta| \leq B^{x}} \tilde{\ell}(\tilde{x}, y, \theta)+\epsilon
\end{aligned}
$$

We can cover the compact interval $[\beta, \bar{\beta}]$ by finitely many $\tilde{\delta}$-neighborhoods of $\beta^{i}$. The assertion follows by taking the maximum of the corresponding $B^{i}$ (cf. Footnote 8 ).

Lemma 4.13. Assume that $\log \beta_{j}(j=1, \ldots, k)$ is bounded. There exist constants $B_{4}$ and $C_{9}$ such that for all, $x \in A$ and $y \in \mathcal{C}$,

$$
L(x, y) \leq \begin{cases}C_{9} & \text { if }|y| \leq B_{4} \\ C_{9}|y| \log |y| & \text { if }|y|>B_{4}\end{cases}
$$

Proof. From the formula for $\bar{L}(x, y)$ and Lemma 4.4, we have

$$
\begin{aligned}
L(x, y) & \leq \sum_{j} \bar{\beta}+C|y| \log |y|+C|y||\log \underline{\beta}| \\
& \leq k \cdot(\bar{\beta}+C|y| \log |y|+C|y||\log \underline{\beta}|) .
\end{aligned}
$$

We also obtain the continuity of $L$ in $x$

Lemma 4.14. Assume that $\log \beta_{j}(j=1, \ldots, k)$ is bounded and continuous. For all $y \in \mathcal{C}$,

$$
L(\cdot, y): A \rightarrow \mathbb{R}_{+}
$$

is continuous. The continuity is uniform over bounded $y$.

Proof. We let $y \in \mathcal{C}$ with $|y| \leq B, 0<\epsilon<1$. and $x, \tilde{x} \in A$. Let $\theta$ such that

$$
L(x, y) \leq \tilde{\ell}(x, y, \theta)+\frac{\epsilon}{2}
$$

We have by the continuity of the $\beta_{j}$ and Lemma 4.7,

$$
|\tilde{\ell}(x, y, \theta)-\tilde{\ell}(\tilde{x}, y, \theta)| \leq \sum_{j}\left|\beta_{j}(x)-\beta_{j}(\tilde{x})\right| e^{C_{6}}<\frac{\epsilon}{2}
$$

if $|x-\tilde{x}|<\delta$ for appropriate $\delta>0$ (independent of $x, \tilde{x} \in A$ and $y$ with $|y| \leq B$ ). Thus,

$$
L(x, y) \leq \tilde{\ell}(\tilde{x}, y, \theta)+\epsilon \leq L(\tilde{x}, y)+\epsilon
$$

Reversing the roles of $x$ and $\tilde{x}$ proves the assertion.

Combinig Lemma 4.14 and Lemma 4.11, we deduce the

Corollary 4.15. Assume that $\log \beta_{j}(j=1, \ldots, k)$ is bounded and continuous. Then $L: A \times \mathbb{R}^{d} \rightarrow \mathbb{R}_{+}$is continuous. 


\subsection{The rate function}

Recall that for $\phi:[0, T] \rightarrow A$, we let

$$
I_{T}(\phi):= \begin{cases}\int_{0}^{T} L\left(\phi(t), \phi^{\prime}(t)\right) d t & \text { if } \phi \text { is absolutely continuous } \\ \infty & \text { otherwise. }\end{cases}
$$

For $x \in A$ and $\phi:[0, T] \rightarrow A$, let

$$
I_{T, x}(\phi):= \begin{cases}I_{T}(\phi) & \text { if } \phi(0)=x \\ \infty & \text { otherwise }\end{cases}
$$

We first have the following statement, which follows readily from point 2 in Lemma 4.1 .

Lemma 4.16. Assume that $x \in A$. If $\phi$ solves the $O D E(2)$, then $I_{T, x}(\phi)=0$. Conversely, if the ODE (2) admits a unique solution $Y^{x}$ and $I_{T, x}(\phi)=0$, then $\phi(t)=Y^{x}(t)$ for all $t \in[0, T]$.

In the next statement, $B_{1}$ refers to the constant appearing in Lemma 4.3.

Lemma 4.17. Assume that $\beta_{j}(j=1, \ldots, k)$ is bounded.

1. Let $K, \epsilon>0$. There exits $\delta>0$ such that for all $\phi$ with $I_{T, x}(\phi) \leq K$ and for all finite collections of non-overlapping subintervals of $[0, T],\left[s_{1}, t_{1}\right], \ldots,\left[s_{J}, t_{J}\right]$, with $\sum_{i}\left(t_{i}-s_{i}\right)=\delta$

$$
\sum_{i}\left|\phi\left(t_{i}\right)-\phi\left(s_{i}\right)\right|<\epsilon
$$

2. Let $K>0$. Then, for all constants $B \geq B_{1}$ and for all $\phi$ with $I_{T, x}(\phi) \leq K$,

$$
\int_{0}^{T} \mathbb{1}_{\left\{\left|\phi^{\prime}(t)\right| \geq B\right\}} d t<\frac{K}{C_{1} B \log B} .
$$

Proof. 1. Note first that

$$
f(\alpha):=\inf _{x, y}\left\{\frac{L(x, y)}{|y|}|| y \mid \geq \alpha\right\} \rightarrow \infty
$$

as $\alpha \rightarrow \infty$ by Lemma 4.3. For $g(t):=\mathbb{1}_{\cup_{j}\left[s_{j}, t_{j}\right]}$ and $\alpha=1 / \sqrt{\delta}$, we obtain by the fact that $\phi$ is absolutely continuous

$$
\begin{aligned}
\sum_{j}\left|\phi\left(t_{j}\right)-\phi\left(s_{j}\right)\right| & \leq \int_{0}^{T}\left|\phi^{\prime}(t)\right| g(t) d t \\
& \leq \int_{0}^{T} \alpha \mathbb{1}_{\left\{\left|\phi^{\prime}(t)\right| \leq \alpha\right\}} g(t) d t+\int_{0}^{T} \mathbb{1}_{\left\{\left|\phi^{\prime}(t)\right|>\alpha\right\}} \frac{L\left(\phi(t), \phi^{\prime}(t)\right)}{L\left(\phi(t), \phi^{\prime}(t)\right) /\left|\phi^{\prime}(t)\right|} g(t) d t \\
& \leq \alpha \delta+\frac{I_{T, x}(\phi)}{f(\alpha)} \\
& \leq \sqrt{\delta}+\frac{K}{f(1 / \sqrt{\delta})} \rightarrow 0,
\end{aligned}
$$

as $\delta \rightarrow 0$. 
2. For $B>0$, we define the function

$$
f(B):=\inf _{x, y}\left\{\frac{L(x, y)}{|y|}|| y \mid \geq B\right\} .
$$

By Lemma 4.3, $f(B) \geq C_{1} \log B$ for $B \geq B_{1}$.

$$
\begin{aligned}
\int_{0}^{T} \mathbb{1}_{\left\{\left|\phi^{\prime}(t)\right| \geq B\right\}} d t & \leq \frac{1}{B} \int_{0}^{T}\left|\phi^{\prime}(t)\right| \mathbb{1}_{\left\{\left|\phi^{\prime}(t)\right| \geq B\right\}} d t \\
& =\frac{1}{B} \int_{0}^{T} \frac{L\left(\phi(t), \phi^{\prime}(t)\right)\left|\phi^{\prime}(t)\right|}{L\left(\phi(t), \phi^{\prime}(t)\right)} \mathbb{1}_{\left\{\left|\phi^{\prime}(t)\right| \geq B\right\}} d t \\
& \leq \frac{K}{B f(B)} \leq \frac{K}{C_{1} B \log B} .
\end{aligned}
$$

Theorem 4.18. Assume that $\log \beta_{j}(j=1, \ldots, k)$ is bounded and continuous. Let $\phi \in$ $D([0, T] ; A)$ with $I_{T, x}(\phi)<\infty$. For all $\epsilon>0$, there exists a $\delta>0$ such that for

$$
\begin{gathered}
\tilde{\phi}:[0, T] \rightarrow A \text { with } \sup _{0 \leq t \leq T}|\tilde{\phi}(t)-\phi(t)|<\delta, \\
\left|\int_{0}^{T}\left(L\left(\tilde{\phi}(t), \phi^{\prime}(t)\right)-L\left(\phi(t), \phi^{\prime}(t)\right)\right) d t\right|<\epsilon .
\end{gathered}
$$

Proof. We choose $B \geq B_{1} \vee B_{4}$ large enough such that for $x \in A, y \in \mathcal{C}_{x}=\mathcal{C}$ (independent of $x$ ) with $|y| \geq B$ (cf. Lemmas 4.3 and 4.13),

$$
C_{1}|y| \log |y| \leq L(x, y) \leq C_{9}|y| \log |y| \text {. }
$$

As $I_{T, x}(\phi)<\infty$, the set $\left\{t \mid \phi^{\prime}(t) \notin \mathcal{C}\right\}$ is a Lebesgue null-set and we assume w.l.o.g. that for all $t, \phi^{\prime}(t) \in \mathcal{C}$. We hence obtain that

$$
\begin{aligned}
\int_{0}^{T} L\left(\tilde{\phi}(t), \phi^{\prime}(t)\right) \mathbb{1}_{\left\{\left|\phi^{\prime}(t)\right| \geq B\right\}} d t & \leq \int_{0}^{T} C_{9}\left|\phi^{\prime}(t)\right| \log \left|\phi^{\prime}(t)\right| \mathbb{1}_{\left\{\left|\phi^{\prime}(t)\right| \geq B\right\}} d t \\
& \leq \frac{C_{9}}{C_{1}} \int_{0}^{T} L\left(\phi(t), \phi^{\prime}(t)\right) \mathbb{1}_{\left\{\left|\phi^{\prime}(t)\right| \geq B\right\}} d t .
\end{aligned}
$$

From this and Lemma 4.17, we can choose $B$ large enough such that

$$
\sup \left(\int_{0}^{T} L\left(\tilde{\phi}(t), \phi^{\prime}(t)\right) \mathbb{1}_{\left\{\left|\phi^{\prime}(t)\right| \geq B\right\}} d t, \int_{0}^{T} L\left(\phi(t), \phi^{\prime}(t)\right) \mathbb{1}_{\left\{\left|\phi^{\prime}(t)\right| \geq B\right\}} d t\right)<\frac{\epsilon}{4} .
$$

By Lemma 4.14, there exists an $\delta>0$ such that for all $x, \tilde{x} \in A$ with $|x-\tilde{x}|<\delta$ and $y \in \mathcal{C}$ with $|y| \leq B$

$$
|L(x, y)-L(\tilde{x}, y)|<\frac{\epsilon}{2 T}
$$


We obtain for $\sup _{0 \leq t \leq T}|\tilde{\phi}(t)-\phi(t)|<\delta$,

$$
\begin{aligned}
\mid \int_{0}^{T} & \left(L\left(\tilde{\phi}(t), \phi^{\prime}(t)\right)-L\left(\phi(t), \phi^{\prime}(t)\right)\right) d t \mid \\
\leq & \left|\int_{0}^{T} L\left(\tilde{\phi}(t), \phi^{\prime}(t)\right) \mathbb{1}_{\left\{\left|\phi^{\prime}(t)\right| \geq B\right\}} d t\right|+\left|\int_{0}^{T} L\left(\phi(t), \phi^{\prime}(t)\right) \mathbb{1}_{\left\{\left|\phi^{\prime}(t)\right| \geq B\right\}} d t\right| \\
& \quad+\int_{0}^{T}\left|L\left(\tilde{\phi}(t), \phi^{\prime}(t)\right)-L\left(\phi(t), \phi^{\prime}(t)\right)\right| \mathbb{1}_{\left\{\left|\phi^{\prime}(t)\right|<B\right\}} d t \\
& <\epsilon .
\end{aligned}
$$

\section{5 $I$ is a good rate function}

We first have

Lemma 4.19. For $\delta>0, x \in A$ and $y \in \mathbb{R}^{d}$, we define

$$
L_{\delta}(x, y):=\sup _{\theta \in \mathbb{R}^{d}} \tilde{\ell}_{\delta}(x, y, \theta),
$$

where

$$
\tilde{\ell}_{\delta}(x, y, \theta):=\langle\theta, y\rangle-\sup _{z \in A ;|z-x| \leq \delta} \sum_{j} \beta_{j}(z)\left(\mathrm{e}^{\left\langle\theta, h_{j}\right\rangle}-1\right) .
$$

Since the $\beta_{j}$ are bounded and continuous, then

$$
L_{\delta}(x, y) \uparrow L_{0}(x, y)=L(x, y)
$$

and $L_{\delta}(x, y)$ is lower semicontinuous in $(\delta, x, y)$.

Proof. It is easy to see that $\tilde{\ell}_{\delta}(x, y, \theta)$ is continuous is $(x, y, \delta)$, hence the first assertion follows. The second assertion follows from the fact that the supremum of a family of lower semicontinuous functions is lower semicontinuous.

We next establish (recall the metric $d_{D}$ introduced in subsection 2.1)

Lemma 4.20. Let the $\beta_{j}$ be bounded and continuous. Then, $I_{T}$ is lower semicontinuous with respect to the metric $d_{D}$ on $D([0, T] ; A)$.

Proof. As $I_{T}(\phi)=\infty$ if $\phi$ is not absolutely continuous, we can restrict our attention to sequences of absolutely continuous functions. As the Skorohod topology relativized to $C([0, T] ; A)$ coincides with the uniform topology (see, e.g., Billingsley [1999], Section 12 , p.124), we can consider a sequence of functions $\phi_{n} \in C([0, T] ; A)$ converging to a function $\phi$ under the uniform topology. We can furthermore assume that $I_{T}\left(\phi_{n}\right) \leq K$ for some $K$ and all $n \geq 1$. By Lemma 4.17, the functions $\phi_{n}$ are hence uniformly absolutely continuous and therefore the limit $\phi$ is absolutely continuous.

Therefore, for any given $\delta>0$, there exists a $\Delta>0$ such that

$$
|s-t| \leq \Delta \Rightarrow\left|\phi_{n}(s)-\phi_{n}(t)\right| \leq \delta \text { for all } n .
$$


We choose $\Delta$ smaller if necessary such that $T / \Delta=: J \in \mathbb{N}$ and divide $[0, T]$ into subintervals $\left[t_{j}, t_{j+1}\right], j=1, \ldots, J$ of length $\leq \Delta$. We note that for $\left|x^{\prime}-x\right| \leq \delta$, we have $L_{\delta}\left(x^{\prime}, y\right) \leq L(x, y)$. Furthermore, we observe that $L_{\delta}(x, \cdot)$ is convex as a supremum of linear functions and hence by Jensen's Inequality

$$
\begin{aligned}
\int_{0}^{T} L\left(\phi_{n}(t), \phi_{n}^{\prime}(t)\right) d t & \geq \sum_{j=1}^{J} \int_{t_{j}}^{t_{j+1}} L_{\delta}\left(\phi_{n}\left(t_{j}\right), \phi_{n}^{\prime}(t)\right) d t \\
& \geq \sum_{j=1}^{J} \Delta L_{\delta}\left(\phi_{n}\left(t_{j}\right), \frac{\phi_{n}\left(t_{j+1}\right)-\phi_{n}\left(t_{j}\right)}{\Delta}\right) .
\end{aligned}
$$

We now further divide the interval $[0, T]$ into subintervals of length $\Delta_{k}:=\Delta 2^{-k}, k \in \mathbb{N}$, $\left[t_{j}^{k}, t_{j+1}^{k}\right], j=1, \ldots, J_{k}:=2^{k} J$ and define the functions

$$
\underline{\phi}^{k}(t):=\phi\left(t_{j}^{k}\right) \quad \text { if } t \in\left[t_{j}^{k}, t_{j+1}^{k}\right], \quad \bar{\phi}^{k}(t):=\underline{\phi}^{k}\left(t+\Delta_{k}\right) .
$$

Note that there exits a sequence $\delta_{k} \downarrow 0$ such that

$$
|s-t|<\Delta_{k} \Rightarrow\left|\phi_{n}(s)-\phi_{n}(t)\right|<\delta_{k} \text { for all } n .
$$

Hence by Inequality (28) and Lemma 4.19 for all $k \in \mathbb{N}$,

$$
\begin{aligned}
\liminf _{n \rightarrow \infty} \int_{0}^{T} L\left(\phi_{n}(t), \phi_{n}^{\prime}(t)\right) d t & \geq \sum_{j=1}^{J_{k}} \Delta_{k} \liminf _{n \rightarrow \infty} L_{\delta_{k}}\left(\phi_{n}\left(t_{j}^{k}\right), \frac{\phi_{n}\left(t_{j+1}^{k}\right)-\phi_{n}\left(t_{j}^{k}\right)}{\Delta_{k}}\right) \\
& \geq \int_{0}^{T-\Delta_{k}} L_{\delta_{k}}\left(\underline{\phi}^{k}(t), \frac{\bar{\phi}^{k}(t)-\underline{\phi}^{k}(t)}{\Delta_{k}}\right) d t .
\end{aligned}
$$

As $\phi$ is absolutely continuous, we have that for almost all $t \in[0, T]$,

$$
\frac{\bar{\phi}^{k}(t)-\underline{\phi}^{k}(t)}{\Delta_{k}} \rightarrow \phi^{\prime}(t) \quad \text { as } k \rightarrow \infty .
$$

We conclude by using Inequality (29), Fatou's Lemma and Lemma 4.19 again:

$$
\begin{aligned}
\liminf _{n \rightarrow \infty} \int_{0}^{T} L\left(\phi_{n}(t), \phi_{n}^{\prime}(t)\right) d t & \geq \liminf _{k \rightarrow \infty} \int_{0}^{T-\Delta_{k}} L_{\delta_{k}}\left(\underline{\phi}^{k}(t), \frac{\bar{\phi}^{k}(t)-\underline{\phi}^{k}(t)}{\Delta_{k}}\right) d t \\
& \geq \int_{0}^{T} \liminf _{k \rightarrow \infty}\left(\mathbb{1}_{\left[0, T-\Delta_{k}\right]}(t) L_{\delta_{k}}\left(\underline{\phi}^{k}(t), \frac{\bar{\phi}^{k}(t)-\underline{\phi}^{k}(t)}{\Delta_{k}}\right)\right) d t \\
& \geq \int_{0}^{T} L\left(\phi(t), \phi^{\prime}(t)\right) d t
\end{aligned}
$$

as required.

We define for $K>0, x \in A$,

$$
\begin{aligned}
\Phi(K) & =\left\{\phi \in D([0, T] ; A) \mid I_{T}(\phi) \leq K\right\}, \\
\Phi_{x}(K) & =\left\{\phi \in D([0, T] ; A) \mid I_{T, x}(\phi) \leq K\right\} .
\end{aligned}
$$

We have moreover 
Proposition 4.21. Assume that $\beta_{j}(j=1, \ldots, k)$ are bounded and continuous. Let furthermore $K>0$ and $\tilde{A} \subset A$ be compact. Then, the sets

$$
\bigcup_{x \in \tilde{A}} \Phi_{x}(K)
$$

are compact in $C([0, T] ; A)$.

Proof. By Lemma 4.17, the functions in $\bigcup_{x \in \tilde{A}} \Phi_{x}(K)$ are equicontinuous. As $\tilde{A}$ is compact, the Theorem of Arzelà-Ascoli hence implies that $\bigcup_{x \in \tilde{A}} \Phi_{x}(K)$ has compact closure. Now, the semicontinuity of $I$ (cf. Lemma 4.20) implies that $\bigcup_{x \in \tilde{A}} \Phi_{x}(K)$ is closed which finishes the proof.

We define for $S \subset D([0, T] ; A)$,

$$
I_{x}(S):=\inf _{\phi \in S} I_{T, x}(\phi) .
$$

Lemma 4.22. Assume that $\beta_{j}(j=1, \ldots, k)$ are bounded and continuous. Let $F \subset$ $C([0, T] ; A)$ be closed. Then $I_{x}(F)$ is lower semicontinuous in $x$.

Proof. We let $x_{n} \rightarrow x$ with $\liminf _{n \rightarrow \infty} I_{x_{n}}(F)=: K<\infty$. For simplicity, we assume that $I_{x_{n}}(F) \leq K+\epsilon$ for some fixed $\epsilon>0$ and for all $n$. By Proposition 4.21, we have that for all $\epsilon, \delta>0$,

$$
F \cap \Phi_{x_{n}}(K+\epsilon) \text { and } F \cap \bigcup_{|x-y| \leq \delta} \Phi_{y}(K+\epsilon)
$$

are compact. By the semicontinuity of $I_{T}(\cdot)$ (cf. Lemma 4.20) and the fact that a l.s.c. function attains its minimum on a compact set, there exist $\phi_{n} \in F$ such that $I_{x_{n}}(F)=$ $I_{T, x_{n}}\left(\phi_{n}\right)$ (for $n$ large enough). As the $\phi_{n}$ are in a compact set, there exists a convergent subsequence with limit $\phi$, in particular $\phi(0)=x$. As $F$ is closed, we have $\phi \in F$. We use Lemma 4.20 again and obtain

$$
I_{x}(F) \leq I_{T}(\phi) \leq \liminf _{n \rightarrow \infty} I_{T}\left(\phi_{n}\right)=\liminf _{n \rightarrow \infty} I_{x_{n}}(F)=K
$$

as required.

The following result is a direct consequence of Lemma 4.22 .

Lemma 4.23. Assume that $\beta_{j}(j=1, \ldots, k)$ is bounded and continuous. For $F \subset$ $D([0, T] ; A)$ closed and $x \in A$, we have

$$
\lim _{\epsilon \downarrow 0} \inf _{y \in A,|x-y|<\epsilon} I_{y}(F)=I_{x}(F)
$$

We can now establish the main result of this subsection.

Proposition 4.24. Let the $\beta_{j}$ be bounded and continuous. For all $x, I_{x}$ is a good rate function on $C([0, T] ; A) \cap\{\phi \mid \phi(0)=x\}$.

Proof. It is clear that $I_{T}$ is non-negative as $L$ is non-negative. Furthermore, it is lower semicontinuous by Lemma 4.20, By Proposition 4.21 its level sets are compact. 
We have moreover

Corollary 4.25. Let the $\beta_{j}$ be bounded and continuous. For all $x \in A, I_{T, x}$ is a good rate function on $D([0, T] ; A) \cap\{\phi \mid \phi(0)=x\}$ under both metrics $d_{C}$ and $d_{D}$.

Proof. Since $I_{t, x}$ is finite only for absolutely continuous functions, it suffices to consider sequences in $C([0, T] ; A) \cap\{\phi \mid \phi(0)=x\}$. Limits of such sequences (under either metric) are continuous and convergence is equivalent for both metrics (see, e.g., Billingsley [1999]). Lower semicontinuity follows. Compactness of the level sets follows by Proposition 4.24 and the fact that the identity maps from $\left(C([0, T] ; A), d_{C}\right)$ into $\left(D([0, T] ; A), d_{C}\right)$ and $\left(D([0, T] ; A), d_{D}\right)$ are continuous .

\subsection{A property of non-exponential equivalence}

It is worth wondering whether or not $\left\{Z_{t}^{N, x_{N}}, 0 \leq t \leq T\right\}$ and $\left\{Z_{t}^{N, x}, 0 \leq t \leq T\right\}$ are exponentially equivalent, whenever $x_{N} \rightarrow x$ as $N \rightarrow \infty$. Indeed, Dembo and Zeitouni [2009] prove that property for diffusions with small noise and Lipschitz coefficients, and use it to establish certain results, of which we shall prove analogs below, but without that exponential equivalence, which fails to hold in our Poissonian case.

Let $x, y \in A$, and consider the processes

$$
\begin{aligned}
& Z_{t}^{N, x}=x+\sum_{j=1}^{k} \frac{h_{j}}{N} P_{j}\left(N \int_{0}^{t} \beta_{j}\left(Z_{s}^{N, x}\right) d s\right), \\
& Z_{t}^{N, y}=y+\sum_{j=1}^{k} \frac{h_{j}}{N} P_{j}\left(N \int_{0}^{t} \beta_{j}\left(Z_{s}^{N, y}\right) d s\right) .
\end{aligned}
$$

For any $\delta>0$, as $|x-y| \rightarrow 0$, we ask what is the limit, as $|x-y| \rightarrow 0$, of

$$
\limsup _{N \rightarrow \infty} \frac{1}{N} \log \mathbb{P}\left(\sup _{0 \leq t \leq T}\left|Z_{t}^{N, x}-Z_{t}^{N, y}\right|>\delta\right) ?
$$

If that limit is $-\infty$, then we would have the above exponential equivalence. We now show on a particularly simple example that this is not the case. It is easy to infer that it in fact fails in the above generality, assuming that the $\beta_{j}$ 's are Lipschitz continuous and bounded. We consider the case $d=1, A=\mathbb{R}_{+}, k=1, \beta(x)=x, h=1$. We could truncate $\beta(x)$ to make it bounded, in order to comply with our standing assumptions. The modifications below would be minor, but we prefer to keep the simplest possible notations. Assume $0<x<y$ and consider the two processes

$$
\begin{aligned}
& Z_{t}^{N, x}=x+\frac{1}{N} P\left(N \int_{0}^{t} Z_{s}^{N, x} d s\right), \\
& Z_{t}^{N, y}=y+\frac{1}{N} P\left(N \int_{0}^{t} Z_{s}^{N, y} d s\right) .
\end{aligned}
$$

It is plain that $0<Z_{t}^{N, x}<Z_{t}^{N, y}$ for all $N \geq 1$ and $t>0$. Let $\Delta_{t}^{N, x, y}=Z_{t}^{N, y}-Z_{t}^{N, x}$. The law of $\left\{\Delta_{t}^{N, x, y}, 0 \leq t \leq T\right\}$ is the same as that of the solution of

$$
\Delta_{t}^{N, x, y}=y-x+\frac{1}{N} P\left(N \int_{0}^{t} \Delta_{s}^{N, x, y} d s\right) .
$$


We deduce from Theorem 5.10 below (which is established in case of a bounded coefficient $\beta(x)$, but it makes no difference here) that

$$
\begin{aligned}
\liminf _{N \rightarrow \infty} \frac{1}{N} \log \mathbb{P}\left[\Delta_{1}^{N, x, y}>1\right] & \geq-\inf _{\phi(0)=y-x, \phi(1)>1} I_{1, y-x}(\phi) \\
& \geq-I_{1, y-x}(\psi),
\end{aligned}
$$

with $\psi(t)=y-x+t$, hence

$$
\begin{aligned}
I_{1, y-x}(\psi) & =\int_{0}^{1} L(y-x+t, 1) d t \\
& =\int_{0}^{1}[y-x+t-1-\log (y-x+t)] d t \\
& =y-x+1 / 2-(y-x+1) \log (y-x+1)+(y-x) \log (y-x) \\
& \rightarrow 1 / 2,
\end{aligned}
$$

as $y-x \rightarrow 0$. This clearly contradicts the exponential equivalence.

We note that the above process $Z_{t}^{N, x}$ can be shown to be "close" (in a sense which is made very precise in Kurtz [1978]) to its diffusion approximation

$$
X_{t}^{N, x}=x+\int_{0}^{t} X_{s}^{N, x} d s+\frac{1}{\sqrt{N}} \int_{0}^{t} \sqrt{X_{s}^{N, x}} d B_{s},
$$

where $\left\{B_{t}, t \geq 0\right\}$ is standard Brownian motion. One can study large deviations of this diffusion process from its Law of Large Numbers limit (which is the same as that of $Z_{t}^{N, x}$ ). The rate function on the time interval $[0,1]$ is now

$$
I(\phi)=\int_{0}^{1} \frac{\left(\phi^{\prime}(t)-\phi(t)\right)^{2}}{\phi(t)} d t
$$

Let again $\psi(t)=y-x+t$, now with $0=x<y . I(\psi)=\log (1+y)-\log (y)-3 / 2+y \rightarrow$ $+\infty$, as $y \rightarrow 0$. We see here that the large deviations behaviour of the solution of the Poissonian SDE and of its diffusion approximation differ dramatically, as was already noted by Pakdaman et al. [2010] (see also the references in this paper).

\section{Lower bound}

We first establish the LDP lower bound under the assumption that the rates are bounded away from zero, or in other words the $\log \beta_{j}$ 's are bounded. From this, we will derive later the general result.

\subsection{LDP lower bound if the rates are bounded away from zero}

We first note that if the $\beta_{j}$ are bounded away from zero, then the convex cone $\mathcal{C}_{x}$ is dependent of $x, \mathcal{C}_{x}=\mathcal{C}$ for all $x$. Note that this implies that the "domain" $A$ of the process cannot be bounded.

We require a LDP for linear functions. This follows from the LLN (Theorem 3.1). 
Proposition 5.1. Assume that $\log \beta_{j}(j=1, \ldots, k)$ is bounded and continuous. For any $\epsilon>0, \delta>0$ there exists an $\tilde{\epsilon}>0$ such that for $x \in A, y \in \mathcal{C}$ and $\mu \in V_{x, y}=\tilde{V}_{y}$,

$$
\liminf _{N \rightarrow \infty} \frac{1}{N} \log \left(\inf _{z \in A ;|z-x|<\tilde{\epsilon}} \mathbb{P}\left[\sup _{t \in[0, T]}\left|Z^{N, z}(t)-\phi^{x}(t)\right|<\epsilon\right]\right) \geq-\int_{0}^{T} \ell\left(\phi^{x}(t), \mu\right) d t-\delta,
$$

where

$$
\phi^{x}(t):=x+t y=x+t \sum_{j} \mu_{j} h_{j}
$$

Proof. We define

$$
F^{N, z}:=\left\{\sup _{t \in[0, T]}\left|Z^{N, z}(t)-\phi^{z}(t)\right|<\frac{\epsilon}{2}\right\}
$$

and let $\tilde{\epsilon}<\epsilon_{1}=\epsilon / 2$. Let now $\xi_{T}=\xi_{T}^{N, z}=\left.\frac{d \tilde{\mathbb{P}}}{d \mathbb{P}}\right|_{\mathcal{F}_{T}}$ be given as in Theorem A.1 for initial value $z$ and constant rates $\tilde{\beta}_{j}=\mu_{j}$. Then, with the notation $\tilde{\mathbb{E}}_{F^{N, z}}[X]:=\tilde{\mathbb{E}}\left[X \mid F^{N, z}\right]$ and (recall that $\xi_{T} \neq 0 \tilde{\mathbb{P}}$-almost surely)

$$
\begin{aligned}
X_{T}^{N, z}:=X_{T} & :=\log \xi_{T}^{-1}=\sum_{\tau \leq T}\left[\log \beta_{j(\tau)}\left(Z^{N, z}(\tau-)\right)-\log \mu_{j(\tau)}\right]-N \sum_{j} \int_{0}^{T}\left(\beta_{j}\left(Z^{N, z}(t)\right)-\mu_{j}\right) d t \\
\liminf _{N \rightarrow \infty} & \frac{1}{N} \log \left(\inf _{z \in A,|x-z|<\tilde{\epsilon}} \mathbb{P}\left[\sup _{t \in[0, T]}\left|Z^{N, z}(t)-\phi^{x}(t)\right|<\epsilon\right]\right) \\
& \geq \liminf _{N \rightarrow \infty} \frac{1}{N} \log _{z \in A,|x-z|<\tilde{\epsilon}} \inf \mathbb{P}\left[F^{N, z}\right] \\
& =\liminf _{N \rightarrow \infty} \frac{1}{N} \inf _{z \in A,|x-z|<\tilde{\epsilon}} \log \mathbb{P}\left[F^{N, z}\right] \\
& \geq \liminf _{N \rightarrow \infty} \frac{1}{N} \inf _{z \in A,|x-z|<\tilde{\epsilon}} \log \tilde{\mathbb{E}}\left[\xi_{T}^{-1} \mathbb{1}_{F^{N, z}}\right] \\
& =\liminf _{N \rightarrow \infty} \frac{1}{N} \inf _{z \in A,|x-z|<\tilde{\epsilon}} \log \left(\tilde{\mathbb{P}}\left[F^{N, z}\right] \tilde{\mathbb{E}}_{F^{N, z}}\left[\exp \left(X_{T}\right)\right]\right) \\
& \geq \liminf _{N \rightarrow \infty} \frac{1}{N} \inf _{z \in A,|x-z|<\tilde{\epsilon}} \log \tilde{\mathbb{P}}\left[F^{N, z}\right]+\liminf _{N \rightarrow \infty} \frac{1}{N} \quad \inf \tilde{i n f}_{z \in A,|x-z|<\tilde{\epsilon}} \log \tilde{\mathbb{E}}_{F^{N, z}}\left[\exp \left(X_{T}\right)\right] \\
& \geq \liminf _{N \rightarrow \infty} \inf _{z \in A,|x-z|<\tilde{\epsilon}} \tilde{\mathbb{E}}_{F^{N, z}}\left[\frac{X_{T}}{N}\right],
\end{aligned}
$$

where we have used Corollary $\mathrm{A.2}$ for the second inequality, Theorem 3.1 and Jensen's inequality on the last line. Note the independence of the constants $\tilde{C}_{1}, \tilde{C}_{2}$ of $z$ in Theorem 3.1 and hence

$$
\tilde{\mathbb{P}}\left[F^{N, z}\right] \rightarrow 1 \quad \text { as } N \rightarrow \infty \text { independently of } z
$$

We have

$$
\frac{1}{\tilde{\mathbb{P}}\left[F^{N, z}\right]} \tilde{\mathbb{E}}\left[\mathbb{1}_{F^{N, z}} T \sum_{j} \mu_{j}\right]=T \sum_{j} \mu_{j} .
$$


By the fact that the $\beta_{j}$ 's are bounded and continuous and by Theorem 3.1, we have for $j=1, \ldots, k$,

$$
\sup _{t \in[0, T]}\left|\beta_{j}\left(Z^{N, z}(t)\right)-\beta_{j}\left(\phi^{z}(t)\right)\right| \rightarrow 0 \quad \tilde{\mathbb{P}}-\text { a.s. }
$$

as $N \rightarrow \infty$ uniformly in $z$. This implies

$$
\frac{1}{\tilde{\mathbb{P}}\left[F^{N, z}\right]} \tilde{\mathbb{E}}\left[\mathbb{1}_{F^{N, z}} \int_{0}^{T} \sum_{j} \beta_{j}\left(Z^{N, z}(t)\right) d t\right] \longrightarrow \sum_{j} \int_{0}^{T} \beta_{j}\left(\phi^{z}(t)\right) d t
$$

as $N \rightarrow \infty$ uniformly in $z$.

Let us now define the following processes. For $z \in A, j=1, \ldots, k$ and $0 \leq t_{1}<t_{2} \leq T$ let

$$
Y_{j}^{N, z, t_{1}, t_{2}}:=\frac{1}{N} \cdot \# \text { jumps of } Z^{N, z} \text { in direction } h_{j} \text { in }\left[t_{1}, t_{2}\right] \text { 9 }
$$

Let furthermore $\tau_{j} \in[0, T]$ denote the jump times of $Z^{N}$ in direction $h_{j}$; we obtain

$$
\begin{aligned}
& \frac{1}{\tilde{\mathbb{P}}\left[F^{N, z}\right]} \sum_{j ; \mu_{j}>0} \tilde{\mathbb{E}}\left[\frac{1}{N} \mathbb{1}_{F^{N, z}} \sum_{\tau_{j}} \log \mu_{j}\right] \\
& =\frac{1}{\tilde{\mathbb{P}}\left[F^{N, z}\right]} \sum_{j ; \mu_{j}>0} \log \mu_{j}\left\{\tilde{\mathbb{E}}\left[Y_{j}^{N, z, 0, T}\right] \tilde{\mathbb{P}}\left[F^{N, z}\right]+\widetilde{\operatorname{Cov}}\left(\mathbb{1}_{F^{N, z}}, Y_{j}^{N, z, 0, T}\right)\right\} \\
& \quad \rightarrow T \sum_{j} \mu_{j} \log \mu_{j},
\end{aligned}
$$

since, for a given set $F$,

$$
\begin{aligned}
\tilde{\mathbb{E}}\left[Y_{j}^{N, z, t_{1}, t_{2}}\right] & =\left(t_{2}-t_{1}\right) \mu_{j} \\
\widetilde{\operatorname{Var}}\left[Y_{j}^{N, z, t_{1}, t_{2}}\right] & =\left(t_{2}-t_{1}\right) \mu_{j} \\
\left|\widetilde{\operatorname{Cov}}\left(\mathbb{1}_{F}, Y_{j}^{N, z, t_{1}, t_{2}}\right)\right| & \leq \sqrt{\widetilde{\operatorname{Var}}\left[\mathbb{1}_{F}\right]} \sqrt{\widetilde{\operatorname{Var}}\left[Y_{j}^{N, z, t_{1}, t_{2}}\right]}=\sqrt{\tilde{\mathbb{P}}[F]-\tilde{\mathbb{P}}[F]^{2}} \sqrt{\left(t_{2}-t_{1}\right) \mu_{j}} .
\end{aligned}
$$

We now define the set

$$
\tilde{F}^{N, z}:=\left\{\sup _{t \in[0, T]}\left|Z^{N, z}(t)-\phi^{z}(t)\right|<\epsilon_{N}\right\} \quad \text { for } \epsilon_{N}:=\epsilon \wedge \frac{1}{N^{1 / 3}} ;
$$

we have (for $N$ large enough)

$$
\tilde{\mathbb{P}}\left[\tilde{F}^{N, z}\right] \geq 1-\tilde{C}_{1} \exp \left(-N \tilde{C}_{2}\left(\epsilon_{N}\right)\right) \longrightarrow 1
$$

as $N \rightarrow \infty$ uniformly in $z$ by Theorem 3.1. We furthermore let $\bar{A} \subset A$ be compact such that for all $z,|z-x|<\tilde{\epsilon}$ and all $t \in[0, T], \phi^{z}(t) \in \bar{A}$ and $Z^{N, z}(t) \in \bar{A}$ on $F^{N, z}$. As the $\log \beta_{j}$ are bounded and uniformly continuous, there exit constants $\tilde{\delta}_{N}>0$ with $\tilde{\delta}_{N} \downarrow 0$ such that

$$
\tilde{x}, \bar{x} \in \bar{A},|\tilde{x}-\bar{x}|<\frac{2}{N^{1 / 3}} \Rightarrow\left|\log \beta_{j}(\tilde{x})-\log \beta_{j}(\bar{x})\right|<\tilde{\delta}_{N}
$$

We define $\bar{\mu}=\max _{j} \mu_{j}$,

$$
M=M(N):=\left\lfloor T N^{1 / 3} k \bar{h} \bar{\mu}+1\right\rfloor
$$


and divide the interval $[0, T]$ into $M$ subintervals $\left[t_{r}, t_{r+1}\right]\left(r=0, \ldots, M-1, t_{r}=t_{r}(N)\right)$ of length $\Delta=\Delta(N)$, i.e. for $N \geq N_{0}$ independent of $z$ large enough,

$$
\Delta<\frac{1}{N^{1 / 3} k \bar{\mu} \bar{h}}
$$

For $j, r=0, \ldots, M-1$ and $\tau_{j}, t \in\left[t_{r}, t_{r+1}\right]$, since for $|t-s|<\frac{1}{N^{1 / 3} k \bar{\mu} h},\left|\phi^{z}(t)-\phi^{z}(s)\right|<\frac{1}{N^{1 / 3}}$, we have on $\tilde{F}^{N, z}$

$$
\left|Z^{N, z}\left(\tau_{j}-\right)-\phi^{z}(t)\right| \leq\left|Z^{N, z}\left(\tau_{j}-\right)-\phi^{z}\left(\tau_{j}\right)\right|+\left|\phi^{z}\left(\tau_{j}\right)-\phi^{z}(t)\right| \leq \frac{2}{N^{1 / 3}},
$$

and hence

$$
\inf _{t \in\left[t_{r}, t_{r+1}\right]} \log \beta_{j}\left(\phi^{z}(t)\right)-\tilde{\delta}_{N} \leq \log \beta_{j}\left(Z^{N, z}\left(\tau_{j}-\right)\right) \leq \sup _{t \in\left[t_{r}, t_{r+1}\right]} \log \beta_{j}\left(\phi^{z}(t)\right)+\tilde{\delta}_{N} .
$$

We compute

$$
\begin{aligned}
& \frac{1}{\tilde{\mathbb{P}}\left[F^{N, z}\right]} \tilde{\mathbb{E}}\left[\frac{1}{N} \mathbb{1}_{F^{N, z}} \sum_{\tau} \log \beta_{j(\tau)}\left(Z^{N, z}(\tau-)\right)\right] \\
& =\frac{1}{\tilde{\mathbb{P}}\left[F^{N, z}\right]} \sum_{j, \mu_{j}>0} \sum_{r=0}^{M-1} \tilde{\mathbb{E}}\left[\frac{1}{N} \mathbb{1}_{\tilde{F}^{N, z}} \sum_{\tau_{j} \in\left[t_{r}, t_{r+1}\right)} \log \beta_{j}\left(Z^{N, z}\left(\tau_{j}-\right)\right)\right] \\
& +\frac{1}{\tilde{\mathbb{P}}\left[F^{N, z}\right]} \sum_{j, \mu_{j}>0} \sum_{r=0}^{M-1} \tilde{\mathbb{E}}\left[\frac{1}{N} \mathbb{1}_{F^{N, z} \backslash \tilde{F}^{N, z}} \sum_{\tau_{j} \in\left[t_{r}, t_{r+1}\right)} \log \beta_{j}\left(Z^{N, z}\left(\tau_{j}-\right)\right)\right] \\
& \leq \frac{1}{\tilde{\mathbb{P}}\left[F^{N, z}\right]} \sum_{j, \mu_{j}>0} \sum_{r=0}^{M-1}\left(\sup _{t \in\left[t_{r}, t_{r+1}\right)} \log \beta_{j}\left(\phi^{z}(t)\right)+\tilde{\delta}_{N}\right) \tilde{\mathbb{E}}\left[\mathbb{1}_{\tilde{F}^{N, z}} Y_{j}^{N, z, t_{r}, t_{r+1}}\right] \\
& +\frac{1}{\tilde{\mathbb{P}}\left[F^{N, z}\right]} \sum_{j, \mu_{j}>0} \sum_{r=0}^{M-1} \log \bar{\beta} \tilde{\mathbb{E}}\left[\mathbb{1}_{F^{N, z} \backslash \tilde{F}^{N, z}} Y_{j}^{N, z, t_{r}, t_{r+1}}\right] \\
& \leq \frac{1}{\tilde{\mathbb{P}}\left[F^{N, z}\right]} \sum_{j, \mu_{j}>0} \sum_{r=0}^{M-1} \sup _{t \in\left[t_{r}, t_{r+1}\right)} \log \beta_{j}\left(\phi^{z}(t)\right)\left\{\tilde{\mathbb{E}}\left[\mathbb{1}_{\tilde{F}^{N, z}}\right] \tilde{\mathbb{E}}\left[Y_{j}^{N, z, t_{r}, t_{r+1}}\right]+\widetilde{\operatorname{Cov}}\left(\mathbb{1}_{\tilde{F}^{N, z}}, Y_{j}^{N, z, t_{r}, t_{r+1}}\right)\right\} \\
& +\frac{1}{\tilde{\mathbb{P}}\left[F^{N, z}\right]} \sum_{j, \mu_{j}>0} \sum_{r=0}^{M-1} \log \bar{\beta}\left\{\tilde{\mathbb{E}}\left[\mathbb{1}_{F^{N, z} \backslash \tilde{F}^{N, z}}\right] \tilde{\mathbb{E}}\left[Y_{j}^{N, z, t_{r}, t_{r+1}}\right]+\widetilde{\operatorname{Cov}}\left(\mathbb{1}_{F^{N, z} \backslash \tilde{F}^{N, z}}, Y_{j}^{N, z, t_{r}, t_{r+1}}\right)\right\} \\
& +\frac{1}{\tilde{\mathbb{P}}\left[F^{N, z}\right]} \tilde{\delta}_{N} k \bar{\mu} T \\
& \leq \frac{\tilde{\mathbb{P}}\left[\tilde{F}^{N, z}\right]}{\tilde{\mathbb{P}}\left[F^{N, z}\right]} \sum_{j, \mu_{j}>0} \mu_{j} \sum_{r=0}^{M-1} \Delta \sup _{t \in\left[t_{r}, t_{r+1}\right)} \log \beta_{j}\left(\phi^{z}(t)\right) \\
& +\frac{1}{\tilde{\mathbb{P}}\left[F^{N, z}\right]}\left\{\log \bar{\beta} \sum_{j, \mu_{j}>0} \sum_{r=0}^{M-1}\left(\left|\widetilde{\operatorname{Cov}}\left(\mathbb{1}_{\tilde{F}^{N, z}}, Y_{j}^{N, z, t_{r}, t_{r+1}}\right)\right|+\left|\widetilde{\operatorname{Cov}}\left(\mathbb{1}_{F^{N, z} \backslash \tilde{F}^{N, z}}, Y_{j}^{N, z, t_{r}, t_{r+1}}\right)\right|\right)\right. \\
& \left.+\tilde{\delta}_{N} k \bar{\mu} T+\tilde{\mathbb{P}}\left[F^{N, z} \backslash \tilde{F}^{N, z}\right] k \bar{\mu} T \log \bar{\beta}\right\}
\end{aligned}
$$




$$
=: \bar{S}^{N, z}+\bar{U}^{N, z} \text {, }
$$

where $\bar{S}^{N, z}$ and $\bar{U}^{N, z}$ are the first respectively the second term in Inequality (35). In a similar fashion we obtain

$$
\begin{aligned}
& \frac{1}{\tilde{\mathbb{P}}\left[F^{N, z}\right]} \tilde{\mathbb{E}}\left[\frac{1}{N} \mathbb{1}_{F^{N, z}} \sum_{\tau} \log \beta_{j(\tau)}\left(Z^{N, z}(\tau-)\right)\right] \\
& \geq \frac{\tilde{\mathbb{P}}\left[\tilde{F}^{N, z}\right]}{\tilde{\mathbb{P}}\left[F^{N, z}\right]} \sum_{j, \mu_{j}>0} \mu_{j} \sum_{r=0}^{M-1} \Delta \inf _{t \in\left[t_{r}, t_{r+1}\right)} \log \beta_{j}\left(\phi^{z}(t)\right) \\
& \quad+\frac{1}{\tilde{\mathbb{P}}\left[F^{N, z}\right]}\left\{\log \underline{\beta} \sum_{j, \mu_{j}>0} \sum_{r=0}^{M-1}\left(\widetilde{\operatorname{Cov}}\left(\mathbb{1}_{\tilde{F}^{N, z}}, Y_{j}^{N, z, t_{r}, t_{r+1}}\right)|+| \widetilde{\operatorname{Cov}}\left(\mathbb{1}_{F^{N, z} \backslash \tilde{F}^{N, z}}, Y_{j}^{N, z, t_{r}, t_{r+1}}\right) \mid\right)\right. \\
&\left.\quad-\tilde{\delta}_{N} k \bar{\mu} T+\tilde{\mathbb{P}}\left[F^{N, z} \backslash \tilde{F}^{N, z}\right] k \bar{\mu} T \log \underline{\beta}\right\} \\
&=: \underline{S}^{N, z}+\underline{U}^{N, z}
\end{aligned}
$$

we first note that $\bar{U}^{N, z}, \underline{U}^{N, z} \rightarrow 0$ as $N \rightarrow \infty$ uniformly in $z$, since $\tilde{\delta}_{N} \rightarrow 0$ and $\tilde{\mathbb{P}}\left[F^{N, z} \backslash\right.$ $\left.\tilde{F}^{N, z}\right] \rightarrow 0$ as $N \rightarrow 0$ uniformly in $z$. Furthermore, as (up to a factor which converges to 1 uniformly in $z) \bar{S}^{N, z}$ and $\underline{S}^{N, z}$ are upper respectively lower Riemann sums, we obtain

$$
\bar{S}^{N, z}, \underline{S}^{N, z} \rightarrow \sum_{j} \mu_{j} \int_{0}^{T} \log \beta_{j}\left(\phi^{z}(t)\right) d t
$$

as $N \rightarrow \infty$; since

$$
\left|\bar{S}^{N, z}-\underline{S}^{N, z}\right| \leq 2 \frac{\tilde{\mathbb{P}}\left[\tilde{F}^{N, z}\right]}{\tilde{\mathbb{P}}\left[F^{N, z}\right]} k \bar{\mu} \tilde{\delta}_{N} \rightarrow 0
$$

uniformly in $z$, the convergence in (36) is likewise uniform in $z$.

The uniform convergence implies (cf. (31) - (34) and the preceding discussion) that

$$
\liminf _{N \rightarrow \infty} \frac{1}{N} \log \left(\inf _{z \in A,|x-z|<\tilde{\epsilon}} \mathbb{P}\left[\sup _{t \in[0, T]}\left|Z^{N, z}(t)-\phi^{x}(t)\right|<\epsilon\right]\right) \geq-\sup _{z \in A,|x-z|<\tilde{\epsilon}} \int_{0}^{T} \ell\left(\phi^{z}(t), \mu\right) d t
$$

In combination with the uniform continuity of $\ell(\cdot, \mu)$ (recall the boundedness of the $\log \beta_{j}$ ) this proves the assertion.

The main building block for the lower bound is the following result.

Theorem 5.2. Assume that $\log \beta_{j}(j=1, \ldots, k)$ is bounded and continuous. Let $\phi \in$ $D([0, T] ; A)$ with $\phi(0)=x$ and $\epsilon>0$. Then,

$$
\liminf _{N \rightarrow \infty} \frac{1}{N} \log \mathbb{P}\left[\sup _{t \in[0, T]}\left|Z^{N, x}(t)-\phi(t)\right|<\epsilon\right] \geq-I_{T, x}(\phi) .
$$

The convergence is uniform in $x \in A$. 
Proof. We can w.l.o.g. assume that $I_{T, x}(\phi)<\infty$ (and hence $\phi$ is absolutely continuous) as else the assertion is trivial. We approximate the function $\phi$ by a continuous piecewise linear function and then apply the LDP for linear functions to each of these linear functions. To this end, we let $\delta>0$ and divide the interval $[0, T]$ into $J$ subintervals of length $\Delta=T / J,\left[t_{r-1}, t_{r}\right](r=1, \ldots, J)$ such that the resulting piecewise linear approximation

$$
\tilde{\phi}(t)=\phi\left(t_{r-1}\right)+\frac{t-t_{r-1}}{\Delta}\left(\phi\left(t_{r}\right)-\phi\left(t_{r-1}\right)\right)
$$

satisfies

$$
\sup _{t \in[0, T]}|\phi(t)-\tilde{\phi}(t)|<\frac{\epsilon}{2}
$$

(recall that $\phi$ is continuous).

We now apply Theorem 4.18 twice (in Inequalities (37) and (39)) and choose $J$ large enough in order to assure

$$
\begin{aligned}
\int_{0}^{T} L\left(\phi(t), \phi^{\prime}(t)\right) d t & =\sum_{r=1}^{J} \int_{t_{r-1}}^{t_{r}} L\left(\phi(t), \phi^{\prime}(t)\right) d t \\
& \geq \sum_{r=1}^{J} \int_{t_{r-1}}^{t_{r}} L\left(\phi\left(t_{r-1}\right), \phi^{\prime}(t)\right) d t-\frac{\delta}{4} \\
& \geq \Delta \sum_{r=1}^{J} L\left(\phi\left(t_{r-1}\right), \frac{\Delta \phi\left(t_{r}\right)}{\Delta}\right)-\frac{\delta}{4} \\
& \geq \sum_{r=1}^{J} \int_{t_{r-1}}^{t_{r}} L\left(\tilde{\phi}(t), \tilde{\phi}^{\prime}(t)\right) d t-\frac{\delta}{2},
\end{aligned}
$$

where

$$
\Delta \phi\left(t_{r}\right):=\phi\left(t_{r}\right)-\phi\left(t_{r-1}\right)
$$

Note that for Inequality (38), we have applied Jensen's inequality and the fact that $L$ is convex in its second argument (cf. Corollary 4.10). As $I_{T, x}(\tilde{\phi})<\infty$, this implies

$$
\Delta \phi\left(t_{r}\right) \in \mathcal{C} \text { for all } r \text {. }
$$

We note that by the continuity of $L(\cdot, y), \mu^{*}(x, y)$ (the minimizing $\mu \in V_{x, y}=\tilde{V}_{y}$ for $\ell(x, \cdot))$ is "almost optimal" for all $\tilde{x}$ sufficiently close to $x$ (in the sense that $\ell\left(\tilde{x}, \mu^{*}(x, y)\right.$ ) is close to $L(\tilde{x}, y))$. By dividing each interval $\left[t_{r-1}, t_{r}\right]$ into further subintervals $\left[s_{j-1}, s_{j}\right]$ if necessary, we can hence represent the directions $\Delta \phi\left(t_{k}\right) / \Delta$ by

$$
\mu^{j} \in V_{\tilde{\phi}(t), \Delta \phi\left(t_{r}\right) / \Delta}=\tilde{V}_{\Delta \phi\left(t_{r}\right) / \Delta}
$$

in such a way that

$$
L\left(\tilde{\phi}(t), \frac{\Delta \phi\left(t_{r}\right)}{\Delta}\right) \geq \ell\left(\tilde{\phi}(t), \mu^{j}\right)-\frac{\delta}{4 T} \quad \text { for all } t \in\left[s_{j-1}, s_{j}\right] .
$$

For simplicity of exposition, we assume that this further subdivision of the intervals $\left[t_{r-1}, t_{r}\right]$ is not required and denote the "almost optimal" $\mu$ 's by $\mu^{r}(r=1, \ldots, J)$. Hence

$$
\int_{0}^{T} L\left(\phi(t), \phi^{\prime}(t)\right) d t \geq \sum_{r=1}^{J} \int_{t_{r-1}}^{t_{r}} \ell\left(\tilde{\phi}(t), \mu^{r}\right)-\frac{3 \delta}{4} .
$$


Choose now $\tilde{\epsilon}=\tilde{\epsilon}_{J-1}$ according to Proposition 5.1 corresponding to $\epsilon / 2, \delta /(4 J)$, initial value $\tilde{\phi}\left(t_{J-1}\right)$ and time-horizon $\Delta$. Using the Markov property of $Z^{N}$, we compute

$$
\begin{aligned}
\mathbb{P}\left[\sup _{t \in[0, T]}\left|Z^{N, x}(t)-\phi(t)\right|<\epsilon\right] \geq & \mathbb{P}\left[\sup _{t \in\left[0, t_{J-1}\right]}\left|Z^{N, x}(t)-\tilde{\phi}(t)\right|<\tilde{\epsilon}\right] \\
& \quad \inf _{z \in A ;\left|z-\tilde{\phi}\left(t_{J-1}\right)\right|<\tilde{\epsilon}} \mathbb{P}\left[\sup _{t \in\left[t_{J-1}, T\right]}\left|Z^{N, z}(t)-\tilde{\phi}(t)\right|<\frac{\epsilon}{2}\right]
\end{aligned}
$$

here, we denote (by a slight abuse of notation) the process starting at $z$ at time $t_{J-1}$ by $Z^{N, z}$. Proposition 5.1 implies

$$
\begin{aligned}
\liminf _{N \rightarrow \infty} & \frac{1}{N} \log \mathbb{P}\left[\sup _{t \in[0, T]}\left|Z^{N, x}(t)-\phi(t)\right|<\epsilon\right] \\
\geq & \liminf _{N \rightarrow \infty} \frac{1}{N} \log \mathbb{P}\left[\sup _{t \in\left[0, t_{J-1}\right]}\left|Z^{N, x}(t)-\tilde{\phi}(t)\right|<\tilde{\epsilon}_{J-1}\right] \\
& \quad+\liminf _{N \rightarrow \infty} \frac{1}{N} \log \left(\inf _{z \in A ;\left|z-\tilde{\phi}\left(t_{J-1}\right)\right|<\tilde{\epsilon}} \mathbb{P}\left[\sup _{t \in\left[t_{J-1}, T\right]}\left|Z^{N, z}(t)-\tilde{\phi}(t)\right|<\frac{\epsilon}{2}\right]\right) \\
\geq & \liminf _{N \rightarrow \infty} \frac{1}{N} \log \mathbb{P}\left[\sup _{t \in\left[0, t_{J-1}\right]}\left|Z^{N, x}(t)-\tilde{\phi}(t)\right|<\tilde{\epsilon}_{J-1}\right]-\int_{t_{J-1}}^{T} \ell\left(\tilde{\phi}(t), \mu^{J}\right) d t-\frac{\delta}{4 J} .
\end{aligned}
$$

Iterating this procedure, we obtain

$$
\liminf _{N \rightarrow \infty} \frac{1}{N} \log \mathbb{P}\left[\sup _{t \in[0, T]}\left|Z^{N, x}(t)-\phi(t)\right|<\epsilon\right] \geq-\sum_{r=1}^{J} \int_{t_{r-1}}^{t_{r}} \ell\left(\tilde{\phi}(t), \mu^{r}\right) d t-\frac{\delta}{4}
$$

and the assertion follows from Inequality (40) if we let $\delta \rightarrow 0$.

We note that the convergence is uniform in $x$ by the uniformity in Proposition 5.1 .

Theorem 5.3. Assume that $\log \beta_{j}(j=1, \ldots, k)$ is bounded and continuous. Let $G \subset$ $D([0, T] ; A)$ be open and $x \in A$. Then,

$$
\liminf _{N \rightarrow \infty} \frac{1}{N} \log \mathbb{P}\left[Z^{N, x} \in G\right] \geq-\inf _{\phi \in G,} I_{T, x}(\phi) .
$$

The convergence is uniform in $x \in A$.

Proof. Let $\inf _{\phi \in G} I_{T, x}(\phi)=: I^{*}<\infty$; hence, for $\delta>0$, there exists a $\phi^{\delta} \in G(\phi(0)=x)$ with $I_{T, x}\left(\phi^{\delta}\right) \leq I^{*}+\delta$. For small enough $\epsilon=\epsilon\left(\phi^{\delta}\right)>0$, we have

$$
\left\{\phi \in D([0, T] ; A)\left|\sup _{t \in[0, T]} ;\right| \phi^{\delta}(t)-\phi(t) \mid<\epsilon\right\} \subset G
$$

and therefore

$$
\mathbb{P}\left[\sup _{t \in[0, T]}\left|Z^{N, x}(t)-\phi^{\delta}(t)\right|<\epsilon\right] \leq \mathbb{P}\left[Z^{N, x} \in G\right] .
$$

This implies by Theorem 5.2 that for all $\delta>0$,

$$
\liminf _{N \rightarrow \infty} \frac{1}{N} \log \mathbb{P}\left[Z^{N, x} \in G\right] \geq \liminf _{N \rightarrow \infty} \frac{1}{N} \log \mathbb{P}\left[\sup _{t \in[0, T]}\left|Z^{N, x}(t)-\phi^{\delta}(t)\right|<\epsilon\right]
$$




$$
\begin{aligned}
& \geq-I_{T, x}\left(\phi^{\delta}\right) \\
& \geq-I^{*}-\delta .
\end{aligned}
$$

This implies

$$
\liminf _{N \rightarrow \infty} \frac{1}{N} \log \mathbb{P}\left[Z^{N, x} \in G\right] \geq-I^{*}
$$

as desired.

We obtain the following result.

Corollary 5.4. Assume that $\log \beta_{j}(j=1, \ldots, k)$ is bounded and continuous. Then for all $\phi \in D([0, T] ; A)$ with $\phi(0)=x$ and $\epsilon, \delta>0$, there exists an $\tilde{\epsilon}>0$ such that

$$
\liminf _{N \rightarrow \infty} \frac{1}{N} \log \left(\inf _{z \in A ;|x-z|<\tilde{\epsilon}} \mathbb{P}\left[\sup _{t \in[0, T]}\left|Z^{N, z}-\phi(t)\right|<\epsilon\right]\right) \geq-I_{T, x}(\phi)-\delta .
$$

Proof. We assume w.l.o.g. that $I_{T, x}(\phi)<\infty$. By Theorem 5.3, there exists an $N_{0}$ and $\tilde{\epsilon}$ such that for $N \geq N_{0}$ and $z$ with $|z-x|<\tilde{\epsilon}$,

$$
\frac{1}{N} \log \mathbb{P}\left[\sup _{t \in[0, T]}\left|Z^{N, z}-\phi(t)\right|<\epsilon\right] \geq-\inf _{\tilde{\phi}:\|\phi-\tilde{\phi}\|<\epsilon} I_{T, x}(\tilde{\phi})-\delta \geq-I_{T, x}(\phi)-\delta .
$$

The assertion follows.

\subsection{LDP lower bound with vanishing rates}

In the following, we drop the assumption that the log-rates are bounded. Instead, we rather consider situations, where Assumption 2.3 is satisfied.

We start by some preliminary considerations and assume that Assumption 2.3 (A1) and (A2) are satisfied. We note that there exists a constant $\alpha>0$ such that for all $x \in A$ there exists a $i \leq I$ such that $B(x, \alpha) \subset B_{i}$. Indeed, assume that this is incorrect and consider a sequence of points $x_{n} \in A$ such that $B\left(x_{n}, 1 / n\right)$ is not contained in any $B_{i}$. W.l.o.g., we can assume that $x_{n} \rightarrow x \in A$ (recall that $A$ is compact). As $x \in B_{i_{0}}$ for some $i_{0}$, we have $B\left(x_{n}, 1 / n\right) \subset B_{i_{0}}$ for $n$ large enough, a contradiction.

Lemma 5.5. Assume that $\beta_{j}(j=1, \ldots, k)$ is bounded and that Assumption 2.3 (A1) and (A2) are satisfied. Then, for $T>0, K>0$, there exists a $J=J(T, K) \in \mathbb{N}$ such that for all $\phi \in D([0, T] ; A)$ with $I_{T}(\phi) \leq K$, there exist

$$
0=t_{0}<t_{1}<\cdots<t_{J}=T \text { and } i_{1}, \ldots, i_{J} \text { such that } \phi(t) \in B_{i_{r}} \text { for } t \in\left[t_{r-1}, t_{r}\right] \text {. }
$$

Furthermore, for $r=1, \ldots, J$,

$$
\operatorname{dist}\left(\phi\left(t_{r-1}\right), \partial B_{i_{r}}\right) \geq \alpha \quad \text { and } \quad \operatorname{dist}\left(\phi(t), \partial B_{i_{r}}\right) \geq \alpha / 2 \quad \text { for } t \in\left[t_{r-1}, t_{r}\right]
$$

for $\alpha$ as before. 
Proof. By the considerations above, we have $B(x, \alpha) \subset B_{i_{1}}$ for an appropriate $i_{1}$. We define

$$
\tilde{t}_{1}:=\inf \left\{t \geq 0 \mid B(\phi(t), \alpha / 2) \not \subset B_{i_{1}}\right\} \wedge T>0 .
$$

Now, there exists an $i_{2}$ such that $B\left(\phi\left(t_{1}\right), \alpha\right) \subset B_{i_{2}}$. If $\tilde{t}_{1}<T$, we define

$$
\tilde{t}_{2}:=\inf \left\{t \geq t_{1} \mid B(\phi(t), \alpha / 2) \not \subset B_{i_{2}}\right\} \wedge T>\tilde{t}_{1} .
$$

In the same way, we proceed. By the uniform absolute continuity (Lemma 4.17) of all $\phi$ with $I_{T}(\phi) \leq K$, we have

$$
\tilde{t}_{r}-\tilde{t}_{r-1} \geq \delta \text { for a constant } \delta>0 \text { independent of } \phi .
$$

The assertion hence follows for $J:=\left\lfloor\frac{T}{\delta}\right\rfloor+1$ and $t_{r}:=r \delta(r=1, \ldots, J-1), t_{J}:=T$.

We now define a function $\phi^{\eta}$ which is close to a given function $\phi$ with $I_{T}(\phi)<\infty$. We assume that Assumption 2.3 (A) holds. Hence, for $x \in B_{i} \cap A$ and $t \in\left(0, \lambda_{2}\right)$,

$$
\mathrm{d}\left(x+t v_{i}, \partial A\right)>\lambda_{1} t .
$$

Note that $\lambda_{1} \leq 1$. Let $\eta>0$ be small. We define for $r=1, \ldots, J$, with the notation $\sum_{j=1}^{0} \ldots=0$,

$$
\eta_{r}:=\eta \sum_{j=1}^{r}\left(\frac{3}{\lambda_{1}}\right)^{j-1}
$$

- For $r=1, \ldots, J, t \in\left[t_{r-1}+\eta_{r-1}, t_{r-1}+\eta_{r}\right]$,

$$
\phi^{\eta}(t):=\phi\left(t_{r-1}\right)+\eta \sum_{j=1}^{r-1}\left(\frac{3}{\lambda_{1}}\right)^{j-1} v_{i_{j}}+\left(t-t_{r-1}-\eta_{r-1}\right) v_{i_{r}}
$$

- For $r=1, \ldots, J, t \in\left[t_{r-1}+\eta_{r}, t_{r}+\eta_{r}\right]$,

$$
\phi^{\eta}(t):=\phi\left(t-\eta_{r}\right)+\eta \sum_{j=1}^{r}\left(\frac{3}{\lambda_{1}}\right)^{j-1} v_{i_{j}} .
$$

We make the following assumptions on $\eta$ :

$$
\eta_{J}=\eta \sum_{r=1}^{J}\left(\frac{3}{\lambda_{1}}\right)^{r-1} \leq \frac{\alpha}{4} \wedge \min _{r=1, \ldots, J}\left|t_{r}-t_{r-1}\right| \wedge \lambda_{2} .
$$

Therefore, we have the following properties for $\phi^{\eta}$ :

- For $r=1, \ldots, J, t \in\left[t_{r-1}+\eta_{r-1}, t_{r-1}+\eta_{r}\right]$,

$$
\left|\phi(t)-\phi^{\eta}(t)\right| \leq\left|\phi(t)-\phi\left(t_{r-1}\right)\right|+\eta_{r-1}+\left(\eta_{r}-\eta_{r-1}\right)=V_{t_{r}-t_{r-1}}(\phi)+\eta_{r} \rightarrow 0
$$

as $\eta \rightarrow 0$, where $V$. $(\phi)$ is the modulus of continuity of $\phi$. Similarly, for $r=1, \ldots, J$, $t \in\left[t_{r-1}+\eta_{r}, t_{r}+\eta_{r}\right]$,

$$
\left|\phi(t)-\phi^{\eta}(t)\right| \leq\left|\phi(t)-\phi\left(t-\eta_{r}\right)\right|+\eta_{r}=V_{\eta_{r}}(\phi)+\eta_{r} \rightarrow 0
$$

as $\eta \rightarrow 0$. 
- For $r=1, \ldots, J, t \in\left[t_{r-1}+\eta_{r-1}, t_{r-1}+\eta_{r}\right]$,

$$
\operatorname{dist}\left(\phi^{\eta}(t), \partial B_{i_{r}}\right) \geq \operatorname{dist}\left(\phi\left(t_{r-1}\right), \partial B_{i_{r}}\right)-\eta_{r} \geq \alpha-\frac{\alpha}{4} .
$$

Similarly, for $r=1, \ldots, J, t \in\left[t_{r-1}+\eta_{r}, t_{r}+\eta_{r}\right]$, hence $t-\eta_{r} \in\left[t_{r-1}, t_{r}\right]$,

$$
\operatorname{dist}\left(\phi^{\eta}(t), \partial B_{i_{r}}\right) \geq \operatorname{dist}\left(\phi\left(t-\eta_{r}\right), \partial B_{i_{r}}\right)-\eta_{r} \geq \frac{\alpha}{2}-\frac{\alpha}{4} .
$$

Hence, for $r=1, \ldots, J, t \in\left[t_{r-1}+\eta_{r-1}, t_{r}+\eta_{r}\right]$,

$$
\operatorname{dist}\left(\phi^{\eta}(t), \partial B_{i_{r}}\right) \geq \frac{\alpha}{4} \text {. }
$$

- For $t \in[0, \eta]$,

$$
\operatorname{dist}\left(\phi^{\eta}(t), \partial A\right) \geq t \lambda_{1}
$$

For $t \in\left[\eta, T+\eta_{J}\right]$,

$$
\operatorname{dist}\left(\phi^{\eta}(t), \partial A\right) \geq \lambda_{1} \eta
$$

This can be seen by induction on $r=1, \ldots, J$ (the induction hypothesis is clear, cf. Inequality (42) ). For $r=1, \ldots, J$, we have (by induction hypothesis and the assumptions on $\eta$ )

$$
\phi^{\eta}\left(t_{r-1}+\eta_{r-1}\right) \in B_{i_{r}}, \text { and for } r \geq 2, \operatorname{dist}\left(\phi^{\eta}\left(t_{r-1}+\eta_{r-1}\right), \partial A\right) \geq \eta \lambda_{1} .
$$

From Assumption 2.3 (A3), the distance of $\phi^{\eta}(t)$ to the boundary is increasing for $t \in\left[t_{r-1}+\eta \sum_{j=1}^{r-1}\left(\frac{3}{\lambda_{1}}\right)^{j-1}, t_{r-1}+\eta \sum_{j=1}^{r}\left(\frac{3}{\lambda_{1}}\right)^{j-1}\right]$, and is at least

$$
\left(t-\left(t_{r-1}+\eta \sum_{j=1}^{r-1}\left(\frac{3}{\lambda_{1}}\right)^{j-1}\right)\right) \cdot \lambda_{1} \vee \eta \lambda_{1} \text {. }
$$

In particular,

$$
\operatorname{dist}\left(\phi^{\eta}\left(t_{r-1}+\eta \sum_{j=1}^{r}\left(\frac{3}{\lambda_{1}}\right)^{j-1}\right), \partial A\right) \geq \eta \lambda_{1}\left(\frac{3}{\lambda_{1}}\right)^{r-1} \text {. }
$$

For $t \in\left[t_{r-1}+\eta \sum_{j=1}^{r}\left(\frac{3}{\lambda_{1}}\right)^{j-1}, t_{r}+\eta \sum_{j=1}^{r}\left(\frac{3}{\lambda_{1}}\right)^{j-1}\right]$, we have

$$
\begin{aligned}
\phi^{\eta}(t) & =\phi\left(t-\eta \sum_{j=1}^{r}\left(\frac{3}{\lambda_{1}}\right)^{j-1}\right)+\eta\left(\frac{3}{\lambda_{1}}\right)^{r-1} v_{i_{r}}+\eta \sum_{j=1}^{r-1}\left(\frac{3}{\lambda_{1}}\right)^{j-1} v_{i_{j}} \\
& =\bar{\phi}(t)+\eta \sum_{j=1}^{r-1}\left(\frac{3}{\lambda_{1}}\right)^{j-1} v_{i_{j}}
\end{aligned}
$$

and therefore (by elementary calculus and the fact that $\left|v_{i}\right| \leq 1$ )

$$
\begin{aligned}
\operatorname{dist}\left(\phi^{\eta}(t), \partial A\right) & \geq \operatorname{dist}(\bar{\phi}(t), \partial A)-\left|\eta \sum_{j=1}^{r-1}\left(\frac{3}{\lambda_{1}}\right)^{j-1} v_{i_{j}}\right| \\
& \geq \eta\left(\frac{3}{\lambda_{1}}\right)^{r-1} \lambda_{1}\left(1-\frac{\mathbf{1}_{r \geq 2}}{2}\right) \\
& \geq \eta \lambda_{1} .
\end{aligned}
$$


We now have

Lemma 5.6. Assume that Assumption 2.3 holds. Let $K>0$ and $\epsilon>0$. Then there exists an $\eta_{0}=\eta_{0}(T, K, \epsilon)>0$ such that for all $\phi \in D([0, T] ; A)$ with $I_{T}(\phi) \leq K$ and all $\eta<\eta_{0}$,

$$
I_{T}\left(\phi^{\eta}\right) \leq I_{T}(\phi)+\epsilon
$$

where $\phi^{\eta}(t)$ is defined as above.

Proof. We first use Lemma 2.2 and chose $\eta<\eta_{1}$ small enough (independent of $i, \phi$ ) such that

$$
\sum_{r=1}^{J} \int_{t_{r-1}+\eta_{r-1}}^{t_{r-1}+\eta_{r}} L\left(\phi^{\eta}(t),\left(\phi^{\eta}\right)^{\prime}(t)\right) d t=\sum_{r=1}^{J} I_{\eta_{r}-\eta_{r-1}}(\phi)<\frac{\epsilon}{2}
$$

We now denote by $\mu^{*}(t)$ the optimal $\mu$ corresponding to $\left(\phi(t), \phi^{\prime}(t)\right)$ (cf. Lemma 4.5). We let $r=1, \ldots, J$ and $t \in\left[t_{r-1}+\eta_{r}, t_{r}+\eta_{r}\right]$ and note that $\left(\phi^{\eta}\right)^{\prime}(t)=\phi^{\prime}\left(t-\eta_{r}\right)$. By Theorem 4.9, we have

$$
L\left(\phi^{\eta}(t), \phi^{\prime}\left(t-\eta_{r}\right)\right) \leq \ell\left(\phi^{\eta}(t), \mu^{*}\left(t-\eta_{r}\right)\right) .
$$

By the Lipschitz continuity of the $\beta_{j}$, we have

$$
\left|\beta_{j}\left(\phi^{\eta}(t)\right)-\beta_{j}\left(\phi\left(t-\eta_{r}\right)\right)\right| \leq \delta_{K}(\eta)
$$

where $\delta_{K}(\eta)$ is independent of $\phi$ and $\delta_{K}(\eta) \rightarrow 0$ as $\eta \rightarrow 0$. We deduce from (44) and (45)

$$
L\left(\phi^{\eta}(t), \phi^{\prime}\left(t-\eta_{r}\right)\right)-L\left(\phi\left(t-\eta_{r}\right), \phi^{\prime}\left(t-\eta_{r}\right)\right) \leq k \delta_{K}(\eta)+\sum_{j} \mu_{j}^{*}\left(t-\eta_{r}\right) \log \frac{\beta_{j}\left(\phi\left(t-\eta_{r}\right)\right)}{\beta_{j}\left(\phi^{\eta}(t)\right)} .
$$

Let

$$
\tilde{v}_{i_{r}}=\left(\frac{3}{\lambda_{1}}\right)^{r-1} v_{i_{r}}+\sum_{j=1}^{r-1}\left(\frac{3}{\lambda_{1}}\right)^{j-1} v_{i_{j}}, \quad \text { and } \hat{v}_{i_{r}}=\frac{\tilde{v}_{i_{r}}}{\left|\tilde{v}_{i_{r}}\right|} \in \mathcal{C}_{1, i_{r}} .
$$

By Assumption 2.3 (B4), there exists a constant $\lambda_{4}>0$ such that for $z \in B_{i_{r}}$ and $\eta<\eta_{2} \leq \eta_{1}$ small enough (note that $\eta_{2}$ depends on $\lambda_{1}$ and $\lambda_{2}$ but not directly on $\phi$, except through $K$ ),

$$
\beta_{j}(z)<\lambda_{4} \Rightarrow \beta_{j}\left(z+\eta \tilde{v}_{i_{r}}\right) \geq \beta_{j}(z)
$$

hence

$$
\log \frac{\beta_{j}\left(\phi\left(t-\eta_{r}\right)\right)}{\beta_{j}\left(\phi^{\eta}(t)\right)}<0 \quad \text { if } \beta_{j}\left(\phi\left(t-\eta_{r}\right)\right)<\lambda_{4} .
$$

If $\beta_{j}\left(\phi\left(t-\eta_{r}\right)\right) \geq \lambda_{4}$ (recall the definition of $\delta_{K}(\eta)$ and choose $\eta<\eta_{3}<\eta_{2}$ small enough such that $\left.\delta_{K}(\eta)<\lambda_{4} / 2\right)$

$$
\begin{aligned}
\log \frac{\beta_{j}\left(\phi\left(t-\eta_{r}\right)\right)}{\beta_{j}\left(\phi^{\eta}(t)\right)} & \leq \log \frac{\beta_{j}\left(\phi\left(t-\eta_{r}\right)\right)}{\beta_{j}\left(\phi\left(t-\eta_{r}\right)\right)-\delta_{K}(\eta)} \\
& \leq \log \frac{\lambda_{4}}{\lambda_{4}-\delta_{K}(\eta)}
\end{aligned}
$$




$$
\begin{aligned}
& =\log \frac{1}{1-\delta_{K}(\eta) / \lambda_{4}} \\
& \leq \frac{2 \delta_{K}(\eta)}{\lambda_{4}},
\end{aligned}
$$

since $\log (1 /(1-x))<2 x$ for $0<x \leq 1 / 2$.

From Lemma 4.3 and Lemma 4.5, there exist (universal, i.e., independent of $x$ ) constants $B \geq B_{1} \vee B_{2}, B>1, C_{1}, C_{3}$ such that for $|y| \geq B$, and $x \in A$,

$$
\begin{gathered}
L(x, y) \geq C_{1}|y| \log |y|, \\
\left|\mu^{*}\right|=\left|\mu^{*}(x, y)\right| \leq C_{3}|y| .
\end{gathered}
$$

Hence if $\left|\phi^{\prime}\left(t-\eta_{r}\right)\right| \geq B$, using (46), (47), (48) and (50) for the first inequality and (49) for the second, we get

$$
\begin{aligned}
L\left(\phi^{\eta}(t), \phi^{\prime}\left(t-\eta_{r}\right)\right)-L\left(\phi\left(t-\eta_{r}\right), \phi^{\prime}\left(t-\eta_{r}\right)\right) & \leq k \delta_{K}(\eta)+k C_{3}\left|\phi^{\prime}\left(t-\eta_{r}\right)\right| \frac{2 \delta_{K}(\eta)}{\lambda_{4}} \\
& \leq k \delta_{K}(\eta)+k C_{3} \frac{2 \delta_{K}(\eta) L\left(\phi\left(t-\eta_{r}\right), \phi^{\prime}\left(t-\eta_{r}\right)\right)}{C_{1} \lambda_{4} \log \left|\phi^{\prime}\left(t-\eta_{r}\right)\right|} .
\end{aligned}
$$

If however $\left|\phi^{\prime}\left(t-\eta_{r}\right)\right|<B$, Lemma 4.5 implies similarly as before that $\left|\mu^{*}\left(t-\eta_{r}\right)\right| \leq C_{3} B$. From (46), we deduce

$$
L\left(\phi^{\eta}(t), \phi^{\prime}\left(t-\eta_{r}\right)\right)-L\left(\phi\left(t-\eta_{r}\right), \phi^{\prime}\left(t-\eta_{r}\right)\right) \leq k \delta_{K}(\eta)+k C_{3} B \frac{2 \delta_{K}(\eta)}{\lambda_{4}} .
$$

Inequalities (51) and (52) imply

$$
L\left(\phi^{\eta}(t), \phi^{\prime}\left(t-\eta_{r}\right)\right)-L\left(\phi\left(t-\eta_{r}\right), \phi^{\prime}\left(t-\eta_{r}\right)\right) \leq \delta_{1, K}(\eta)+\delta_{2, K}(\eta) L\left(\phi\left(t-\eta_{r}\right), \phi^{\prime}\left(t-\eta_{r}\right)\right)
$$

with constants $\delta_{i, K}(\eta) \rightarrow 0$ as $\eta \rightarrow 0$. We can hence choose $\eta<\eta_{4}<\eta_{3}$ small enough such that

$$
\sum_{r=1}^{J} \int_{t_{r-1}+\eta_{r}}^{t_{r}+\eta_{r}} L\left(\phi^{\eta}(t), \phi^{\prime}\left(t-\eta_{r}\right)\right) d t-\sum_{r=1}^{J} \int_{t_{r-1}+\eta_{r}}^{t_{r}+\eta_{r}} L\left(\phi\left(t-\eta_{r}\right), \phi^{\prime}\left(t-\eta_{r}\right)\right) d t<\frac{\epsilon}{2} .
$$

This yields the result.

The following lemma is the main difference to the corresponding result of Shwartz and Weiss [2005]. We transform the LLN from Assumption 2.3 (C) to a LDP lower bound for linear functions following the vector $v_{i}$ near the boundary.

In the next statement, $\alpha$ is the exponent which appears in the Assumption 2.3 (C).

Lemma 5.7. Assume that Assumption 2.3 holds. Let $i \leq I_{1}, x \in A \cap B_{i}$ and $x^{N} \in A^{N} \cap B_{i}$ such that

$$
\limsup _{N \rightarrow \infty}\left|x^{N}-x\right| N^{\alpha}<1 .
$$

Let furthermore $\epsilon>0$ and define $\mu^{i}, \phi^{x}$ and $\eta_{0}$ as in Assumption 2.3 (C). Then for all $\eta$ small enough, all $\epsilon$,

$$
\liminf _{N \rightarrow \infty} \frac{1}{N} \log \mathbb{P}\left[\sup _{t \in[0, \eta]}\left|Z^{N, x^{N}}(t)-\phi^{x}(t)\right|<\epsilon\right] \geq-\int_{0}^{\eta} \ell\left(\phi^{x}(t), \mu^{i}\right) d t,
$$

and the above convergence is uniform in $x \in A$. 
Proof. The proof follows the same line of reasoning as the proof of Proposition 5.1 but is technically more involved.

For simplicity, let $N$ be large enough and $\eta<\eta_{0}$ (for $\eta_{0}$ as in Assumption 2.3 (C)) be small enough such that $\phi^{x^{N}}(t) \in B_{i}$ for all $t \leq \eta$. We furthermore let

$$
\tilde{\epsilon}<\epsilon_{1}:=\epsilon \wedge \lambda_{1} \eta
$$

Define the set

$$
F^{N}:=\left\{\sup _{t \in[0, \eta]}\left|Z^{N, x^{N}}(t)-\phi^{x}(t)\right|<\tilde{\epsilon}\right\} .
$$

Let $\xi_{\eta}=\xi_{\eta}^{N}=\left.\frac{d \tilde{\mathbb{P}}}{d \mathbb{P}}\right|_{\mathcal{F}_{\eta}}$ be given as in Theorem A.1 for the rates $\tilde{\beta}_{j}=\tilde{\mu}_{j}^{i}$. We note that due to Assumption $2.3(\mathrm{C})$,

$$
\tilde{\mathbb{P}}\left[F^{N}\right] \geq 1-\delta(N, \tilde{\epsilon}) \rightarrow 1 \quad \text { as } N \rightarrow \infty .
$$

From Corollary A.2, (53) and Jensen's inequality, we deduce that

$$
\begin{aligned}
& \liminf _{N \rightarrow \infty} \frac{1}{N} \log \mathbb{P}\left[\sup _{t \in[0, \eta]}\left|Z^{N, x^{N}}(t)-\phi^{x}(t)\right|<\epsilon\right] \\
& \quad \geq \liminf _{N \rightarrow \infty} \frac{1}{N} \log \mathbb{P}\left[F^{N}\right] \\
& \quad \geq \liminf _{N \rightarrow \infty} \frac{1}{N} \log \tilde{\mathbb{E}}\left[\xi_{\eta}^{-1} \mathbb{1}_{F^{N}}\right] \\
& \quad=\liminf _{N \rightarrow \infty} \frac{1}{N} \log \left\{\tilde{\mathbb{P}}\left[F^{N}\right] \tilde{\mathbb{E}}_{F^{N}}\left[\exp \left(X_{\eta}\right)\right]\right\} \\
& \quad \geq \liminf _{N \rightarrow \infty} \frac{1}{N} \log \tilde{\mathbb{P}}\left[F^{N}\right]+\liminf _{N \rightarrow \infty} \frac{1}{N} \log \tilde{\mathbb{E}}_{F^{N}}\left[\exp \left(X_{\eta}\right)\right] \\
& \geq \liminf _{N \rightarrow \infty} \tilde{\mathbb{E}}_{F^{N}}\left[\frac{X_{\eta}}{N}\right]
\end{aligned}
$$

where $\tilde{\mathbb{E}}_{F^{N}}[X]:=\tilde{\mathbb{E}}\left[X \mid F^{N}\right]$ and

$$
\begin{aligned}
X_{\eta}^{N}:=X_{\eta}:=\log \xi_{\eta}^{-1}=\sum_{\tau \leq \eta} & {\left[\operatorname { l o g } \beta _ { j ( \tau ) } \left(Z^{N, x^{N}}(\tau-)-\log \tilde{\mu}_{j(\tau)}^{i}\left(Z^{N, x^{N}}(\tau-)\right]\right.\right.} \\
+ & N \sum_{j} \int_{0}^{\eta}\left(\tilde{\mu}_{j}^{i}\left(Z^{N, x^{N}}(t)-\beta_{j}\left(Z^{N, x^{N}}(t)\right)\right) d t .\right.
\end{aligned}
$$

We have $\operatorname{dist}\left(\phi^{x^{N}}(t), \partial A\right) \geq \lambda_{1} t$ (cf. Assumption $\left.2.3(\mathrm{~A} 3)\right)$ and therefore on $F^{N}$,

$$
\operatorname{dist}\left(Z^{N, x^{N}}(t), \partial A\right)>\lambda_{1} t-\tilde{\epsilon} \quad \text { for } t \in\left[\frac{\tilde{\epsilon}}{\lambda_{1}}, \eta\right] .
$$

Consequently

$$
\tilde{\mu}_{j}^{i}\left(Z^{N, x^{N}}(t)\right)=\mu_{j}^{i} \quad \text { for all } j \text { and for all } t \in\left[\frac{\tilde{\epsilon}}{\lambda_{1}}, \eta\right]
$$

We obtain

$$
\begin{aligned}
& \frac{1}{\tilde{\mathbb{P}}\left[F^{N}\right]} \tilde{\mathbb{E}}\left[\mathbb{1}_{F^{N}} \int_{0}^{\eta} \sum_{j} \tilde{\mu}_{j}^{i}\left(Z^{N, x^{N}}(t)\right) d t\right] \\
& =\frac{1}{\tilde{\mathbb{P}}\left[F^{N}\right]}\left(\tilde{\mathbb{E}}\left[\mathbb{1}_{F^{N}} \int_{\tilde{\epsilon} / \lambda_{1}}^{\eta} \sum_{j=1}^{k} \tilde{\mu}_{j}^{i}\left(Z^{N, x^{N}}(t)\right) d t\right]+\tilde{\mathbb{E}}\left[\mathbb{1}_{F^{N}} \int_{0}^{\tilde{\epsilon} / \lambda_{1}} \sum_{j=1}^{k} \tilde{\mu}_{j}^{i}\left(Z^{N, x^{N}}(t)\right) d t\right]\right)
\end{aligned}
$$




$$
=: \sum_{j=1}^{k} \int_{\tilde{\epsilon} / \lambda_{1}}^{\eta} \mu_{j}^{i} d t+X_{1}^{N}(\tilde{\epsilon})
$$

since $\mu_{j}^{i}\left(Z^{N, x^{N}}(t)\right)=\mu_{j}^{i}$ on $F^{N}$. We note that for all $N$,

$$
\left|X_{1}^{N}(\tilde{\epsilon})\right| \leq \frac{\tilde{\epsilon}}{\lambda_{1}} k \bar{\mu} \quad \text { where } \bar{\mu}:=\max _{j=1, \ldots, k} \mu_{j}^{i}
$$

By the fact that the $\beta_{j}$ 's are bounded and continuous and by Theorem 3.1, we have for $j=1, \ldots, k$,

$$
\sup _{t \in[0, \eta]}\left|\beta_{j}\left(Z^{N, x^{N}}(t)\right)-\beta_{j}\left(\phi^{x}(t)\right)\right| \rightarrow 0 \quad \text { a.s. as } N \rightarrow \infty .
$$

Combined with (53), this implies

$$
\frac{1}{\tilde{\mathbb{P}}\left[F^{N}\right]} \tilde{\mathbb{E}}\left[\mathbb{1}_{F^{N}} \int_{0}^{\eta} \sum_{j=1}^{k} \beta_{j}\left(Z^{N, x^{N}}(t)\right) d t\right] \longrightarrow \sum_{j=1}^{k} \int_{0}^{\eta} \beta_{j}\left(\phi^{x}(t)\right) d t
$$

as $N \rightarrow \infty$.

Let us now define the following processes. For $z \in A, j=1, \ldots, k$ and $0 \leq s<t \leq \eta$ let $\bar{Z}^{N, z}$ solves Equation (3) with constant rates $\mu_{j}^{i}$ under $\tilde{\mathbb{P}}$, and

$$
\begin{aligned}
Y_{j}^{N, z, s, t} & :=\frac{1}{N} \cdot \# \text { jumps of } Z^{N, z} \text { in direction } h_{j} \text { in }[s, t], \\
\bar{Y}_{j}^{N, z, s, t} & :=\frac{1}{N} \cdot \text { \#jumps of } \bar{Z}^{N, z} \text { in direction } h_{j} \text { in }[s, t],
\end{aligned}
$$

We have for any event $F$, noting that $\tilde{\mathbb{P}}[F]-\tilde{\mathbb{P}}[F]^{2}=\tilde{\mathbb{P}}\left[F^{c}\right]-\tilde{\mathbb{P}}\left[F^{c}\right]^{2}$,

$$
\begin{aligned}
\tilde{\mathbb{E}}\left[Y_{j}^{N, z, s, t}\right] & \leq(t-s) \mu_{j}^{i}=\tilde{\mathbb{E}}\left[\bar{Y}_{j}^{N, z, s, t}\right], \\
\widetilde{\operatorname{Var}}\left[Y_{j}^{N, z, s, t}\right] & \leq(t-s) \mu_{j}^{i}=\widetilde{\operatorname{Var}}\left[\bar{Y}_{j}^{N, z, s, t}\right], \\
\widetilde{\operatorname{Cov}}\left(\mathbb{1}_{F}, Y_{j}^{N, z, s, t}\right)|,| \widetilde{\operatorname{Cov}}\left(\mathbb{1}_{F}, \bar{Y}_{j}^{N, z, s, t}\right) \mid & \leq \sqrt{\widetilde{\operatorname{Var}}\left[\mathbb{1}_{F}\right]} \sqrt{\widetilde{\operatorname{Var}}\left[\bar{Y}_{j}^{N, z, s, t}\right]} \\
& \leq \sqrt{\tilde{\mathbb{P}}[F]-\tilde{\mathbb{P}}[F]^{2}} \sqrt{(t-s) \mu_{j}^{i}} .
\end{aligned}
$$

We define the sets

$$
F_{1}^{N}:=\left\{\left|Z^{N, x^{N}}\left(\frac{2 \tilde{\epsilon}}{\lambda_{1}}\right)-\phi^{x}\left(\frac{2 \tilde{\epsilon}}{\lambda_{1}}\right)\right|<\frac{\tilde{\epsilon}}{2}\right\} \in \mathcal{F}_{2 \tilde{\epsilon} / \lambda_{1}}
$$

and for $z \in A$ with $\left|z-\phi^{x}\left(2 \tilde{\epsilon} / \lambda_{1}\right)\right|<\tilde{\epsilon} / 2$,

$$
F_{2}^{N, z}:=\left\{\sup _{t \in\left[0, \eta-2 \tilde{\epsilon} / \lambda_{1}\right]}\left|Z^{N, z}(t)-\phi^{z}(t)\right|<\frac{\tilde{\epsilon}}{2}\right\} .
$$

Note that

$$
\operatorname{dist}\left(\phi^{x}(t), \partial A\right) \geq 2 \tilde{\epsilon} \quad \text { for } t \in\left[\frac{2 \tilde{\epsilon}}{\lambda_{1}}, \eta\right]
$$


and whenever $\left|z-\phi^{x}\left(2 \tilde{\epsilon} / \lambda_{1}\right)\right|<\tilde{\epsilon} / 2$,

$$
\left|\phi^{z}(t)-\phi^{x}\left(t+\frac{2 \tilde{\epsilon}}{\lambda_{1}}\right)\right|<\frac{\tilde{\epsilon}}{2} \quad \text { for } t \in\left[0, \eta-\frac{2 \tilde{\epsilon}}{\lambda_{1}}\right] .
$$

Hence

$$
\operatorname{dist}\left(Z^{N, z}(t), \partial A\right) \geq \tilde{\epsilon} \quad \text { for } t \in\left[0, \eta-\frac{2 \tilde{\epsilon}}{\lambda_{1}}\right]
$$

and therefore $Z^{N, z}(t)=\bar{Z}^{N, z}(t)$ on $F_{2}^{N, z}$. This implies

$$
F_{2}^{N, z}=\left\{\sup _{t \in\left[0, \eta-2 \tilde{\epsilon} / \lambda_{1}\right]}\left|\bar{Z}^{N, z}(t)-\phi^{z}(t)\right|<\frac{\tilde{\epsilon}}{2}\right\} .
$$

We now let

$$
\frac{2 \tilde{\epsilon}}{\lambda_{1}} \leq s<t \leq \eta
$$

and compute (by using the Markov property of $Z^{N}$ and the fact that $Y_{j}^{N, z, s-2 \tilde{\epsilon} / \lambda_{1}, t-2 \tilde{\epsilon} / \lambda_{1}}=$ $\bar{Y}_{j}^{N, z, s-2 \tilde{\epsilon} / \lambda_{1}, t-2 \tilde{\epsilon} / \lambda_{1}}$ on the event $\left.F_{2}^{N, z}\right)$

$$
\begin{aligned}
& \tilde{\mathbb{E}}\left[Y_{j}^{N, x^{N}, s, t}\right]=\tilde{\mathbb{E}}\left[\mathbb{1}_{F_{1}^{N}} Y_{j}^{N, x^{N}, s, t}\right]+\tilde{\mathbb{E}}\left[\mathbb{1}_{\left(F_{1}^{N}\right)^{c}} Y_{j}^{N, x^{N}, s, t}\right] \\
& \geq \tilde{\mathbb{P}}\left[F_{1}^{N}\right] \cdot \inf _{z ;\left|z-\phi^{x}\left(\tilde{\epsilon} / \lambda_{1}\right)\right|<\tilde{\epsilon} / 2} \tilde{\mathbb{E}}\left[Y_{j}^{N, z, s-2 \tilde{\epsilon} / \lambda_{1}, t-2 \tilde{\epsilon} / \lambda_{1}}\right]+\tilde{\mathbb{E}}\left[\mathbb{1}_{\left(F_{1}^{N}\right)^{c}} Y_{j}^{N, x^{N}, s, t}\right] \\
& \geq \tilde{\mathbb{P}}\left[F_{1}^{N}\right] \cdot \inf _{z ;\left|z-\phi^{x}\left(\tilde{\epsilon} / \lambda_{1}\right)\right|<\tilde{\epsilon} / 2} \tilde{\mathbb{E}}\left[\mathbb{1}_{F_{2}^{N, z}} Y_{j}^{N, z, s-2 \tilde{\epsilon} / \lambda_{1}, t-2 \tilde{\epsilon} / \lambda_{1}}\right] \\
& +\tilde{\mathbb{P}}\left[F_{1}^{N}\right] \cdot \inf _{z ;\left|z-\phi^{x}\left(\tilde{\epsilon} / \lambda_{1}\right)\right|<\tilde{\epsilon} / 2} \tilde{\mathbb{E}}\left[\mathbb{1}_{\left(F_{2}^{N, z}\right)^{c}} Y_{j}^{N, z, s-2 \tilde{\epsilon} / \lambda_{1}, t-2 \tilde{\epsilon} / \lambda_{1}}\right] \\
& +\tilde{\mathbb{E}}\left[\mathbb{1}_{\left(F_{1}^{N}\right)^{c}} Y_{j}^{N, x^{N}, s, t}\right] \\
& =\mu_{j}^{i}(t-s) \tilde{\mathbb{P}}\left[F_{1}^{N}\right] \cdot \inf _{z ;\left|z-\phi^{x}\left(\tilde{\epsilon} / \lambda_{1}\right)\right|<\tilde{\epsilon} / 2} \tilde{\mathbb{P}}\left[F_{2}^{N, z}\right] \\
& +\tilde{\mathbb{P}}\left[F_{1}^{N}\right] \cdot \inf _{z ;\left|z-\phi^{x}\left(\tilde{\epsilon} / \lambda_{1}\right)\right|<\tilde{\epsilon} / 2} \widetilde{\operatorname{Cov}}\left(\mathbb{1}_{F_{2}^{N, z}}, Y_{j}^{N, z, s-2 \tilde{\epsilon} / \lambda_{1}, t-2 \tilde{\epsilon} / \lambda_{1}}\right) \\
& +\tilde{\mathbb{P}}\left[F_{1}^{N}\right] \cdot \inf _{z ;\left|z-\phi^{x}\left(\tilde{\epsilon} / \lambda_{1}\right)\right|<\tilde{\epsilon} / 2} \tilde{\mathbb{E}}\left[\mathbb{1}_{\left(F_{2}^{N, z}\right)^{c}} Y_{j}^{N, z, s-2 \tilde{\epsilon} / \lambda_{1}, t-2 \tilde{\epsilon} / \lambda_{1}}\right] \\
& +\tilde{\mathbb{E}}\left[\mathbb{1}_{\left(F_{1}^{N}\right)^{c}} Y_{j}^{N, x^{N}, s, t}\right] \\
& \geq \mu_{j}^{i}(t-s) \tilde{\mathbb{P}}\left[F_{1}^{N}\right] \cdot \inf _{z ;\left|z-\phi^{x}\left(\tilde{\epsilon} / \lambda_{1}\right)\right|<\tilde{\epsilon} / 2} \tilde{\mathbb{P}}\left[F_{2}^{N, z}\right] \\
& -\tilde{\mathbb{P}}\left[F_{1}^{N}\right] \cdot \inf _{z ;\left|z-\phi^{x}\left(\tilde{\epsilon} / \lambda_{1}\right)\right|<\tilde{\epsilon} / 2}\left|\widetilde{\operatorname{Cov}}\left(\mathbb{1}_{F_{2}^{N, z}}, Y_{j}^{N, z, s-2 \tilde{\epsilon} / \lambda_{1}, t-2 \tilde{\epsilon} / \lambda_{1}}\right)\right|
\end{aligned}
$$

as the third and the fourth term in (62) are non-negative. As furthermore

$$
\tilde{\mathbb{P}}\left[F_{1}^{N}\right] \cdot \inf _{z ;\left|z-\phi^{x}\left(\tilde{\epsilon} / \lambda_{1}\right)\right|<\tilde{\epsilon} / 2} \tilde{\mathbb{P}}\left[F_{2}^{N, z}\right] \rightarrow 1
$$

and

$$
\tilde{\mathbb{P}}\left[F_{1}^{N}\right] \cdot \inf _{z ;\left|z-\phi^{x}\left(\tilde{\epsilon} / \lambda_{1}\right)\right|<\tilde{\epsilon} / 2} \widetilde{\operatorname{Cov}}\left(\mathbb{1}_{F_{2}^{N, z}}, Y_{j}^{N, z, s-2 \tilde{\epsilon} / \lambda_{1}, t-2 \tilde{\epsilon} / \lambda_{1}}\right) \rightarrow 0
$$

as $N \rightarrow \infty$ by Assumption 2.3 (C), Theorem 3.1 and (60) . Combinig the resulting inequality with (58) for all $\tilde{\epsilon}<\epsilon_{1}$ and $2 \tilde{\epsilon} / \lambda_{1} \leq s<t$, we deduce that

$$
\lim _{N \rightarrow \infty} \tilde{\mathbb{E}}\left[Y_{j}^{N, x^{N}, s, t}\right]=\mu_{j}^{i}(t-s) .
$$


Note that for $0 \leq s<t \leq \eta$, and all $\tilde{\epsilon}<\epsilon_{1}$

$$
Y_{j}^{N, x^{N}, s, t}=Y_{j}^{N, x^{N}, s,\left(s \vee 2 \tilde{\epsilon} / \lambda_{1}\right) \wedge t}+Y_{j}^{N, x^{N},\left(s \vee 2 \tilde{\epsilon} / \lambda_{1}\right) \wedge t, t}
$$

and hence also for $0 \leq s<t \leq \eta$, since when $s<2 \tilde{\epsilon} / \lambda_{1}, Y_{j}^{N, x^{N}, s, 2 \tilde{\epsilon} / \lambda_{1} \wedge t}$ is of the order of $\tilde{\epsilon}$,

$$
\lim _{N \rightarrow \infty} \tilde{\mathbb{E}}\left[Y_{j}^{N, x^{N}, s, t}\right]=\mu_{j}^{i}(t-s)
$$

Let now $\tau_{j} \in[0, \eta]$ denote the jump times of $Z^{N, x^{N}}$ in direction $h_{j}$. Since $\tilde{\mu}_{j}^{i}\left(Z^{N, x^{N}}\left(\tau_{j}-\right)\right)=$ $\log \mu_{j}^{i} \tilde{\mathbb{P}}$ a.s.,

$$
\begin{aligned}
\frac{1}{\tilde{\mathbb{P}}\left[F^{N}\right]} \sum_{j ; \mu_{j}^{i}>0} \tilde{\mathbb{E}}\left[\frac{1}{N} \mathbb{1}_{F^{N}} \sum_{\tau_{j} \leq \eta} \log \tilde{\mu}_{j}^{i}\left(Z^{N, x^{N}}\left(\tau_{j}-\right)\right)\right] \\
=\frac{1}{\tilde{\mathbb{P}}\left[F^{N}\right]} \sum_{j ; \mu_{j}^{i}>0} \log \mu_{j}^{i} \tilde{\mathbb{E}}\left[\mathbb{1}_{F^{N}} Y_{j}^{N, x^{N}, 0, \eta}\right] \\
=\frac{1}{\tilde{\mathbb{P}}\left[F^{N}\right]} \sum_{j ; \mu_{j}^{i}>0} \log \mu_{j}^{i}\left\{\tilde{\mathbb{P}}\left[F^{N}\right] \cdot \tilde{\mathbb{E}}\left[Y_{j}^{N, x^{N}, 0, \eta}\right]+\widetilde{\operatorname{Cov}}\left(\mathbb{1}_{F^{N}}, Y_{j}^{N, x^{N}, 0, \eta}\right)\right\} \\
\longrightarrow \sum_{j ; \mu_{j}^{i}>0} \eta \mu_{j}^{i} \log \mu_{j}^{i}=\sum_{j=1}^{k} \int_{0}^{\eta} \mu_{j}^{i} \log \mu_{j}^{i} d t
\end{aligned}
$$

as $N \rightarrow \infty$ by (53), (60) and (64).

For the last and most extensive step of the proof, we define for $\tilde{\epsilon}<\epsilon_{1}$ and (cf. Assumption 2.3 (C) and (11))

$$
\epsilon_{N}=\frac{1}{N^{\alpha}}
$$

and the set

$$
\tilde{F}^{N}:=\left\{\sup _{t \in[0, \eta]}\left|Z^{N, x^{N}}(t)-\phi^{x}(t)\right|<\epsilon_{N}\right\}
$$

We assume w.l.o.g. that from now on $N$ is large enough (cf. Assumption 2.3 (C)) such that

$$
\tilde{\mathbb{P}}\left[\tilde{F}^{N}\right] \geq \tilde{\mathbb{P}}\left[\sup _{t \in[0, \eta]}\left|Z^{N, x^{N}}(t)-\phi^{x^{N}}(t)\right|<\epsilon_{N}\right] \geq 1-\delta\left(N, \epsilon_{N}\right)
$$

where $\delta\left(N, \epsilon_{N}\right) \rightarrow 0$ as $N \rightarrow \infty$. We note that we have for all $\tilde{\epsilon} \leq \epsilon_{1}, j=1, \ldots, k$ with $\mu_{j}^{i}>0, N \in \mathbb{N}$ and $t \in\left[2 \tilde{\epsilon} / \lambda_{1}, \eta\right]$,

$$
\log \beta_{j}\left(\phi^{x}(t)\right), \log \beta_{j}\left(Z^{N, x^{N}}(t)\right) \geq \log \underline{\beta}(\tilde{\epsilon})>0 \quad \text { on } F^{N} \text { and } \tilde{F}^{N} .
$$

We compute

$$
\begin{aligned}
\frac{1}{\tilde{\mathbb{P}}\left[F^{N}\right]} \tilde{\mathbb{E}}\left[\frac{1}{N} \mathbb{1}_{F^{N}} \sum_{\tau \leq \eta} \log \beta_{j(\tau)}\left(Z^{N, x^{N}}(\tau-)\right)\right] \\
=\frac{1}{\tilde{\mathbb{P}}\left[F^{N}\right]} \sum_{j, \mu_{j}^{i}>0} \tilde{\mathbb{E}}\left[\frac{1}{N} \mathbb{1}_{\tilde{F}^{N}} \sum_{\tau_{j} \in\left[2 \tilde{\epsilon} / \lambda_{1}, \eta\right]} \log \beta_{j}\left(Z^{N, x^{N}}\left(\tau_{j}-\right)\right)\right]
\end{aligned}
$$




$$
\begin{aligned}
& +\frac{1}{\tilde{\mathbb{P}}\left[F^{N}\right]} \sum_{j, \mu_{j}^{i}>0} \tilde{\mathbb{E}}\left[\frac{1}{N} \mathbb{1}_{\tilde{F}^{N}} \sum_{\tau_{j} \in\left[0,2 \tilde{\epsilon} / \lambda_{1}\right]} \log \beta_{j}\left(Z^{N, x^{N}}\left(\tau_{j}-\right)\right)\right] \\
& +\frac{1}{\tilde{\mathbb{P}}\left[F^{N}\right]} \sum_{j, \mu_{j}^{i}>0} \tilde{\mathbb{E}}\left[\frac{1}{N} \mathbb{1}_{F^{N} \backslash \tilde{F}^{N}} \sum_{\tau_{j} \in[0, \eta]} \log \beta_{j}\left(Z^{N, x^{N}}\left(\tau_{j}-\right)\right)\right] .
\end{aligned}
$$

Let us first consider the first term in Equation (66). As for all $j$,

$$
\log \beta_{j}(\cdot): \tilde{A}(\tilde{\epsilon}):=\{z \in A \mid \operatorname{dist}(z, \partial A) \geq \tilde{\epsilon}\} \rightarrow \mathbb{R}
$$

is uniformly continuous, there exit constants $\tilde{\delta}_{N}>0$ with $\tilde{\delta}_{N} \downarrow 0$ such that

$$
z, \tilde{z} \in \tilde{A}(\tilde{\epsilon}),|\tilde{z}-z|<3 \epsilon_{N} \Rightarrow\left|\log \beta_{j}(\tilde{z})-\log \beta_{j}(z)\right|<\tilde{\delta}_{N}
$$

We define

$$
M=M(N):=\left\lfloor\left(\eta-2 \tilde{\epsilon} / \lambda_{1}\right) \epsilon_{N}^{-1}+1\right\rfloor
$$

and divide the interval $\left[2 \tilde{\epsilon} / \lambda_{1}, \eta\right]$ into $M$ equidistant subintervals $\left[t_{r}, t_{r+1}\right](r=0, \ldots, M-$ $\left.1, t_{r}=t_{r}(N)\right)$ of length $\Delta=\Delta(N)$, i.e. (for $N$ large enough),

$$
\frac{\epsilon_{N}}{2} \leq \Delta<\epsilon_{N}
$$

For $j=1, \ldots, k, r=0, \ldots, M-1$ and $\tau_{j}, t \in\left[t_{r}, t_{r+1}\right]$ we have,

$$
\left|Z^{N, x^{N}}\left(\tau_{j}-\right)-\phi^{x}(t)\right| \leq 2 \epsilon_{N} \quad \text { on } \tilde{F}^{N}
$$

since $\left|\phi^{x}\left(\tau_{j}\right)-\phi^{x}(t)\right| \leq\left|\tau_{j}-t\right|$ as $\left|v_{i}\right| \leq 1$, and hence (cf. (67))

$$
\inf _{t \in\left[t_{r}, t_{r+1}\right]} \log \beta_{j}\left(\phi^{x}(t)\right)-\tilde{\delta}_{N} \leq \log \beta_{j}\left(Z^{N, x^{N}}\left(\tau_{j}-\right)\right) .
$$

From this inequality, we deduce

$$
\begin{aligned}
\frac{1}{\tilde{\mathbb{P}}\left[F^{N}\right]} \tilde{\mathbb{E}}\left[\frac{1}{N} \mathbb{1}_{\tilde{F}^{N}} \sum_{\tau \in\left[2 \tilde{\epsilon} / \lambda_{1}, \eta\right]} \log \beta_{j(\tau)}\left(Z^{N, x^{N}}(\tau-)\right)\right] \\
=\frac{1}{\tilde{\mathbb{P}}\left[F^{N}\right]} \sum_{j, \mu_{j}^{i}>0} \sum_{r=0}^{M-1} \tilde{\mathbb{E}}\left[\frac{1}{N} \mathbb{1}_{\tilde{F}^{N}} \sum_{\tau_{j} \in\left[t_{r}, t_{r+1}\right)} \log \beta_{j}\left(Z^{N, x^{N}}\left(\tau_{j}-\right)\right)\right] \\
\geq \frac{1}{\tilde{\mathbb{P}}\left[F^{N}\right]} \sum_{j, \mu_{j}^{i}>0} \sum_{r=0}^{M-1}\left(\inf _{t \in\left[t_{r}, t_{r+1}\right)} \log \beta_{j}\left(\phi^{x}(t)\right)-\tilde{\delta}_{N}\right) \tilde{\mathbb{E}}\left[\mathbb{1}_{\tilde{F}^{N}} Y_{j}^{\left.N, x^{N}, t_{r}, t_{r+1}\right]}\right] \\
\geq \frac{1}{\tilde{\mathbb{P}}\left[F^{N}\right]} \sum_{j, \mu_{j}^{i}>0} \sum_{r=0}^{M-1} \inf _{t \in\left[t_{r}, t_{r+1}\right)} \log \beta_{j}\left(\phi^{x}(t)\right) \tilde{\mathbb{P}}\left[\tilde{F}^{N}\right] \tilde{\mathbb{E}}\left[Y_{j}^{\left.N, x^{N}, t_{r}, t_{r+1}\right]}\right. \\
\quad-\frac{1}{\tilde{\mathbb{P}}\left[F^{N}\right]} \sum_{j, \mu_{j}^{i}>0} \sum_{r=0}^{M-1}|\log \underline{\beta}(\tilde{\epsilon})|\left|\widetilde{\operatorname{Cov}}\left(\mathbb{1}_{\tilde{F}^{N}}, Y_{j}^{N, x^{N}, t_{r}, t_{r+1}}\right)\right| \\
\quad-\frac{1}{\tilde{\mathbb{P}}\left[F^{N}\right]} \tilde{\delta}_{N} \sum_{j, \mu_{j}^{i}>0} \tilde{\mathbb{E}}\left[\mathbb{1}_{\tilde{F}^{N}} Y^{N, x^{N}, 2 \tilde{\epsilon} / \lambda_{1}, \eta}\right] .
\end{aligned}
$$


The second term in Inequality (68) satisfies (cf. Inequality (60) and Assumption 2.3 (C); we assume that $N$ is sufficiently large such that $\left.M \leq 2 \epsilon_{N}^{-1} \eta\right)$,

$$
\begin{aligned}
& \frac{1}{\tilde{\mathbb{P}}\left[F^{N}\right]} \sum_{j, \mu_{j}^{i}>0} \sum_{r=0}^{M-1}|\log \underline{\beta}(\tilde{\epsilon})|\left|\widetilde{\operatorname{Cov}}\left(\mathbb{1}_{\tilde{F}^{N}}, Y_{j}^{N, x^{N}, t_{r}, t_{r+1}}\right)\right| \\
& \leq \frac{1}{\tilde{\mathbb{P}}\left[F^{N}\right]} 2 k \eta|\log \underline{\beta}(\tilde{\epsilon})| \epsilon_{N}^{-1} \sqrt{\bar{\mu} \epsilon_{N}} \sqrt{\delta\left(N, \epsilon_{N}\right)} \\
& \quad \rightarrow 0
\end{aligned}
$$

as $N \rightarrow \infty$. The third term in Equation (68) satisfies

$$
\frac{1}{\tilde{\mathbb{P}}\left[F^{N}\right]} \tilde{\delta}_{N} \sum_{j, \mu_{j}^{i}>0} \tilde{\mathbb{E}}\left[\mathbb{1}_{\tilde{F}^{N}} Y^{N, x^{N}, 2 \tilde{\epsilon} / \lambda_{1}, \eta}\right] \leq \frac{1}{\tilde{\mathbb{P}}\left[F^{N}\right]} \tilde{\delta}_{N} k \bar{\mu} \eta \rightarrow 0
$$

as $N \rightarrow \infty$. Finally, let us consider the first term in Equation (68). Recall that by (58) and (63) , we have

$$
\begin{aligned}
& \mu_{j}^{i}\left(t_{r+1}-t_{r}\right) \geq \tilde{\mathbb{E}}\left[Y_{j}^{\left.N, x^{N}, t_{r}, t_{r+1}\right]}\right. \\
& \geq \mu_{j}^{i}\left(t_{r+1}-t_{r}\right) \tilde{\mathbb{P}}\left[F_{1}^{N}\right] \cdot \inf _{z ;\left|z-\phi^{x}\left(\tilde{\epsilon} / \lambda_{1}\right)\right|<\tilde{\epsilon} / 2} \tilde{\mathbb{P}}\left[F_{2}^{N, z}\right] \\
& -\tilde{\mathbb{P}}\left[F_{1}^{N}\right] \cdot \inf _{z ;\left|z-\phi^{x}\left(\tilde{\epsilon} / \lambda_{1}\right)\right|<\tilde{\epsilon} / 2}\left|\widetilde{\operatorname{Cov}}\left(\mathbb{1}_{F_{2}^{N, z}}, Y_{j}^{N, z, t_{r}-2 \tilde{\epsilon} / \lambda_{1}, t_{r+1}-2 \tilde{\epsilon} / \lambda_{1}}\right)\right| .
\end{aligned}
$$

We define

$$
\begin{aligned}
\alpha_{1}^{N}: & :=\frac{\tilde{\mathbb{P}}\left[\tilde{F}^{N}\right]}{\tilde{\mathbb{P}}\left[F^{N}\right]}, \\
\alpha_{2}^{N}: & =\tilde{\mathbb{P}}\left[F_{1}^{N}\right] \cdot \inf _{z ;\left|z-\phi^{x}\left(\tilde{\epsilon} / \lambda_{1}\right)\right|<\tilde{\epsilon} / 2} \tilde{\mathbb{P}}\left[F_{2}^{N, z}\right]<1, \\
\alpha_{3}^{N} & :=\tilde{\mathbb{P}}\left[F_{1}^{N}\right] \cdot \inf _{z ;\left|z-\phi^{x}\left(\tilde{\epsilon} / \lambda_{1}\right)\right|<\tilde{\epsilon} / 2}\left|\widetilde{\operatorname{Cov}}\left(\mathbb{1}_{F_{2}^{N, z}}, Y_{j}^{N, z, t_{r}-2 \tilde{\epsilon} / \lambda_{1}, t_{r+1}-2 \tilde{\epsilon} / \lambda_{1}}\right)\right|, \\
\phi_{j}^{r} & :=\inf _{t \in\left[t_{r}, t_{r+1}\right)} \log \beta_{j}\left(\phi^{x}(t)\right), \\
S^{N} & :=\sum_{j, \mu_{j}^{i}>0} \mu_{j}^{i} \sum_{r=0}^{M-1}\left(t_{r+1}-t_{r}\right) \inf _{t \in\left[t_{r}, t_{r+1}\right)} \log \beta_{j}\left(\phi^{x}(t)\right) .
\end{aligned}
$$

We compute (for $N$ large enough as before)

$$
\begin{aligned}
& \frac{\tilde{\mathbb{P}}\left[\tilde{F}^{N}\right]}{\tilde{\mathbb{P}}\left[F^{N}\right]} \sum_{j, \mu_{j}^{i}>0} \sum_{r=0}^{M-1} \inf _{t \in\left[t_{r}, t_{r+1}\right)} \log \beta_{j}\left(\phi^{x}(t)\right) \tilde{\mathbb{E}}\left[Y_{j}^{\left.N, x^{N}, t_{r}, t_{r+1}\right]}\right. \\
& \geq \alpha_{1}^{N} \sum_{j, \mu_{j}^{i}>0} \sum_{r=0}^{M-1} \inf _{t \in\left[t_{r}, t_{r+1}\right)} \log \beta_{j}\left(\phi^{x}(t)\right) \cdot\left\{\mathbb{1}_{\left\{\phi_{j}^{r}<0\right\}} \mu_{j}\left(t_{r+1}-t_{r}\right)\right. \\
& \left.\quad+\mathbb{1}_{\left\{\phi_{j}^{r}>0\right\}}\left(\alpha_{2}^{N} \mu_{j}\left(t_{r+1}-t_{r}\right)-\alpha_{3}^{N}\right)\right\}
\end{aligned}
$$




$$
\begin{aligned}
& \geq \alpha_{1}^{N} \sum_{j, \mu_{j}^{i}>0} \mu_{j}^{i} \sum_{r=0}^{M-1}\left(t_{r+1}-t_{r}\right) \inf _{t \in\left[t_{r}, t_{r+1}\right)} \log \beta_{j}\left(\phi^{x}(t)\right)\left\{\mathbb{1}_{\left\{\phi_{j}^{r}<0\right\}}+\alpha_{2}^{N} \mathbb{1}_{\left\{\phi_{j}^{r}>0\right\}}\right\} \\
& \quad-2 \eta \alpha_{1}^{N} k|\log \bar{\beta}| \epsilon_{N}^{-1} \alpha_{3}^{N} \\
& \geq \alpha_{1}^{N} S^{N}-2 \eta \alpha_{1}^{N} k|\log \bar{\beta}| \epsilon_{N}^{-1} \alpha_{3}^{N}-\alpha_{1}^{N} k|\log \bar{\beta}| \bar{\mu} \eta\left(1-\alpha_{2}^{N}\right) .
\end{aligned}
$$

We readily observe that

$$
\alpha_{1}^{N}, \alpha_{2}^{N} \rightarrow 1 \quad \text { as } N \rightarrow \infty
$$

by Theorem 3.1 and Assumption 2.3 (C). We furthermore note that by Assumption 2.3 (C) and Theorem 3.1 (cf. also the comment corresponding to (10) and again the fact that the rate of convergence in Theorem 3.1 is independent of initial values),

$$
\frac{\inf _{z} \sqrt{\tilde{\mathbb{P}}\left[F_{2}^{N, z}\right]-\tilde{\mathbb{P}}\left[F_{2}^{N, z}\right]^{2}}}{\sqrt{\epsilon_{N}}} \leq \frac{\inf _{z} \sqrt{\tilde{\mathbb{P}}\left[\left(F_{2}^{N, z}\right) c\right]}}{\sqrt{\epsilon_{N}}} \rightarrow 0 \text { as } N \rightarrow \infty .
$$

Therefore (for $N$ sufficiently large as before),

$$
\begin{aligned}
2 \eta \alpha_{1}^{N} k|\log \bar{\beta}| \epsilon_{N}^{-1} \alpha_{3}^{N} & \leq 2 \eta \alpha_{1}^{N} k|\log \bar{\beta}| \tilde{\mathbb{P}}\left[F_{1}^{N}\right] \sqrt{\bar{\mu}} \frac{1}{\sqrt{\epsilon_{N}}} \inf _{z ;\left|z-\phi^{x}\left(\tilde{\epsilon} / \lambda_{1}\right)\right|<\tilde{\epsilon} / 2} \sqrt{\tilde{\mathbb{P}}\left[F_{2}^{N, z}\right]-\tilde{\mathbb{P}}\left[F_{2}^{N, z}\right]^{2}} \\
& \rightarrow 0
\end{aligned}
$$

as $N \rightarrow 0$. Finally, $S^{N}$ is a Riemann sum and we have

$$
S^{N} \rightarrow \sum_{j} \mu_{j}^{i} \int_{2 \tilde{\epsilon} / \lambda_{1}}^{\eta} \log \beta_{j}\left(\phi^{x}(t)\right) d t \quad \text { as } N \rightarrow \infty .
$$

We observe that (68) - (174) yield

$$
\begin{aligned}
& \liminf _{N \rightarrow \infty} \frac{1}{\tilde{\mathbb{P}}\left[F^{N}\right]} \sum_{j, \mu_{j}^{i}>0} \tilde{\mathbb{E}}\left[\frac{1}{N} \mathbb{1}_{\tilde{F}^{N}} \sum_{\tau_{j} \in\left[2 \tilde{\epsilon} / \lambda_{1}, \eta\right]} \log \beta_{j}\left(Z^{N, x^{N}}\left(\tau_{j}-\right)\right)\right] \\
& \geq \sum_{j=1}^{k} \mu_{j}^{i} \int_{2 \tilde{\epsilon} / \lambda_{1}}^{\eta} \log \beta_{j}\left(\phi^{x}(t)\right) d t .
\end{aligned}
$$

We now consider the second term in the right ahnd side of (66). We define

$$
\tilde{M}=\tilde{M}(N):=\left\lfloor 2 \tilde{\epsilon} \epsilon_{N}^{-1}+1\right\rfloor
$$

and divide the interval $\left[0,2 \tilde{\epsilon} / \lambda_{1}\right]$ into $\tilde{M}$ subintervals $\left[\tilde{t}_{r}, \tilde{t}_{r+1}\right]\left(r=0, \ldots, \tilde{M}-1, \tilde{t}_{r}=\right.$ $\left.\tilde{t}_{r}(N)\right)$ of length $\tilde{\Delta}=\tilde{\Delta}(N)$, i.e., for $N$ large enough,

$$
\frac{\epsilon_{N}}{2 \lambda_{1}} \leq \tilde{\Delta}<\frac{\epsilon_{N}}{\lambda_{1}}
$$

For $r=0, \ldots, \tilde{M}-1$ and $\tau_{j} \in\left[\tilde{t}_{r}, \tilde{t}_{r+1}\right]$, we obtain on $\tilde{F}^{N}$,

$$
\operatorname{dist}\left(Z^{N, x^{N}}\left(\tau_{j}-\right), \partial A\right)>\operatorname{dist}\left(\phi^{x}\left(\tau_{j}-\right), \partial A\right)-2 \epsilon_{N}
$$




$$
\begin{aligned}
& \geq \operatorname{dist}\left(\phi^{x}\left(\tilde{t}_{r}\right), \partial A\right)-2 \epsilon_{N} \\
& \geq \lambda_{1} \tilde{t}_{r}-2 \epsilon_{N} \\
& \geq \frac{r-4}{2} \epsilon_{N} .
\end{aligned}
$$

Hence, $\operatorname{dist}\left(Z^{N, x^{N}}\left(\tau_{j}-\right), \partial A\right)>\epsilon_{N}$ for $r \geq 6$. We compute for $j$ with $\mu_{j}>0$,

$$
\begin{aligned}
\frac{1}{\tilde{\mathbb{P}}\left[F^{N}\right]} \tilde{\mathbb{E}}\left[\frac{1}{N} \mathbb{1}_{\tilde{F}^{N}} \sum_{\tau_{j} \in\left[0,2 \tilde{\epsilon} / \lambda_{1}\right]} \log \beta_{j}\left(Z^{N, x^{N}}\left(\tau_{j}-\right)\right)\right] \\
=\frac{1}{\tilde{\mathbb{P}}\left[F^{N}\right]} \sum_{r=0}^{\tilde{M}-1} \tilde{\mathbb{E}}\left[\frac{1}{N} \mathbb{1}_{\tilde{F}^{N}} \sum_{\tau_{j} \in\left[\tilde{t}_{r}, \tilde{t}_{r+1}\right]} \log \beta_{j}\left(Z^{N, x^{N}}\left(\tau_{j}-\right)\right)\right] \\
=\frac{1}{\tilde{\mathbb{P}}\left[F^{N}\right]} \sum_{r=6}^{\tilde{M}-1} \tilde{\mathbb{E}}\left[\frac{1}{N} \mathbb{1}_{\tilde{F}^{N}} \sum_{\tau_{j} \in\left[\tilde{t}_{r}, \tilde{t}_{r+1}\right]} \log \beta_{j}\left(Z^{N, x^{N}}\left(\tau_{j}-\right)\right)\right] \\
\quad+\frac{1}{\tilde{\mathbb{P}}\left[F^{N}\right]} \sum_{r=0}^{5} \tilde{\mathbb{E}}\left[\frac{1}{N} \mathbb{1}_{\tilde{F}^{N}} \sum_{\tau_{j} \in\left[\tilde{t}_{r}, \tilde{t}_{r+1}\right]} \log \beta_{j}\left(Z^{N, x^{N}}\left(\tau_{j}-\right)\right)\right] .
\end{aligned}
$$

We note that for all $j, \beta_{j}\left(Z^{N, x^{N}}\left(\tau_{j}-\right)\right) \geq \underline{\beta}\left(\lambda_{0} / N\right) \tilde{\mathbb{P}}$-a.s. by Assumption 2.3 (A1). The second term in the right hand side of (77) can be bounded from below (w.l.o.g. $\underline{\beta}\left(\lambda_{0} / N\right)<$ $1)$ :

$$
\begin{aligned}
& \frac{1}{\tilde{\mathbb{P}}\left[F^{N}\right]} \sum_{r=0}^{5} \tilde{\mathbb{E}}\left[\frac{1}{N} \mathbb{1}_{\tilde{F}^{N}} \sum_{\tau_{j} \in\left[\tilde{t}_{r}, \tilde{t}_{r+1}\right]} \log \beta_{j}\left(Z^{N, x^{N}}\left(\tau_{j}-\right)\right)\right]
\end{aligned}
$$

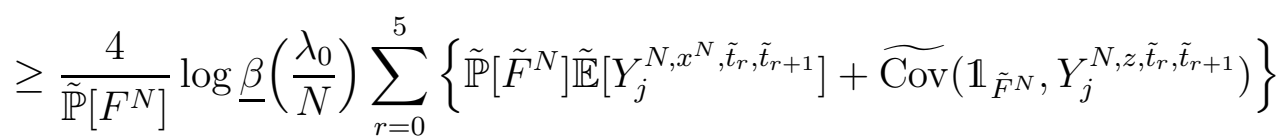

$$
\begin{aligned}
& \geq \frac{6 \tilde{\mathbb{P}}\left[\tilde{F}^{N}\right]}{\tilde{\mathbb{P}}\left[F^{N}\right]} \log \underline{\beta}\left(\frac{\lambda_{0}}{N}\right) \bar{\mu} \frac{\epsilon_{N}}{\lambda_{1}}+\frac{4}{\tilde{\mathbb{P}}\left[F^{N}\right]} \log \underline{\beta}\left(\frac{\lambda_{0}}{N}\right) \sqrt{\frac{\bar{\mu} \epsilon_{N}}{\lambda_{1}}} \sqrt{\delta\left(N, \epsilon_{N}\right)} \\
& \rightarrow 0
\end{aligned}
$$

as $N \rightarrow \infty$ by Assumption 2.3 (C) (cf. also (9)). For the first term in Equation (77), we compute for $j$ with $\mu_{j}>0$ (similarly as before, we assume w.l.o.g. that $\underline{\beta}(\tilde{\epsilon})<1$ and note that $\left.\beta_{j}\left(Z^{N, x^{N}}\left(\tau_{j}-\right)\right) \geq \underline{\beta}\left(\lambda_{1} \tilde{t}_{r}-\epsilon_{N}\right) \geq \underline{\beta}\left((r-4) \lambda_{1} \tilde{\Delta} / 2\right)\right)$

$$
\begin{aligned}
& \frac{1}{\tilde{\mathbb{P}}\left[F^{N}\right]} \sum_{r=6}^{\tilde{M}-1} \tilde{\mathbb{E}}\left[\frac{1}{N} \mathbb{1}_{\tilde{F}^{N}} \sum_{\tau_{j} \in\left[\tilde{t}_{r}, \tilde{t}_{r+1}\right]} \log \beta_{j}\left(Z^{N, x^{N}}\left(\tau_{j}-\right)\right)\right] \\
& \geq \frac{1}{\tilde{\mathbb{P}}\left[F^{N}\right]} \sum_{r=2}^{\tilde{M}-5} \log \underline{\beta}\left(r \lambda_{1} \tilde{\Delta} / 2\right) \tilde{\mathbb{E}}\left[\mathbb{1}_{\tilde{F}^{N}} Y^{\left.N, x^{N}, \tilde{t}_{r}, \tilde{t}_{r+1}\right]}\right. \\
& \quad=\frac{\tilde{\mathbb{P}}\left[\tilde{F}^{N}\right]}{\tilde{\mathbb{P}}\left[F^{N}\right]} \mu_{j}^{i} \sum_{r=2}^{\tilde{M}-5} \tilde{\Delta} \log \underline{\beta}\left(r \lambda_{1} \tilde{\Delta} / 2\right)+\frac{1}{\tilde{\mathbb{P}}\left[F^{N}\right]} \sum_{r=2}^{\tilde{M}-5} \log \underline{\beta}\left(r \lambda_{1} \tilde{\Delta} / 2\right) \widetilde{\operatorname{Cov}}\left(\mathbb{1}_{\tilde{F}^{N}}, Y^{\left.N, x^{N}, \tilde{t}_{r}, \tilde{t}_{r+1}\right) .}\right.
\end{aligned}
$$


For the first term in Equation (79), we have by Assumption 2.3 (C) (in particular by the fact that the integral below converges, cf. (12))

$$
\frac{\tilde{\mathbb{P}}\left[\tilde{F}^{N}\right]}{\tilde{\mathbb{P}}\left[F^{N}\right]} \mu_{j}^{i} \sum_{r=2}^{\tilde{M}-5} \tilde{\Delta} \log \underline{\beta}\left(r \lambda_{1} \tilde{\Delta} / 2\right) \rightarrow \mu_{j}^{i} \int_{0}^{2 \tilde{\epsilon} / \lambda_{1}} \log \underline{\beta}\left(\lambda_{1} \rho / 2\right) d \rho
$$

as $N \rightarrow \infty$. Similarly, we obtain for the second term in Equation (79),

$$
\begin{aligned}
& \frac{1}{\tilde{\mathbb{P}}\left[F^{N}\right]} \sum_{r=2}^{\tilde{M}-5} \log \underline{\beta}\left(r \lambda_{1} \tilde{\Delta} / 2\right) \widetilde{\operatorname{Cov}}\left(\mathbb{1}_{\tilde{F}^{N}}, Y^{N, x^{N}, \tilde{t}_{r}, \tilde{t}_{r+1}}\right) \\
& \quad \geq \frac{1}{\tilde{\mathbb{P}}\left[F^{N}\right]} \sqrt{\mu_{j}^{i} \frac{\delta\left(N, \epsilon_{N}\right)}{\tilde{\Delta}}} \sum_{r=2}^{\tilde{M}-5} \tilde{\Delta} \log \underline{\beta}\left(r \lambda_{1} \tilde{\Delta} / 2\right) \\
& \quad \rightarrow 0
\end{aligned}
$$

as $N \rightarrow 0$ by Assumption 2.3 (C) (cf. (8) and (12)).

Finally, we consider the third term in Equation (66). We obtain by Assumption (2.3) (C),

$$
\begin{aligned}
& \frac{1}{\tilde{\mathbb{P}}\left[F^{N}\right]} \sum_{j, \mu_{j}^{i}>0} \tilde{\mathbb{E}}\left[\frac{1}{N} \mathbb{1}_{F^{N} \backslash \tilde{F}^{N}} \sum_{\tau_{j} \in[0, \eta]} \log \beta_{j}\left(Z^{N, z}\left(\tau_{j}-\right)\right)\right] \\
& \geq \frac{1}{\tilde{\mathbb{P}}\left[F^{N}\right]} \log \underline{\beta}\left(\frac{\lambda_{0}}{N}\right) \sum_{j ; \mu_{j}^{i}>0}\left\{\tilde{\mathbb{P}}\left[\left(\tilde{F}^{N}\right)^{c}\right] \tilde{\mathbb{E}}\left[Y_{j}^{N, z, 0, \eta}\right]+\widetilde{\operatorname{Cov}}\left(\mathbb{1}_{\left(\tilde{F}^{N}\right)^{c}}, Y_{j}^{N, z, 0, \eta}\right)\right\} \\
& \geq \frac{1}{\tilde{\mathbb{P}}\left[F^{N}\right]} \log \underline{\beta}\left(\frac{\lambda_{0}}{N}\right) k \bar{\mu} \eta \delta\left(N, \epsilon_{N}\right)+\frac{1}{\tilde{\mathbb{P}}\left[F^{N}\right]} \log \underline{\beta}\left(\frac{\lambda_{0}}{N}\right) k \sqrt{\bar{\mu} \eta \delta\left(N, \epsilon_{N}\right)} \\
& \rightarrow 0
\end{aligned}
$$

as $N \rightarrow \infty$ similarly as before (cf. (8) and (9)) ).

We obtain by Equation (66) and (75), (77) - (82),

$$
\begin{aligned}
& \liminf _{N \rightarrow \infty} \frac{1}{\tilde{\mathbb{P}}\left[F^{N}\right]} \tilde{\mathbb{E}}\left[\frac{1}{N} \mathbb{1}_{F^{N}} \sum_{\tau \leq \eta} \log \beta_{j(\tau)}\left(Z^{N, x^{N}}(\tau-)\right)\right] \\
& \quad \geq \sum_{j=1}^{k} \mu_{j}^{i} \int_{2 \tilde{\epsilon} / \lambda_{1}}^{\eta}\left|\log \beta_{j}\left(\phi^{x}(t)\right)\right| d t-k \bar{\mu} \int_{0}^{2 \tilde{\epsilon} / \lambda_{1}}\left|\log \underline{\beta}\left(\lambda_{1} \rho / 2\right)\right| d \rho .
\end{aligned}
$$

We conclude by letting $\delta>0$ and choosing $\tilde{\epsilon}<\epsilon_{1}$ small enough such that (cf. Equations (55), (56) and Inequality (83); note that we require the convergence of the integral in (12) of Assumption 2.3 (C) here)

$$
\frac{\tilde{\epsilon}}{\lambda_{1}} k \bar{\mu}, k \bar{\mu} \int_{0}^{2 \tilde{\epsilon} / \lambda_{1}}\left|\log \beta_{j}\left(\phi^{x}(t)\right)\right| d t, k \bar{\mu} \int_{0}^{2 \tilde{\epsilon} / \lambda_{1}}\left|\log \underline{\beta}\left(\lambda_{1} \rho / 2\right)\right| d \rho<\frac{\delta}{4} .
$$

The assertion now follows from Inequality (154) and (55), (156), (157), (65) and (83)):

$$
\liminf _{N \rightarrow \infty} \tilde{\mathbb{E}}_{F^{N}}\left[\frac{X_{\eta}}{N}\right] \geq-\int_{0}^{\eta} \ell\left(\phi^{x}(t), \mu\right) d t-\delta .
$$

The uniformity of the convergence follows from the fact that we have used only Assumption $2.3(\mathrm{C})$ and Theorem 3.1, where the convergences are uniform in $x$. 
Again in the following result, the exponent $\alpha$ is the one from Assumption 2.3 (C).

Theorem 5.8. Assume that Assumption 2.3 holds. Let $x \in A$ and $x^{N} \in A^{N}$ such that

$$
\limsup _{N \rightarrow \infty}\left|x^{N}-x\right| N^{\alpha}<1
$$

Then, for $\phi \in D([0, T] ; A)$ and $\epsilon>0$,

$$
\liminf _{N \rightarrow \infty} \frac{1}{N} \log \mathbb{P}\left[\sup _{t \in[0, T]}\left|Z^{N, x^{N}}(t)-\phi(t)\right|<\epsilon\right] \geq-I_{T, x}(\phi) .
$$

Moreover the above convergence is uniform in $x \in A$

Proof. We can w.l.o.g. assume that $I_{T, x}(\phi) \leq K<\infty$. Let $\delta>0$ and divide the interval $[0, T]$ into $J$ subintervals as before. We define the function $\phi^{\eta}$ as before and choose $\eta_{1}$ small enough such that for all $\eta<\eta_{1}$ (cf. Lemma [5.6),

$$
\int_{\eta}^{T} L\left(\phi^{\eta}(t),\left(\phi^{\eta}\right)^{\prime}(t)\right) d t<\int_{0}^{T} L\left(\phi(t), \phi^{\prime}(t)\right) d t+\frac{\delta}{3} .
$$

We furthermore assume that $\eta<\eta_{1}$ is such that

$$
\sup _{t \in[0, T]}\left|\phi(t)-\phi^{\eta}(t)\right|<\frac{\epsilon}{4}
$$

Hence,

$\liminf _{N \rightarrow \infty} \frac{1}{N} \log \mathbb{P}\left[\sup _{t \in[0, T]}\left|Z^{N, x^{N}}(t)-\phi(t)\right|<\epsilon\right] \geq \liminf _{N \rightarrow \infty} \frac{1}{N} \log \mathbb{P}\left[\sup _{t \in[0, T]}\left|Z^{N, x^{N}}(t)-\tilde{\phi}^{\eta}(t)\right|<\frac{\epsilon}{2}\right]$.

From (43), for $t \geq \eta, \operatorname{dist}\left(\phi^{\eta}(t), \partial A\right) \geq \eta \lambda_{1}$. We define

$$
\begin{gathered}
\epsilon_{1}=\epsilon_{1}(\eta)=\frac{\epsilon}{2} \wedge \frac{\lambda_{1} \eta}{4} \\
\underline{\beta}^{\eta}:=\inf \left\{\beta_{j}(z) \mid 1 \leq j \leq k, z \in A, \operatorname{dist}(z, \partial A) \geq \frac{\eta \lambda_{1}}{2}\right\}>0
\end{gathered}
$$

and

$$
\tilde{\beta}_{j}^{\eta}(z):= \begin{cases}\beta_{j}(z) \vee \underline{\beta}^{\eta} & \text { if } z \in A \\ \tilde{\beta}_{j}^{\eta}\left(\psi_{A}(z)\right) & \text { else, }\end{cases}
$$

where the function $\psi_{A}$ has been specified in Assumption (A4). We denote by $\tilde{Z}^{N, z, \eta}$ the process starting at $z$ at time $\eta$ with rates $\tilde{\beta}_{j}^{\eta}$. As the $\log \tilde{\beta}_{j}^{\eta}$ are bounded, we have by Theorem 5.2 that there exists an

$$
\epsilon_{2}=\epsilon_{2}(\eta)<\epsilon_{1}(\eta)
$$

such that for all $\tilde{\epsilon}<\epsilon_{2}$,

$\liminf _{N \rightarrow \infty} \frac{1}{N} \log \left(\inf _{\left|z-\phi^{\eta}(\eta)\right|<\tilde{\epsilon}} \mathbb{P}\left[\sup _{t \in[\eta, T]}\left|\tilde{Z}^{N, z, \eta}(t)-\tilde{\phi}^{\eta}(t)\right|<\epsilon_{1}\right]\right) \geq-\int_{\eta}^{T} \tilde{L}^{\eta}\left(\phi^{\eta}(t),\left(\phi^{\eta}\right)^{\prime}(t)\right) d t-\frac{\delta}{3}$, 
where $\tilde{L}^{\eta}$ denotes the Legendre transform corresponding to the rates $\tilde{\beta}_{j}^{\eta}$. We readily observe that for all $t \in[\eta, T]$,

$$
\tilde{L}^{\eta}\left(\phi^{\eta}(t), \phi^{\eta}(t)\right)=L\left(\phi^{\eta}(t),\left(\phi^{\eta}\right)^{\prime}(t)\right)
$$

and that for $\left|z-\phi^{\eta}(\eta)\right|<\tilde{\epsilon}$, denoting by an abuse of notation $Z^{N, z}$ the process starting from $z$ at time $\eta$,

$$
\sup _{t \in[\eta, T]}\left|Z^{N, z}(t)-\phi^{\eta}(t)\right|<\epsilon_{1} \Leftrightarrow \sup _{t \in[\eta, T]}\left|\tilde{Z}^{N, z, \eta}(t)-\phi^{\eta}(t)\right|<\epsilon_{1} .
$$

and hence

$$
\begin{aligned}
\mathbb{P}\left[\sup _{t \in[\eta, T]}\left|Z^{N, z}(t)-\tilde{\phi}^{\eta}(t)\right|<\frac{\epsilon}{2}\right] & \geq \mathbb{P}\left[\sup _{t \in[\eta, T]}\left|Z^{N, z}(t)-\tilde{\phi}^{\eta}(t)\right|<\epsilon_{1}\right] \\
& =\mathbb{P}\left[\sup _{t \in[\eta, T]}\left|\tilde{Z}^{N, z, \eta}(t)-\tilde{\phi}^{\eta}(t)\right|<\epsilon_{1}\right]
\end{aligned}
$$

consequently for $\tilde{\epsilon}<\epsilon_{2}$

$$
\begin{aligned}
\liminf _{N \rightarrow \infty} \frac{1}{N} \log \left(\inf _{\left|z-\phi^{\eta}(\eta)\right|<\tilde{\epsilon}} \mathbb{P}\left[\sup _{t \in[\eta, T]}\left|Z^{N, z}(t)-\tilde{\phi}^{\eta}(t)\right|<\epsilon_{1}\right]\right) & \geq-\int_{\eta}^{T} L\left(\phi^{\eta}(t),\left(\phi^{\eta}\right)^{\prime}(t)\right) d t-\frac{\delta}{3} \\
& \geq-\int_{0}^{T} L\left(\phi(t), \phi^{\prime}(t)\right) d t-\frac{2 \delta}{3}
\end{aligned}
$$

where we have used (84) for the second inequality. We use the Markov property of $Z^{N}$ and obtain for $\tilde{\epsilon}<\epsilon_{2}$

$$
\begin{aligned}
& \mathbb{P}\left[\sup _{t \in[0, T]}\left|Z^{N, x^{N}}(t)-\phi(t)\right|<\epsilon\right] \geq \mathbb{P}\left[\sup _{t \in[0, \eta]}\left|Z^{N, x^{N}}(t)-\tilde{\phi}^{\eta}(t)\right|<\tilde{\epsilon}\right] \\
& \cdot \inf _{\left|z-\phi^{\eta}(\eta)\right|<\tilde{\epsilon}} \mathbb{P}\left[\sup _{t \in[\eta, T]}\left|Z^{N, z}(t)-\tilde{\phi}^{\eta}(t)\right|<\epsilon_{1}\right] .
\end{aligned}
$$

Combining the last two inequalities with Lemma 5.7, we deduce that ( $i$ being the index of the ball $B_{i}$ to which the starting point $x$ belongs)

$$
\begin{aligned}
\liminf _{N \rightarrow \infty} \frac{1}{N} \log \mathbb{P}\left[\sup _{t \in[0, T]}\left|Z^{N, x^{N}}(t)-\phi(t)\right|<\epsilon\right] & \geq-\int_{0}^{\eta} \ell\left(\phi^{x}(t), \mu^{i}\right) d t-\int_{0}^{T} L\left(\phi(t), \phi^{\prime}(t)\right) d t-\frac{2 \delta}{3} \\
& \geq-\int_{0}^{T} L\left(\phi(t), \phi^{\prime}(t)\right) d t-\delta
\end{aligned}
$$

thanks to Lemma 5.9 below, provided $\eta$ is small enough. The result follows since $\delta>0$ is arbitrary.

Lemma 5.9. Let $x \in B_{i}$, where $i \leq I_{1}$, and suppose $\phi^{x}(t)=x+t v_{i}$. Let moreover $\mu^{i}$ be such that $\sum_{j=1}^{k} \mu_{j}^{i} h_{j}=v_{i}$. Then, uniformly in $x$, as $t \rightarrow 0$,

$$
\int_{0}^{t} \ell\left(\phi^{x}(s), \mu^{i}\right) d s \rightarrow 0
$$


Proof. Since according to Assumption (A3) $d\left(\phi^{x}(t), \partial A\right) \geq \lambda_{1} t$, the result follows from (9) from Assumption (C).

Theorem 5.10. Assume that Assumption 2.3 as well as the assumptions from Theorem 5.8 hold. Then for any open set $G \subset D([0, T] ; A)$,

$$
\liminf _{N \rightarrow \infty} \frac{1}{N} \log \mathbb{P}\left[Z^{N, x^{N}} \in G\right] \geq-\inf _{\phi \in G} I_{T, x}(\phi) .
$$

Moreover the convergence is uniform in $x$.

Proof. The proof follows the same line of reasoning as the proof of Theorem 5.3 .

We will need the following stronger version. Recall the definition of $A^{N}$ at the start of section 2 ,

Theorem 5.11. Assume that Assumption 2.3 holds. Then for any open set $G \subset D([0, T] ; A)$ and any compact subset $K \subset A$,

$$
\liminf _{N \rightarrow \infty} \frac{1}{N} \log \inf _{x \in K \cap A^{N}} \mathbb{P}\left[Z^{N, x} \in G\right] \geq-\sup _{x \in K} \inf _{\phi \in G} I_{T, x}(\phi) .
$$

Proof. This follows readily from the uniformity in $x$ of the convergence in Theorem 5.10 .

\section{$6 \quad$ LDP upper bound}

We now prove the LDP upper bound. For reasons of readability, we split up the proof into four parts. In the first three parts, we prove the main auxiliary results required (Sections 6.1 - 6.3). Finally, we prove the main results of the section in Section 6.4.

In this section, whenever we consider the process $Z^{N, x}$, we will mean that the process $Z^{N}$ is started from the nearest point to $x$ on the grid $A^{N}$ (see the beginning of section 2 for the definition of $A^{N}$ ).

\subsection{Piecewise linear approximation}

The goal of this section is to prove that $Z^{N, x}$ is exponentially close to its piecewise linear approximation. For $Z^{N, x}$, we define the piecewise linear interpolation $Y^{N, x}$. To this end, we divide $[0, T]$ into $N$ subintervals $\left[t_{j-1}, t_{j}\right]$ with $t_{j}=\frac{j T}{N}, j=1, \ldots, N$. We define $t \in\left[t_{j-1}, t_{j}\right]$

$$
Y_{t}^{N, x}=Z^{N, x}\left(t_{j-1}\right)+\frac{t-t_{j-1}}{t_{j}-t_{j-1}}\left(Z^{N, x}\left(t_{j}\right)-Z^{N, x}\left(t_{j-1}\right)\right) .
$$

We prove that $Y^{N, x}$ is exponentially close to $Z^{N, x}$.

Lemma 6.1. Assume that $\beta_{j}(j=1, \ldots, k)$ is bounded. Let $\delta>0$. Then

$$
\limsup _{N \rightarrow \infty} \frac{1}{N} \log \mathbb{P}\left[\mathrm{d}\left(Y^{N, x}, Z^{N, x}\right)>\delta\right]=-\infty
$$

uniformly in $x \in A$. 
Proof. For any $1 \leq j \leq[N / T]$, we have the inclusion

$$
\left\{\sup _{t \in\left[t_{j-1}, t_{j}\right]}\left|Y_{t}^{N, x}-Z_{t}^{N, x}\right| \geq \delta\right\} \subset\left\{\sup _{t \in\left[t_{j-1}, t_{j}\right]}\left|Z_{t}^{N, x}-Z_{t_{j-1}}^{N, x}\right| \geq \delta / 2\right\} .
$$

It then follows from Lemma 3.6 that for some positive constant $C$ and for each $j$,

$$
\left.\mathbb{P}\left(\sup _{t \in\left[t_{j-1}, t_{j}\right]}\left|Y_{t}^{N, x}-Z_{t}^{N, x}\right| \geq \delta\right) \leq \exp (-C N \delta \log (C N \delta))\right) .
$$

Consequently

$$
\begin{aligned}
\mathbb{P}\left(\sup _{t \in[0, T]}\left|Y_{t}^{N, x}-Z_{t}^{N, x}\right| \geq \delta\right) & =\mathbb{P}\left(\bigcup_{j=1}^{[N / T]}\left\{\sup _{t \in\left[t_{j-1}, t_{j}\right]}\left|Y_{t}^{N, x}-Z_{t}^{N, x}\right| \geq \delta\right\}\right) \\
& \leq N \exp (-C N \delta \log (C N \delta))) .
\end{aligned}
$$

The result clearly follows.

\subsection{The modified rate function $I^{\delta}$}

In this section, we define a modified rate function $I^{\delta}$ and analyse how it relates to $I$. The main result is Corollary 6.6 below.

We define the following functional (Lemma 4.19 above). For $\delta>0, x \in A, y, \theta \in \mathbb{R}^{d}$, let

$$
\begin{aligned}
\tilde{\ell}_{\delta}(x, y, \theta) & :=\langle\theta, y\rangle-\sum_{j=1}^{k} \sup _{z=z^{j} \in A ;|z-x|<\delta} \beta_{j}(z)\left(\exp \left(\left\langle\theta, h_{j}\right\rangle\right)-1\right), \\
L_{\delta}(x, y) & :=\sup _{\theta \in \mathbb{R}^{d}} \tilde{\ell}_{\delta}(x, y, \theta) .
\end{aligned}
$$

Obviously, we have

$$
L_{\delta}(x, y) \leq L(x, y)
$$

and for the respectively defined functional, $I^{\delta}$,

$$
I^{\delta} \leq I .
$$

We obtain

$$
\begin{aligned}
L_{\delta}(x, y) & =\sup _{\theta \in \mathbb{R}^{d}}\left\{\langle\theta, y\rangle-\sum_{j=1}^{k} \sup _{z^{j} \in A ;\left|z^{j}-x\right|<\delta} \beta_{j}\left(z^{j}\right)\left(\exp \left(\left\langle\theta, h_{j}\right\rangle\right)-1\right)\right\} \\
& =\sup _{\theta \in \mathbb{R}^{d}} \inf _{z^{1}, \ldots, z^{k} \in A ;\left|z^{j}-x\right|<\delta}\left\{\langle\theta, y\rangle-\sum_{j=1}^{k} \beta_{j}\left(z^{j}\right)\left(\exp \left(\left\langle\theta, h_{j}\right\rangle\right)-1\right)\right\} \\
& =\inf _{z^{1}, \ldots, z^{k} \in A ;|z-x|<\delta} \sup _{\theta \in \mathbb{R}^{d}}\left\{\langle\theta, y\rangle-\sum_{j=1}^{k} \beta_{j}\left(z^{j}\right)\left(\exp \left(\left\langle\theta, h_{j}\right\rangle\right)-1\right)\right\} \\
& =\inf _{z^{1}, \ldots, z^{k},\left|z^{j}-x\right|<\delta} \inf _{\mu \in \tilde{V}_{z_{j}, y}} \sum_{j=1}^{k}\left(\beta_{j}\left(z^{j}\right)-\mu_{j}+\mu_{j} \log \mu_{j}-\mu_{j} \log \beta_{j}\left(z^{j}\right)\right)
\end{aligned}
$$




$$
=\ell\left(z^{*}, \mu^{*}\right)
$$

where we use the slight abuse of notation: for $z=\left(z^{1}, \ldots, z^{k}\right)$,

$$
\ell(z, \mu)=\sum_{j} \beta_{j}\left(z^{j}\right)-\mu_{j}+\mu_{j} \log \left(\frac{\mu_{j}}{\beta_{j}\left(z^{j}\right)}\right)
$$

Note that $\ell(x, \mu)$ depends on $x$ only through the rates $\beta(x)$.

Here, Equation (86) follows from Sion's min-max theorem, see e.g. Komiva [1988], and Equation (87) follows by Theorem 4.9, Equation (88) follows from Lemma 4.5 and the continuity of $\ell$ and $\mu^{*}$ (as a function in the state). We remark that $\left|z_{j}^{*}-x\right|=\delta$ is possible.

In a similar fashion as before (cf. Proposition 4.21), we define the sets

$$
\begin{aligned}
& \Phi^{\delta}(K):=\left\{\phi \in D([0, T] ; A) \mid I^{\delta}(\phi) \leq K\right\}, \\
& \Phi_{x}^{\delta}(K):=\left\{\phi \in D([0, T] ; A) \mid I_{x}^{\delta}(\phi) \leq K\right\} .
\end{aligned}
$$

In particular, we have $\Phi(K) \subset \Phi^{\delta}(K)$ and $\Phi_{x}(K) \subset \Phi_{x}^{\delta}(K)$ and $\Phi_{x}^{\delta}(K), \Phi^{\delta}(K)$ are increasing in $\delta$.

For technical reasons, we define for $m>0, z \in A$ the rates

$$
\beta_{j}^{m}(z):=\max \left\{\beta_{j}(z), 1 / m\right\}
$$

and the corresponding functionals $L^{m}$ and $I^{m}$ by replacing the rates $\beta_{j}$ by the rates $\beta_{j}^{m}$ in the respective definitions.

We will need the following slightly stronger version of Lemma [5.6, where again $\phi^{\eta}$ is defined from $\phi$ as in the lines before Lemma 5.6.

Lemma 6.2. Assume that Assumption 2.3 holds. Let $K>0$ and $\epsilon>0$. Then there exists an $\eta_{0}=\eta_{0}(T, K, \epsilon)>0$ such that for all $\eta<\eta_{0}$ there exists an $m_{0}>0$ such that for all $m>m_{0}$ and for all $\phi \in D([0, T] ; A)$ with $I_{T}^{m}(\phi) \leq K$,

$$
I_{T}\left(\phi^{\eta}\right)<K+\epsilon,
$$

where $\phi^{\eta}$ is defined before Lemma 5.6 and satisfies $\left\|\phi^{\eta}-\phi\right\| \leq \epsilon$.

Proof. We follows the first steps of the proof of Lemma Lemma5.1, where we replace $\mu^{*}(t)$ by $\mu^{m, *}(t)$ the optimal $\mu$ corresponding to $\left(\phi(t), \phi^{\prime}(t)\right)$ and jump rates $\beta_{j}^{m}$. Now (44) is replaced by

$$
L\left(\phi^{\eta}(t), \phi^{\prime}\left(t-\eta_{r}\right)\right) \leq \ell\left(\phi^{\eta}(t), \mu^{m, *}\left(t-\eta_{r}\right)\right) .
$$

We now choose $m>1 / \eta$ and deduce

$$
\left|\beta_{j}\left(\phi^{\eta}(t)\right)-\beta_{j}^{m}\left(\phi\left(t-\eta_{r}\right)\right)\right| \leq \frac{1}{m}+\left|\beta_{j}\left(\phi^{\eta}(t)\right)-\beta_{j}\left(\phi\left(t-\eta_{r}\right)\right)\right| \leq \delta_{K}^{\prime}(\eta),
$$

where $\delta_{K}^{\prime}(\eta)=\eta+\delta_{K}(\eta) \rightarrow 0$ as $\eta \rightarrow 0$ by the (uniform) continuity of the $\beta_{j}$. We deduce from (89) the following modified version of (46)

$L\left(\phi^{\eta}(t), \phi^{\prime}\left(t-\eta_{r}\right)\right)-L^{m}\left(\phi\left(t-\eta_{r}\right), \phi^{\prime}\left(t-\eta_{r}\right)\right) \leq k \delta_{K}^{\prime}(\eta)+\sum_{j} \mu^{m, *}\left(t-\eta_{r}\right) \log \frac{\beta_{j}^{m}\left(\phi\left(t-\eta_{r}\right)\right)}{\beta_{j}\left(\phi^{\eta}(t)\right)}$ 
since $\beta_{j}^{m}(\phi(t))>0$ and $\beta_{j}\left(\phi^{\eta}(t)\right)>0$ for $t \neq 0$.

We recall that $\lambda_{1}$ and the $v_{i}$ 's have been defined in Assumption 2.3 (A3), and that the $\tilde{v}_{i}{ }^{\prime}$ 's, $\bar{v}_{i}$ 's and $\hat{v}_{i}$ 's have been defined in the proof of Lemma 5.6.

By Assumption 2.3 (B4), there exists a constant $\lambda_{4}>0$ such that for $z \in B_{i_{r}}$ (and $\eta<\eta_{2} \leq \eta_{1}$ small enough, depending upon $\lambda_{1}$ and $\lambda_{2}$ but not on $\phi$, except through $K$ ),

$$
\beta_{j}(z)<\lambda_{4} \Rightarrow \beta_{j}\left(z+\eta \tilde{v}_{i_{r}}\right) \geq \beta_{j}(z) .
$$

We now want to bound from above the second term in the right hand side of (91). If $\beta_{j}\left(\phi\left(t-\eta_{r}\right)\right) \geq \lambda_{4}$, then $\beta_{j}\left(\phi\left(t-\eta_{r}\right)\right) \geq 1 / m$ and therefore $\beta_{j}^{m}\left(\phi\left(t-\eta_{r}\right)\right)=\beta_{j}\left(\phi\left(t-\eta_{r}\right)\right)$, so that the bound (48) holds.

Now consider the case $\beta_{j}\left(\phi\left(t-\eta_{r}\right)\right)<\lambda_{4}$. We define the function $s(\delta):=\inf \left\{\beta_{j}(x) \mid \mathrm{d}(x, \partial A) \geq\right.$ $\delta$; hence (recall the continuity of the $\beta_{j}$ and the compactness of $A$ ) $s(\delta)>0$ for $\delta>0$, and for $x \in A, \beta_{j}(x) \geq s(\mathrm{~d}(x, \partial A))$.

We furthermore let

$$
m_{0}=m_{0}\left(\eta, \lambda_{4}\right)>\max \left\{1 / \lambda_{4}, 1 / s\left(\lambda_{1} \eta\right)\right\}
$$

and recall that $\mathrm{d}\left(\phi^{\eta}(t), \partial A\right) \geq \lambda_{1} \eta$ for $t \geq \eta$ (cf. the discussion preceding Lemma 5.6) .

We let $m>m_{0}$. Since $\beta_{j}\left(\phi\left(t-\eta_{r}\right)\right)<\lambda_{4}$, by (92),

$$
\beta_{j}\left(\phi^{\eta}(t)\right) \geq \beta_{j}\left(\phi\left(t-\eta_{r}\right)\right) .
$$

By the definition of $s$, we have furthermore

$$
\left.\beta_{j}\left(\phi^{\eta}(t)\right)\right) \geq s\left(\lambda_{1} \eta\right) \geq 1 / m
$$

Combining these observations, we obtain

$$
\beta_{j}\left(\phi^{\eta}(t)\right) \geq \max \left\{\beta_{j}\left(\phi\left(t-\eta_{r}\right)\right), 1 / m\right\}=\beta_{j}^{m}\left(\phi\left(t-\eta_{r}\right)\right)
$$

and therefore

$$
\log \frac{\beta_{j}^{m}\left(\phi\left(t-\eta_{r}\right)\right)}{\beta_{j}\left(\phi^{\eta}(t)\right)} \leq 0 .
$$

From Lemma 4.3 and Lemma 4.5, there exist (universal, i.e., independent of $x$ and $m$ ) constants $B \geq B_{1} \vee B_{2}, B>1, C_{1}, C_{3}$ such that

$$
\begin{gathered}
|y|>B \Rightarrow \forall x \in A, m, L(x, y), L^{m}(x, y) \geq C_{1}|y| \log |y|, \\
|y|>B \Rightarrow \forall x \in A, m,\left|\mu^{m, *}\right|=\left|\mu^{*}(x, y, m)\right| \leq C_{3}|y| .
\end{gathered}
$$

Hence if $\left|\phi^{\prime}\left(t-\eta_{r}\right)\right| \geq B$, we get, instead of (52),

$$
\begin{aligned}
& L\left(\phi^{\eta}(t), \phi^{\prime}\left(t-\eta_{r}\right)\right)-L^{m}\left(\phi\left(t-\eta_{r}\right), \phi^{\prime}\left(t-\eta_{r}\right)\right) \\
& \quad \leq k \delta_{K}^{\prime}(\eta)+k C_{3}\left|\phi^{\prime}\left(t-\eta_{r}\right)\right| \frac{2 \delta_{K}(\eta)}{\lambda_{4}} \\
& \quad \leq k \delta_{K}^{\prime}(\eta)+k C_{3} \frac{2 \delta_{K}(\eta) L^{m}\left(\phi\left(t-\eta_{r}\right), \phi^{\prime}\left(t-\eta_{r}\right)\right)}{C_{1} \lambda_{4} \log \left|\phi^{\prime}\left(t-\eta_{r}\right)\right|} .
\end{aligned}
$$


If $\left|\phi^{\prime}\left(t-\eta_{r}\right)\right|<B$, Lemma 4.5 implies that $\left|\mu^{m, *}\left(t-\eta_{r}\right)\right| \leq \tilde{C} B$ for a universal constant $\tilde{C}>0$. Using Equations (90) and (91), we obtain

$$
L\left(\phi^{\eta}(t), \phi^{\prime}\left(t-\eta_{r}\right)\right)-L^{m}\left(\phi\left(t-\eta_{r}\right), \phi^{\prime}\left(t-\eta_{r}\right)\right) \leq k \delta_{K}^{\prime}(\eta)+k \tilde{C} B \frac{2 \delta_{K}(\eta)}{\lambda_{4}} .
$$

Inequalities (96) and (97) imply

$$
\begin{aligned}
& L\left(\phi^{\eta}(t), \phi^{\prime}\left(t-\eta_{r}\right)\right)-L^{m}\left(\phi\left(t-\eta_{r}\right), \phi^{\prime}\left(t-\eta_{r}\right)\right) \\
& \quad \leq k\left(\eta+\delta_{K}(\eta)\right)+k \tilde{C} B \frac{2 \delta_{K}(\eta)}{\lambda_{4}}+k C_{3} \frac{2 \delta_{K}(\eta) L^{m}\left(\phi\left(t-\eta_{r}\right), \phi^{\prime}\left(t-\eta_{r}\right)\right)}{C_{1} \lambda_{4} \log \left|\phi^{\prime}\left(t-\eta_{r}\right)\right|} \\
& \quad=: \delta_{1}(\eta)+\delta_{2}(\eta) L^{m}\left(\phi\left(t-\eta_{r}\right), \phi^{\prime}\left(t-\eta_{r}\right)\right)
\end{aligned}
$$

where $\delta_{i}(\eta) \rightarrow 0$ as $\eta \rightarrow 0, i=1,2$. We now choose $\eta>0$ such that

$$
\delta_{2}(\eta) K<\frac{\epsilon}{4} \quad \text { and } \quad T \delta_{1}(\eta)<\frac{\epsilon}{4}
$$

and choose $m>m_{0}(\eta)$; this yields

$$
I_{T}\left(\phi^{\eta}\right)<K+\epsilon
$$

In the following, we show a relation between $L^{m}$ and $L_{\delta}$.

Remark 6.3. It can easily be seen that Lemma 4.3 holds for $L_{\delta}$ (with exactly the same proof). The same holds true for Lemma 4.12 and Lemma 4.17.

Lemma 6.4. Let $\beta_{j}(j=1, \ldots, k)$ be bounded and $\epsilon>0$. Then there exists an $m_{0}>0$ such that for all $m>m_{0}$, there exists an $\delta_{0}>0$ such that for all $\delta<\delta_{0}$ and all $x \in A$ $y \in \mathbb{R}^{d}$,

$$
L^{m}(x, y) \leq \epsilon+(1+\epsilon) L_{\delta}(x, y)
$$

Proof. Let $m_{0}>0, m>m_{0}$ and $\delta>0$. We let $\mu^{*}=\mu^{*}\left(z^{*}, y\right)$ be the optimal $\mu$ associated to the optimal $z^{*}$ according to Equation (88). Then

$$
L^{m}(x, y)-L_{\delta}(x, y) \leq \ell^{m}\left(x, \mu^{*}\right)-\ell\left(z^{*}, \mu^{*}\right) .
$$

Furthermore, we have by the uniform continuity of the $\beta_{j}$ (cf. the proof of Lemma 6.2),

$$
\left|\beta_{j}^{m}(x)-\beta_{j}\left(z^{*}\right)\right| \leq \frac{1}{m}+K(\delta)=: K_{1}(m, \delta) .
$$

Moreover, we note that if $\beta_{j}(x)<\frac{1}{m}-K(\delta)$, then

$$
\log \frac{\beta_{j}\left(z^{*}\right)}{\beta_{j}^{m}(x)}<0
$$

On the other hand, if $\beta_{j}(x) \geq \frac{1}{m}-K(\delta)$, then

$$
\log \frac{\beta_{j}\left(z^{*}\right)}{\beta_{j}^{m}(x)} \leq \log \frac{\beta_{j}^{m}\left(z^{*}\right)}{\beta_{j}^{m}(x)} \leq \log \frac{\frac{1}{m}+K(\delta)}{\frac{1}{m}} \leq m K(\delta)=: K_{2}(m, \delta) .
$$


By Lemma 4.3 and Lemma 4.4, there exist constants $B, C_{1}, C_{3}$ and $C_{4}$ such that for all $x \in A$ and $y \in \mathcal{C}_{x}$,

$$
\begin{aligned}
L(x, y), L_{\delta}(x, y)>C_{1}|y| \log B & \text { if }|y|>B \\
\left|\mu^{*}(y)\right| \leq C_{3}|y| & \text { if }|y|>B \\
\left|\mu^{*}(y)\right| \leq C_{4} & \text { if }|y| \leq B
\end{aligned}
$$

(note that the constants in Inequality (102) are independent of $\delta$ ).

For $|y|>B$, we have by Inequalities (98), (99), (100), (101), (102) and (103),

$$
\begin{aligned}
L^{m}(x, y)-L_{\delta}(x, y) & \leq k K_{1}(m, \delta)+k C_{3}|y| K_{2}(m, \delta) \\
& \leq k K_{1}(m, \delta)+\frac{k C_{3} K_{2}(m, \delta)}{C_{1} \log B} L_{\delta}(x, y) .
\end{aligned}
$$

For $|y| \leq B$, we have by Inequalities (98), (199), (100), (101) and (104),

$$
L^{m}(x, y)-L_{\delta}(x, y) \leq k K_{1}(m, \delta)+k C_{4} K_{2}(m, \delta)
$$

The assertion now follows from Inequalities (105) and (106) by choosing $m_{0}$ large enough, $m>m_{0}$ and $\delta_{0}=\delta_{0}(m)$ small enough such that

$$
k K_{1}\left(m, \delta_{0}\right), k C_{4} K_{2}\left(m, \delta_{0}\right), \frac{k C_{3} K_{2}\left(m, \delta_{0}\right)}{C_{1} \log B}<\frac{\epsilon}{2} .
$$

We directly deduce the following result

Corollary 6.5. Let $\beta_{j}(j=1, \ldots, k)$ be bounded and continuous. For all $\epsilon, K, T>0$, there exists an $m_{0}>0$ such that for all $m>m_{0}$, there exists a $\delta_{0}>0$ such that for all $\delta<\delta_{0}$ and all functions $\phi$ with $I_{T}^{\delta}(\phi) \leq K-\epsilon$,

$$
I_{T}^{m}(\phi)<K
$$
[2005].

We now deduce from Lemma 6.2 and Corollary 6.5 the analog of Corollary 4.2 from Shwartz and Weis

Corollary 6.6. Assume that Assumption 2.3 holds. Then for all $\epsilon, K>0$, there exists a $\delta_{0}>0$ such that for all $\delta<\delta_{0}$,

$$
\Phi_{x}^{\delta}(K-\epsilon) \subset\left\{\phi \in D([0, T] ; A) \mid \mathrm{d}\left(\phi, \Phi_{x}(K)\right) \leq \epsilon\right\} .
$$

Proof. Let $\epsilon>0$ and choose $m_{0}, m, \delta_{0}, \delta$ according to Corollary 6.5 for $\epsilon / 2$. Let $\phi \underset{\sim}{\epsilon}$ $\Phi_{x}^{\delta}(K-\epsilon)$. Then by Corollary [6.5, $\phi \in \Phi_{x}^{m}(K-\epsilon / 2)$. By Lemma 6.2, there exists a $\tilde{\phi}$ such that

$$
\|\tilde{\phi}-\phi\|<\epsilon \quad \text { and } \quad I_{T, x}(\tilde{\phi}) \leq K .
$$




\subsection{Distance of $Y^{N}$ to $\Phi^{\delta}$}

In this section, we derive a result about the distance of $Y^{N}$, defined by (85), to $\Phi^{\delta}$ (Lemma 6.14 below).

We state the following elementary result (see, e.g., Roydon [1968], Chapter 3, Proposition 22).

Lemma 6.7. Let $f:[a, b] \rightarrow \mathbb{R}^{d}$ be measurable with $f \neq \infty$ almost everywhere. For all $\epsilon>0$, there exists a step function $g$ such that $|g-f|<\epsilon$ except on a set with measure less than $\epsilon$. Moreover the range of $g$ is a subset of the convex hull of the range of $f$.

We define for $\delta>0, \phi:[0, T] \rightarrow A$ and Borel-measurable $\theta:[0, T] \rightarrow \mathbb{R}^{d}$,

$$
I_{T}^{\delta}(\phi, \theta):=\int_{0}^{T} \tilde{\ell}_{\delta}\left(\phi(t), \phi^{\prime}(t), \theta(t)\right) d t .
$$

Lemma 6.8. Let $\log \beta_{j}(j=1, \ldots, k)$ be bounded. For all absolutely continuous $\phi$ : $[0, T] \rightarrow A$ with $I_{T}^{\delta}(\phi)<\infty$ and all $\epsilon>0$ there exists a step function $\theta:[0, T] \rightarrow \mathbb{R}^{d}$ such that

$$
I_{T}^{\delta}(\phi, \theta) \geq I_{T}^{\delta}(\phi)-\epsilon .
$$

Proof. As $I^{\delta}(\phi)<\infty$, there exists a large enough positive number $B$ such that

$$
\int_{0}^{T} \mathbb{1}_{\left\{\left|\phi^{\prime}(t)\right|>B\right\}} L_{\delta}\left(\phi^{\prime}(t), \phi(t)\right) d t \leq \frac{\epsilon}{3}
$$

(cf. Lemma 4.17 and Remark 6.3). We set

$$
\theta_{1}(t):=0 \quad \text { if }\left|\phi^{\prime}(t)\right|>B .
$$

By Lemma 4.12 (which holds true with $L$ replaced by $L_{\delta}$, see Remark 6.3 - this is where we need the assumption that the $\log \beta_{j}$ are bounded), there exists a constant $\tilde{B}$ such that for all $x \in A$ and $y \in \mathcal{C}$ with $|y| \leq B$,

$$
\sup _{|\theta| \leq \tilde{B}} \tilde{\ell}_{\delta}(x, y, \theta)>L_{\delta}(x, y)-\frac{\epsilon}{6 T} .
$$

We set

$$
D:=\{(x, y, \theta)|x \in A, y \in \mathcal{C},| y|\leq B,| \theta \mid \leq \tilde{B}\} .
$$

The function $\tilde{\ell}_{\delta}$ is uniformly continuous on $D$. Hence there exists an $\eta>0$ such that for $|x-\tilde{x}|,|y-\tilde{y}|,|\theta-\tilde{\theta}|<\eta$,

$$
\left|\tilde{\ell}_{\delta}(x, y, \theta)-\tilde{\ell}_{\delta}(\tilde{x}, \tilde{y}, \tilde{\theta})\right|<\frac{\epsilon}{6 T} .
$$

By a compactness argument, we obtain a finite cover $\left\{\theta_{i, j}, x_{i}, y_{j}\right\}$ of $D$ such that

$$
\tilde{\ell}_{\delta}\left(x_{i}, y_{j}, \theta_{i, j}\right) \geq L_{\delta}(x, y)-\frac{\epsilon}{3 T} \text { for }\left|x-x_{i}\right|,\left|y-y_{j}\right|<\eta .
$$

We set

$$
\theta_{1}(t):=\theta_{i, j} \quad \text { if }\left|\phi(t)-x_{i}\right|,\left|\phi^{\prime}(t)-y_{j}\right|<\eta
$$


(with some kind of tie-breaking rule). Hence $\theta_{1}$ only takes finitely many values. However, it is not clear whether $\theta_{1}$ is piecewise constant.

We now choose $\tilde{\eta}$ small enough such that Leb $[E]<\frac{\tilde{\eta}}{2}$ implies that

$$
\int_{[0, T] \cap E} L_{\delta}\left(\phi(t), \phi^{\prime}(t)\right) d t \vee \int_{[0, T] \cap E} \sup _{|\theta| \leq \tilde{B}}\left(-\tilde{\ell}_{\delta}\left(\phi(t), \phi^{\prime}(t), \theta\right)\right) d t<\frac{\epsilon}{3} .
$$

and

$$
\min \left|\theta_{i, j}-\theta_{l, m}\right|>\tilde{\eta}, \quad \min \left|\theta_{i, j}\right|>\tilde{\eta} .
$$

By Lemma 6.7, there exists a step function $\theta_{2}$ with $\left|\theta_{1}-\theta_{2}\right|<\frac{\tilde{\eta}}{2}$ except on a set $\tilde{E}$ with Lebesgue measure $<\frac{\tilde{\eta}}{2}$.

Hence there exists a step function $\theta$ which agrees with $\theta_{1}$ except on $\tilde{E}$ (modify $\theta_{2}$ if necessary such that $\left|\theta_{1}-\theta_{2}\right|<\frac{\tilde{\eta}}{2} \Rightarrow \theta_{2}=\theta_{1}$ on $\tilde{E}^{c}$. Note that $|\theta(t)| \leq \tilde{B}$, for all $t \in[0, T]$.

We conclude by collecting the approximations above:

$$
\begin{aligned}
& I_{T}^{\delta}(\phi)=\int_{[0, T]} L_{\delta}\left(\phi(t), \phi^{\prime}(t)\right) d t \\
& \leq \int_{[0, T] \cap\left\{\left|\phi^{\prime}(t)\right|>B\right\}} L_{\delta}\left(\phi(t), \phi^{\prime}(t)\right) d t+\int_{[0, T] \cap \tilde{E}} L_{\delta}\left(\phi(t), \phi^{\prime}(t)\right) d t \\
& +\int_{[0, T] \cap\left(\left\{\left|\phi^{\prime}(t)\right| \leq B\right\} \cup \tilde{E}^{c}\right)} L_{\delta}\left(\phi(t), \phi^{\prime}(t)\right) d t \\
& \leq \frac{2 \epsilon}{3}+\int_{[0, T] \cap\left(\left\{\mid \phi^{\prime}(t) \leq B\right\} \cup \tilde{E}^{c}\right)} \tilde{\ell}_{\delta}\left(\phi(t), \phi^{\prime}(t), \theta(t)\right) d t \\
& =\frac{2 \epsilon}{3}+\int_{[0, T]} \tilde{\ell}_{\delta}\left(\phi(t), \phi^{\prime}(t), \theta(t)\right) d t-\int_{[0, T] \cap \tilde{E}} \tilde{\ell}_{\delta}\left(\phi(t), \phi^{\prime}(t), \theta(t)\right) d t-\int_{[0, T] \cap\left\{\left|\phi^{\prime}(t)\right|>B\right\} \cap \tilde{E}^{c}} \tilde{\ell}_{\delta}\left(\phi(t), \phi^{\prime}(t), \theta(t)\right) d t \\
& \leq \epsilon+\int_{[0, T]} \tilde{\ell}_{\delta}\left(\phi(t), \phi^{\prime}(t), \theta(t)\right) d t
\end{aligned}
$$

Indeed $\theta(t)=0$ on the set $\left\{\left|\phi^{\prime}(t)\right|>B\right\} \cap \tilde{E}^{c}$, while (109) implies that the second integral in the next to last line is bounded by $\epsilon / 3$.

We next prove.

Lemma 6.9. Let $u:[0, T] \rightarrow \mathbb{R}$ be nonnegative and absolutely continuous and $\delta>0$. Then there exists an $\eta>0$, a Borel set $E \subset[0, T]$ with $\operatorname{Leb}(E)<\delta$ and two finite collections $\left(J_{i}\right)_{i \in \mathcal{I}_{+}}$and $\left(H_{j}\right)_{j \in \mathcal{I}_{0}}$ of subintervals of $[0, T]$ such that

$$
[0, T]=E \cup \bigcup_{i \in \mathcal{I}_{+}} J_{i} \cup \bigcup_{j \in \mathcal{I}_{0}} H_{j}
$$

and for all $i \in \mathcal{I}_{+}, j \in \mathcal{I}_{0}$,

$$
\inf _{t \in J_{i}} u(t)>\eta, \quad u(t)=0 \text { on } H_{j} \cap E^{c} .
$$

Proof. Given $t \in[0, T]$ such that $u(t)>0$, let $\mathcal{O}_{t}$ be the largest open interval containing $t$ such that $u(s)>0$ for all $s \in \mathcal{O}_{t}$. Let $m_{t}=\max \left\{u(t), t \in \mathcal{O}_{t}\right\}$. Since $u$ is absolutely 
continuous, there is a finite number of intervals $\mathcal{O}_{t}$ such that $m_{t}>1 / m$, for each $m \geq 1$. Hence there are at most countably many open intervals $\left\{\mathcal{O}_{i}, i \geq 1\right\}$ where $u$ is positive. Choose $M$ large enough such that

$$
\operatorname{Leb}\left(\cup_{i=M+1}^{\infty} \mathcal{O}_{i}\right) \leq \frac{\delta}{2}
$$

For $1 \leq i \leq M$, let $J_{i} \subset \mathcal{O}_{i}$ be a closed interval such that

$$
\operatorname{Leb}\left(\mathcal{O}_{i} \backslash J_{i}\right) \leq \frac{\delta}{2 M}
$$

Let

$$
E=\left(\cup_{i=M+1}^{\infty} \mathcal{O}_{i}\right) \cup\left(\cup_{i=1}^{M} \mathcal{O}_{i} \backslash J_{i}\right) .
$$

Clearly $\operatorname{Leb}(E) \leq \delta$. Let $\bar{M}$ be the number of connected components of $[0, T] \backslash \cup_{i=1}^{M} J_{i}$. For $1 \leq j \leq \bar{M}$, let $H_{j}$ denote the closure of the $j$-th connected component of $[0, T] \backslash \cup_{i=1}^{M} J_{i}$. $H_{j}$ is an interval. Moreover

$$
\begin{gathered}
\inf _{1 \leq i \leq M} \inf _{t \in J_{i}} u(t)=\eta>0, \quad \text { and } \\
u(t)=0, \quad \text { if } t \in H_{j} \cap E^{c} .
\end{gathered}
$$

We require this result for the proof of Lemma 4.6 of Shwartz and Weiss [2005]. This is a (more general) variant of Lemma 5.43 of Shwartz and Weiss [1995] (cf. also Lemma 6.8).

Lemma 6.10. Assume that $\beta_{j}(j=1, \ldots, k)$ is bounded and Lipschitz continuous. Then for all $\phi$ with $I_{T}(\phi)<\infty$ and $\epsilon>0$, there exists a step function $\theta$ such that

$$
I_{T}^{\delta}(\phi, \theta) \geq I_{T}^{\delta}(\phi)-\epsilon .
$$

Proof. If none of the $\beta_{j}(\phi(t))$ vanishes on the interval $[0, T]$, then the proof of Lemma 6.8 applies. If that is not the case, we note that since $\phi$ is absolutely continuous and $\beta_{j}$ is Lipschitz continuous, $t \rightarrow \beta_{j}(\phi(t))$ is absolutely continuous. Hence we can apply Lemma 6.9 to the function $u(t):=\beta_{j}(\phi(t))$, and associate to each $1 \leq j \leq k$ intervals $\left(J_{i}^{j}\right)_{i \in \mathcal{I}_{+}}$ and $\left(H_{i}^{j}\right)_{i \in \mathcal{I}_{0}}$. It is not hard to see that to each $\eta>0$ one can associate a real $\eta>0$, an integer $M$, a collection $\left(I_{i}\right)_{1 \leq i \leq M}$ of subintervals of $[0, T]$, with the following properties

$$
[0, T]=E \cup \bigcup_{1 \leq i \leq M} I_{i}
$$

with $\operatorname{Leb}(E) \leq \delta$, and moreover to each $1 \leq i \leq N$ we can associate a subset $\mathcal{A} \subset$ $\{1,2, \ldots, k\}$ such that

$$
\beta_{j}(\phi(t))>\eta, \quad \text { if } j \in \mathcal{A}, t \in I_{i}, \quad \text { and } \beta_{j}(\phi(t))=0 \text {, if } j \notin \mathcal{A}, t \in I_{i} \cap E^{c} .
$$

Each interval $I_{i}$ is an intersection of $J_{i}^{j}$ 's for $j \in \mathcal{A}$ and of $H_{i}^{j}$ 's for $j \notin \mathcal{A}$.

On each subinterval $I_{i}$, by considering the process with rates and jump directions $\left\{\beta_{j}, h_{j}, j \in \mathcal{A}\right\}$ only, we can deduce from Lemma 6.8 that there exists a step function $\theta$ such that

$$
\int_{I_{i}} \tilde{\ell}_{\delta}\left(\phi(t), \phi^{\prime}(t), \theta(t)\right) \geq \int_{I_{i}} L_{\delta}\left(\phi(t), \phi^{\prime}(t)\right) d t-\frac{\epsilon}{2 M} .
$$


In fact there exists a unique stepfunction $\theta$ defined on $[0, T]$, such that each of the above inequality holds and moreover, by the same argument as in the proof of Lemma 6.8. provided $\eta$ is small enough,

$$
\int_{E} \tilde{\ell}_{\delta}\left(\phi(t), \phi^{\prime}(t), \theta(t)\right) \geq-\frac{\epsilon}{2}
$$

The result follows.

We now define for $M \in \mathbb{N}$

$$
\mathcal{K}(M):=\bigcap_{m \geq M}\left\{\phi \in C([0, T] ; A) \mid V_{2^{-m}}(\phi) \leq \frac{1}{\log m}\right\},
$$

where $V_{\delta}$ is the modulus of continuity:

$$
V_{\delta}(\phi):=\sup _{s, t \in[0, T],|s-t|<\delta}|\phi(s)-\phi(t)| .
$$

We readily observe (Arzelà-Ascoli) that $\mathcal{K}(M)$ is compact in $C\left([0, T] ; \mathbb{R}^{d}\right)$.

We next obtain exponential tightness for the sequence $Y^{N, x}$ defined in (855).

Lemma 6.11. Assume that $\beta_{j}(j=1, \ldots, k)$ is bounded. There exists a positive constant a such that for all $M$ large enough and for all $x \in A$,

$$
\limsup _{N \rightarrow \infty} \frac{1}{N} \log \mathbb{P}\left[Y^{N, x} \notin \mathcal{K}(M)\right] \leq-a \frac{M}{\log M} .
$$

Proof. Suppose that

$$
V_{2^{-m}}\left(Y^{N}\right) \leq \frac{1}{\log m}, \text { for } m=M, \ldots, M(N), \text { where } M(N)=\left\lceil\frac{\log (N / T)}{\log 2}\right\rceil .
$$

It is plain that $m \geq M(N)$ implies that $2^{-m}<T / N$, hence $V_{2^{-(m+1)}}\left(Y^{N}\right)=\frac{1}{2} V_{2^{-m}}\left(Y^{N}\right)$. Then, provided $N>4 T, M(N) \geq 2$, hence for any $m \geq M(N), m+1 \leq m^{2}$, and also $(2 \log m)^{-1} \leq(\log (m+1))^{-1}$, and it follows that (110) implies that $Y^{N, x} \in \mathcal{K}(M)$.

Now if $M \leq m \leq M(N)$,

$$
\left\{V_{2^{-m}}\left(Y^{N}\right)>\frac{1}{\log m}\right\} \subset \bigcup_{j=0}^{N-1}\left\{\sup _{t_{j} \leq s \leq t_{j}+2^{1-m}}\left|Z_{s}^{N, x}-Z_{t_{j}}^{N, x}\right|>\frac{1}{2 \log m}\right\} .
$$

Consequently, with the help of Lemma [3.6, for some $C>0$ and provided $M$ is large enough,

$$
\begin{aligned}
\mathbb{P}\left[Y^{N, x} \notin \mathcal{K}(M)\right] & \leq \sum_{m=M}^{M(N)} \mathbb{P}\left(V_{2^{-m}}\left(Y^{N}\right)>\frac{1}{\log m}\right) \\
& \leq N \sum_{m=M}^{M(N)} \exp \left(-\frac{C N}{\log m} \log \left(\frac{C 2^{m}}{\log m}\right)\right) \\
& \leq M(N) N \exp \left(-\frac{C N}{\log M} \log \left(\frac{C 2^{M}}{\log M}\right)\right)
\end{aligned}
$$


where the last inequality follows from the fact that for $x>0$ large enough, the mapping $x \rightarrow(\log x)^{-1} \log \left(\frac{C 2^{x}}{\log x}\right)$ is increasing. Consequently

$$
\frac{1}{N} \log \mathbb{P}\left[Y^{N, x} \notin \mathcal{K}(M)\right] \leq \frac{\log M(N)}{N}+\frac{\log N}{N}-\frac{c}{\log M} \log \left(\frac{C 2^{M}}{\log M}\right) .
$$

It remains to take the limsup as $N \rightarrow \infty$.

We now establish the main local estimate for $Y^{N}$.

Lemma 6.12. Assume that $\beta_{j}(j=1, \ldots, k)$ is bounded. For all $\delta>0$, we have uniformly in $x \in A$ and $\theta$ in a bounded set,

$$
\limsup _{N \rightarrow \infty} \log \mathbb{E}\left[\exp \left(N\left\langle Y^{N, x}\left(\frac{T}{N}\right)-Y^{N, x}(0), \theta\right\rangle\right)\right] \leq T \cdot \sum_{j=1}^{k} \sup _{z^{j} \in A,\left|z^{j}-x\right| \leq \delta} \beta_{j}\left(z^{j}\right)\left(\mathrm{e}^{\left\langle\theta, h_{j}\right\rangle}-1\right) .
$$

Proof. It is not hard to verify that for any $\theta \in \mathbb{R}^{d}$, the process

$$
M_{t}^{\theta}:=\exp \left(N\left\langle Z_{t}^{N, x}-x, \theta\right\rangle-N \sum_{j=1}^{k}\left(e^{\left\langle\theta, h_{j}\right\rangle}-1\right) \int_{0}^{t} \beta_{j}\left(Z^{N, x}(s)\right) d s\right)
$$

is a martingale with $M_{0}^{\theta}=1$, hence $\mathbb{E}\left[M_{t}^{\theta}\right]=1$. Let

$$
\left.S_{N, \delta}:=\left\{\sup _{0 \leq t \leq T / N} \mid Z_{t}^{N, x}-x\right] \leq \delta\right\} .
$$

Since $M_{t}^{\theta}>0, \mathbb{E}\left[M_{T / N}^{\theta} \mathbf{1}_{S_{N, \delta}}\right] \leq 1$. But on the event $S_{N, \delta}$,

$$
M_{T / N}^{\theta} \geq \exp \left(N\left\langle Z^{N, x}(T / N)-x, \theta\right\rangle-T \sum_{j=1}^{k} \sup _{z^{j} \in A,\left|z^{j}-x\right| \leq \delta} \beta_{j}\left(z^{j}\right)\left(e^{\left\langle\theta, h_{j}\right\rangle}-1\right)\right),
$$

hence

$$
\mathbb{E}\left[\exp \left(N\left\langle Z^{N, x}(T / N)-x, \theta\right\rangle\right) \mathbf{1}_{S_{N, \delta}}\right] \leq \exp \left(T \sum_{j=1}^{k} \sup _{z^{j} \in A,\left|z^{j}-x\right| \leq \delta} \beta_{j}\left(z^{j}\right)\left(e^{\left\langle\theta, h_{j}\right\rangle}-1\right)\right) .
$$

On the other hand, from Lemma 3.6, for some $C>0$, whenever $|\theta| \leq B$,

$$
\begin{aligned}
\mathbb{E}\left[\exp \left(N\left\langle Z^{N, x}(T / N)-x, \theta\right\rangle\right) \mathbf{1}_{S_{N, \delta}^{c}}\right] & \leq \sum_{\ell=1}^{\infty} e^{N(\ell+1) \delta|\theta|} \mathbb{P}\left(\ell \delta \leq\left|Z^{N, x}(T / N)-x\right| \leq(\ell+1) \delta\right) \\
& \leq \sum_{\ell=1}^{\infty} \exp (N \delta[(\ell+1) B-C \ell \log (C N \ell \delta)]) \\
& \leq \sum_{\ell=1}^{\infty} a(N, \delta)^{\ell} \\
& \leq 2 a(N, \delta),
\end{aligned}
$$


provided $N$ is large enough, such that

$$
a(N, \delta):=\exp (N \delta[2 B-C \log (C N \delta)]) \leq 1 / 2 .
$$

Finally

$$
\mathbb{E}\left[\exp \left(N\left\langle Z^{N, x}(T / N)-x, \theta\right\rangle\right)\right] \leq \exp \left(T \sum_{j=1}^{k} \sup _{z^{j} \in A,\left|z^{j}-x\right| \leq \delta} \beta_{j}\left(z^{j}\right)\left(e^{\left\langle\theta, h_{j}\right\rangle}-1\right)\right)+2 a(N, \delta) .
$$

The result follows from the fact that $a(N, \delta) \rightarrow 0$ as $N \rightarrow \infty$, for any $\delta>0$.

We next establish

Lemma 6.13. Let $\beta_{j}(j=1, \ldots, k)$ be bounded and continuous. Let $\theta:[0, T] \rightarrow \mathbb{R}^{d}$ be a step function, $\delta>0$ and $\mathcal{K} \subset \mathcal{K}(M)$ be a compact set, such that the subset $\mathcal{K}^{a c}$ consisting of those elements of $\mathcal{K}$ which are absolutely continuous is dense in $\mathcal{K}$. Then

$$
\limsup _{N \rightarrow \infty} \frac{1}{N} \log \mathbb{P}\left[Y^{N, x} \in \mathcal{K}\right] \leq-\inf _{\phi \in \mathcal{K}, \phi(0)=x} I^{\delta}(\phi, \theta)
$$

uniformly in $x$.

Proof. Let $\theta$ be a fixed step function from $[0, T]$ into $\mathbb{R}^{d}$, which we assume w.l.o.g. to be right continuous, and let $\mathcal{K}$ be a given compact subset of $C\left([0, T] ; \mathbb{R}^{d}\right)$, which has the property that $\mathcal{K}^{a c}$ is dense in $\mathcal{K}$. We define for each $\delta>0$ the mapping $g_{\delta}: \mathbb{R}^{2 d} \rightarrow \mathbb{R}$ by

$$
g_{\delta}(z, \theta)=\sum_{j=1}^{k} \sup _{\left|z_{j}-x\right| \leq \delta} \beta_{j}\left(z_{j}\right)\left(e^{\left\langle\theta, h_{j}\right\rangle}-1\right) .
$$

We let $t_{\ell}:=\ell T / N$, and define for $z \in \mathcal{K}^{a c}$, the two quantities

$$
\begin{aligned}
\tilde{S}_{N}(z, \theta) & =\sum_{\ell=1}^{N}\left\langle z\left(t_{\ell}\right)-z\left(t_{\ell-1}\right), \theta\left(t_{\ell-1}\right)\right\rangle-\frac{T}{N} \sum_{\ell=1}^{N} g_{\delta}\left(z\left(t_{\ell-1}\right), \theta\left(t_{\ell-1}\right)\right), \\
S(z, \theta) & =\int_{0}^{T}\left\langle z^{\prime}(t), \theta(t)\right\rangle d t-\frac{T}{N} \sum_{\ell=1}^{N} g_{\delta}\left(z\left(t_{\ell-1}\right), \theta\left(t_{\ell-1}\right)\right) .
\end{aligned}
$$

Choose any $\eta>0$. We can assume that $N_{0}$ has been chosen large enough, such that

$$
\sup _{z \in \mathcal{K}^{a c}}\left|\tilde{S}_{N}(z, \theta)-S(z, \theta)\right| \leq \eta
$$

Indeed, this difference is bounded by twice the number of jumps of $\theta$ times the sup of $|\theta(t)|$, times the maximal oscillation of $z$ on intervals of length $1 / N$ in $[0, T]$.

It follows from Lemma 6.12 and the Markov property that, provided $N_{0}$ has been chosen large enough, for any $N \geq N_{0}$,

$$
\mathbb{E}\left[\exp \left(N \tilde{S}_{N}\left(Y^{N, x}, \theta\right)\right)\right] \leq \exp (N \eta)
$$


Clearly, on the event $Y^{N, x} \in \mathcal{K}$,

$$
\exp \left[N\left(\tilde{S}_{N}\left(Y^{N}, \theta\right)-\inf _{z \in \mathcal{K}^{a c}} \tilde{S}_{N}(z, \theta)\right)\right] \geq 1,
$$

and combining this fact with the previous inequalities, we deduce that

$$
\begin{aligned}
\mathbb{P}\left(Y^{N, x} \in \mathcal{K}\right) & \leq \mathbb{E} \exp \left[N\left(\tilde{S}_{N}\left(Y^{N, x}, \theta\right)-\inf _{z \in \mathcal{K}^{a c}} \tilde{S}_{N}(z, \theta)\right)\right] \\
& \leq \exp (N \eta) \exp \left(-N \inf _{z \in \mathcal{K}^{a c}} \tilde{S}_{N}(z, \theta)\right) \\
& \leq \exp (2 N \eta) \exp \left(-N \inf _{z \in \mathcal{K}^{a c}} S_{N}(z, \theta)\right)
\end{aligned}
$$

Now, uniformly in $z \in \mathcal{K}^{a c}, S_{N}(z, \theta) \rightarrow I^{\delta}(z, \theta)$, where

$$
I^{\delta}(z, \theta)=\int_{0}^{T}\left\langle z^{\prime}(t), \theta(t)\right\rangle d t-\int_{0}^{T} g_{\delta}(z(t), \theta(t)) d t .
$$

The result follows from the last two facts, since $\eta>0$ can be chosen arbitrarily small, and $\mathcal{K}^{a c}$ is dense in $\mathcal{K}$.

We now have

Lemma 6.14. Assume that $\beta_{j}(j=1, \ldots, k)$ is bounded and Lipschitz continuous. Then for all $K>0, \delta>0$ and $\epsilon>0$,

$$
\limsup _{N \rightarrow \infty} \frac{1}{N} \log \mathbb{P}\left[\mathrm{d}\left(Y^{N, x}, \Phi_{x}^{\delta}(K)\right)>\epsilon\right] \leq-K+\epsilon
$$

uniformly in $x \in A$.

Proof. We fix $\epsilon, \delta, K>0$ and choose $M \in \mathbb{N}$ such that $a \frac{M}{\log M}>K-\epsilon$, where $a$ is the constant appearing in Lemma 6.12

For absolute continuous $\phi:[0, T] \rightarrow A$ with $I^{\delta}(\phi)<\infty$, there exists a step function $\theta^{\phi}$ such that

$$
I^{\delta}\left(\phi, \theta^{\phi}\right) \geq I^{\delta}(\phi)-\frac{\epsilon}{2}
$$

(cf. Lemma 6.8). It can easily be verified by elementary calculus that the function $I^{\delta}\left(\cdot, \theta^{\phi}\right)$ is continuous for the sup norm topology on the set of absolutely continuous functions. Hence there exists a number $0<\eta^{\phi}<\frac{\epsilon}{2}$ such that for all absolutely continuous $\tilde{\phi}$ with $\|\phi-\tilde{\phi}\|<\eta^{\phi}$

$$
I^{\delta}\left(\tilde{\phi}, \theta^{\phi}\right) \geq I^{\delta}(\phi)-\epsilon
$$

We consider the compact set

$$
\mathcal{K}^{x}(M):=\{\phi \in \mathcal{K}(M) \mid \phi(0)=x\}
$$

(cf. the definition preceding Lemma 6.11). By a compactness argument, there exist finitely many absolutely continuous functions $\left\{\phi_{i}, 1 \leq i \leq m\right\} \subset \mathcal{K}^{x}(M)$ with $I^{\delta}\left(\phi_{i}\right)<\infty$ (and corresponding $\theta_{i}:=\theta^{\phi_{i}}$ and $\eta_{i}:=\eta^{\phi_{i}}$ ) such that

$$
\mathcal{K}^{x}(M) \subset \bigcup_{i=1}^{m} B_{\eta_{i}}\left(\phi_{i}\right) .
$$


For each $1 \leq i \leq m$, we define the compact set

$$
\mathcal{K}_{i}^{x}(M):=\overline{B_{\eta_{i}}\left(\phi_{i}\right) \cap \mathcal{K}^{x}(M)} .
$$

We now let

$$
\mathcal{I}:=\left\{1 \leq i \leq m \mid \mathrm{d}\left(\phi_{i}, \Phi_{x}^{\delta}(K)\right) \geq \eta_{i}\right\} .
$$

Then $\mathrm{d}\left(Y^{x, N}, \Phi_{x}^{\delta}(K)\right) \geq \epsilon$ and $Y^{x, N} \in \mathcal{K}_{i}^{x}(M)$ imply $i \in \mathcal{I}$, since $\eta_{i} \leq \epsilon / 2$. Hence

$$
\begin{aligned}
\limsup _{N \rightarrow \infty} & \frac{1}{N} \log \mathbb{P}\left[\mathrm{d}\left(Y^{x, N}, \Phi_{x}^{\delta}(K)\right) \geq \epsilon\right] \\
& \leq \limsup _{N \rightarrow \infty} \frac{1}{N} \log \left\{\mathbb{P}\left[Y^{x, N} \notin \mathcal{K}^{x}(M)\right]+\sum_{i \in \mathcal{I}} \mathbb{P}\left[Y^{x, N} \in \mathcal{K}_{i}^{x}(M)\right]\right\} .
\end{aligned}
$$

Applying first Lemma 6.13 and then (111), we obtain

$$
\begin{aligned}
\limsup _{N \rightarrow \infty} \frac{1}{N} \log \mathbb{P}\left[Y^{x, N} \in \mathcal{K}_{i}^{x}(M)\right] & \leq-\inf _{\phi \in \mathcal{K}_{i}^{x}(M)} I^{\delta}\left(\phi, \theta_{i}\right) \\
& \leq-I^{\delta}\left(\phi_{i}\right)+\epsilon \\
& <-K+\epsilon
\end{aligned}
$$

as $I^{\delta}\left(\phi_{i}\right)>K$ (recall that $\left.i \in \mathcal{I}\right)$. The result now follows from the two last inequalities, Lemma 6.11 and the fact that $a \frac{M}{\log M}>K-\epsilon$.

\subsection{Main results}

Theorem 6.15. Assume that Assumption 2.3 is satisfied. For $F \subset D([0, T] ; A)$ closed and $x \in A$, we have

$$
\limsup _{y_{N} \in A^{N}, y_{N} \rightarrow x, N \rightarrow \infty} \frac{1}{N} \log \mathbb{P}\left[Z^{N, y_{N}} \in F\right] \leq-I_{x}(F) .
$$

Proof. We first let $I_{x}(F)=: K<\infty$ and $\epsilon>0$. By Lemma 4.23, there exits a $\delta^{\epsilon}>0$ such that for all $\delta \leq \delta^{\epsilon}$,

$$
y \in A,|x-y|<\delta \Rightarrow I_{y}(F) \geq I_{x}(F)-\epsilon=K-\epsilon .
$$

For $\delta \leq \delta^{\epsilon}$, we define

$$
\begin{aligned}
F^{\delta} & :=\{\phi \in F|| \phi(0)-x \mid \leq \delta\}, \\
S^{\delta} & :=\bigcup_{y \in A,|x-y| \leq \delta} \Phi_{y}(K-2 \epsilon) .
\end{aligned}
$$

$F^{\delta}$ is closed in $D\left([0, T] ; A ; \mathrm{d}_{D}\right)$ and $S^{\delta}$ is compact in $D\left([0, T] ; A ; \mathrm{d}_{D}\right)$ by Proposition 4.21, Furthermore, the two sets have no common elements. Hence, by the Hahn-Banach Theorem,

$$
\mathrm{d}\left(F^{\delta}, S^{\delta}\right)=: \eta^{\delta}>0
$$


Note that $\eta^{\delta}$ is increasing as $\delta$ is decreasing, since the sets $F^{\delta}$ and $S^{\delta}$ are decreasing. We now let $|y-x| \leq \delta$ and $\eta \leq \eta^{\delta}$. Let $Y^{N}$ be defined as in the paragraph preceding Lemma 6.1. We have

$$
\begin{aligned}
\mathbb{P}\left[Z^{N, y} \in F\right] & =\mathbb{P}\left[Z^{N, y} \in F^{\delta}\right] \\
& \leq \mathbb{P}\left[\mathrm{d}\left(Y^{N, y}, F^{\delta}\right)<\frac{\eta}{2}\right]+\mathbb{P}\left[\left\|Y^{N, y}-Z^{N, y}\right\| \geq \frac{\eta}{2}\right] .
\end{aligned}
$$

Let now $\phi(0)=y$ with $\mathrm{d}\left(\phi, F^{\delta}\right)<\frac{\eta}{2}$, hence from (113)

$$
\mathrm{d}\left(\phi, \Phi_{y}(K-2 \epsilon)\right) \geq \frac{\eta}{2}
$$

Let $\tilde{\delta}$ be such that Corollary 6.6 with $K$ replaced by $K-2 \epsilon$ and $\epsilon$ by $\frac{\eta}{4}$ holds with $\delta$ replaced by $2 \tilde{\delta}$. Hence (115) implies

$$
\mathrm{d}\left(\phi, \Phi_{y}^{2 \tilde{\delta}}\left(K-2 \epsilon-\frac{\eta}{4}\right)>\frac{\eta}{4} .\right.
$$

Indeed, if that is not the case, there exists a $\tilde{\phi} \in \Phi_{y}^{2 \tilde{\delta}}\left(K-2 \epsilon-\frac{\eta}{4}\right)$ with $\|\phi-\tilde{\phi}\| \leq \frac{\eta}{4}$. Then Corollary 6.6 implies that there exists $\bar{\phi} \in \Phi_{y}(K-2 \epsilon)$ with $\|\bar{\phi}-\tilde{\phi}\| \leq \frac{\eta}{4}$; consequently $\|\bar{\phi}-\phi\| \leq \frac{\eta}{2}$, which contradicts (116). We hence obtain by Lemma 6.14,

$$
\begin{aligned}
\limsup _{N \rightarrow \infty} \frac{1}{N} \log \mathbb{P}\left[\mathrm{d}\left(Y^{N, y}, F^{\delta}\right)<\frac{\eta}{2}\right] & \leq \limsup _{N \rightarrow \infty} \frac{1}{N} \log \mathbb{P}\left[\mathrm{d}\left(Y^{N, y}, \Phi_{y}^{2 \tilde{\Delta}}\left(K-2 \epsilon-\frac{\eta}{4}\right)>\frac{\eta}{4}\right]\right. \\
& \leq-\left(K-2 \epsilon-\frac{\eta}{2}\right)
\end{aligned}
$$

uniformly in $y \in A$ with $|y-x| \leq \delta$.

Furthermore, Lemma 6.1 implies

$$
\limsup _{N \rightarrow \infty} \frac{1}{N} \log \mathbb{P}\left[\left\|Y^{N, y}-Z^{N, y}\right\| \geq \frac{\eta}{2}\right]=-\infty
$$

uniformly in $y \in A$.

Combining Inequalities (114), (117) and (118), we obtain

$$
\limsup _{N \rightarrow \infty} \frac{1}{N} \log \mathbb{P}\left[Z^{N, y} \in F\right] \leq-\left(K-2 \epsilon-\frac{\eta}{2}\right)
$$

uniformly in $y \in A,|x-y| \leq \delta$. The result now follows as $\epsilon$ and $\eta$ can be chosen arbitrarily small.

The result in case $I_{x}(F)=\infty$ follows, since this implies that $I_{x}(F)>K$ for all $K>0$.

We will need the following stronger version. Recall the definition of $A^{N}$ at the start of section 2 ,

Theorem 6.16. Assume that Assumption 2.3 is satisfied. For $F \subset D([0, T] ; A)$ closed and any compact subset $K \subset A$, we have

$$
\limsup _{N \rightarrow \infty} \frac{1}{N} \log \sup _{x \in K \cap A^{N}} \mathbb{P}\left[Z^{N, x} \in F\right] \leq-\inf _{x \in K} I_{x}(F) .
$$


Proof. We use the same argument as in the proof of Corollary 5.6.15 in Dembo and Zeitouni [2009]. From Theorem 6.15, for any $x \in A$, any $\delta>0$, there exists $\epsilon_{x, \delta}>0$ and $N_{x, \delta} \geq 1$ such that whenever $N \geq N_{x, \delta}, y \in A_{N}$ with $|y-x|<\epsilon_{x, \delta}$,

$$
\frac{1}{N} \log \mathbb{P}\left[Z^{N, y} \in F\right] \leq-I_{x}^{\delta}(F),
$$

where $I_{x}^{\delta}(F)=\min \left[I_{x}(F)-\delta, \delta^{-1}\right]$. Consider now a compact set $K \subset A$. There exists a finite set $\left\{x_{i}, 1 \leq i \leq I\right\}$ such that $K \subset \cup_{i=1} B\left(x_{i}, \epsilon_{x_{i}}\right)$, where $B(x, \epsilon)=\{y ;|y-x|<\epsilon\}$. Consequently, for $N \geq \sup _{1 \leq i \leq I} N_{x_{i}, \delta}$, any $y \in A_{N} \cap K$,

$$
\frac{1}{N} \log \mathbb{P}\left[Z^{N, y} \in F\right] \leq-\min _{1 \leq i \leq I} I_{x_{i}}^{\delta}(F) \leq-\inf _{x \in K} I_{x}^{\delta}(F) .
$$

It remains to take the sup over $y \in K \cap A_{N}$ on the left, take the $\lim \sup$ as $N \rightarrow \infty$, and finally let $\delta$ tend to 0 to deduce the result.

\section{Time of exit from domain}

In this section we establish the results for the time of exit of the process from a domain; to this end, we follow the line of reasoning of Dembo and Zeitouni [2009] Section 5.7 and modify the arguments when necessary.

We let $O \subsetneq A$ be relatively open in $A$ (with $O=\tilde{O} \cap A$ for $\tilde{O} \subset \mathbb{R}^{d}$ open) and $x^{*} \in O$ be a stable equilibrium of (2). By a slight abuse of notation, we say that

$$
\widetilde{\partial O}:=\partial \tilde{O} \cap A
$$

is the boundary of $O$. For $y, z \in A$, we define the following functionals.

$$
\begin{aligned}
V(x, z, T) & :=\inf _{\phi \in D([0, T] ; A), \phi(0)=x, \phi(T)=z} I_{T, x}(\phi) \\
V(x, z) & :=\inf _{T>0} V(x, z) \\
\bar{V} & :=\inf _{z \in \widetilde{\partial O}} V\left(x^{*}, z\right) .
\end{aligned}
$$

In other words, $\bar{V}$ is the minimal energy required to leave the domain $O$ when starting from $x^{*}$.

Assumption 7.1. (D1) $x^{*}$ is the only stable equilibrium point of (2) in $O$ and the solution $Y^{x}$ of (2) with $x=Y^{x}(0) \in O$ satisfies

$$
Y^{x}(t) \in O \text { for all } t>0 \text { and } \lim _{t \rightarrow \infty} Y^{x}(t)=x^{*} .
$$

(D2) For a solution $Y^{x}$ of (2) with $x=Y^{x}(0) \in \widetilde{\partial O}$, we have

$$
\lim _{t \rightarrow \infty} Y^{x}(t)=x^{*} .
$$

(D3) $\bar{V}<\infty$. 
(D4) For all $\rho>0$ there exist constants $T(\rho), \epsilon(\rho)>0$ with $T(\rho), \epsilon(\rho) \downarrow 0$ as $\rho \downarrow 0$ such that for all $z \in \widetilde{\partial O} \cup\left\{x^{*}\right\}$ and all $x, y \in \overline{B(z, \rho)} \cap A$ there exists an

$$
\phi=\phi(\rho, x, y):[0, T(\rho)] \rightarrow A \quad \text { with } \phi(0)=x, \phi(T(\rho))=y \text { and } I_{T(\rho)}(\phi)<\epsilon(\rho) .
$$

(D5) For all $z \in \widetilde{\partial O}$ there exists an $\eta_{0}>0$ such that for all $\eta<\eta_{0}$ there exists a $\tilde{z}=\tilde{z}(\eta) \in A \backslash \bar{O}$ with $|z-\tilde{z}|>\eta$.

Let us shortly comment on Assumption 7.1. By (D1), $O$ is a subset of the domain of attraction of $x^{*}$. (D2) is violated by the applications we have in mind: we are interested in situations where $\widetilde{\partial O}$ is the characteristic boundary of $O$, i.e., the boundary separating two regions of attraction of equilibria of (2). In order to relax this assumption, we shall add an approximation argument in section 7.3. By (D3), it is possible to reach the boundary with finite energy. This assumption is always satisfied for the epidemiological models we consider. For $z=x^{*}$, (D4) is also always satisfied in our models as the rates $\beta_{j}$ are bounded from above and away from zero in small neighborhoods of $x^{*}$; hence, the function $\phi(x, y, \rho)$ can, e.g., be chosen to be linear with speed one (see, e.g., Shwartz and Weiss [1995] Lemma 5.22). (D5) allows us to consider a trajectory which crosses the boundary $\widetilde{\partial O}$, in such a way that all paths in a sufficiently small tube around that trajectory do exit $O$.

We are interested in the following quantity:

$$
\tau^{N, x}:=\tau^{N}:=\inf \left\{t>0 \mid Z^{N, x}(t) \notin O\right\},
$$

i.e., the first time that $Z^{N, x}$ exits $O$.

\subsection{Auxiliary results}

Assumption 7.1 (A4) gives the following analogue of Lemma 5.7.8 of Dembo and Zeitouni [2009].

Lemma 7.2. Assume that Assumption 7.1 holds. Then for any $\delta>0$, there exists an $\rho_{0}>0$ such that for all $\rho<\rho_{0}$,

$$
\sup _{z \in \widetilde{\partial O} \cup x^{*}, x, y \in \overline{B(z, \rho)}} \inf _{T \in[0,1]} V(x, y, T)<\delta .
$$

We can recover the analogue of Lemma 5.7.18 of Dembo and Zeitouni [2009] by using Lemma 7.2 .

Lemma 7.3. Assume that Assumptions 2.3 and 7.1 hold. Then, for any $\eta>0$ there exists a $\rho_{0}$ such that for all $\rho<\rho_{0}$ there exists a $T_{0}<\infty$ such that

$$
\liminf _{N \rightarrow \infty} \frac{1}{N} \log \underset{x \in \overline{B\left(x^{*}, \rho\right)}}{\inf } \mathbb{P}\left[\tau^{N, x} \leq T_{0}\right]>-(\bar{V}+\eta) .
$$

Proof. We follow the same line of reasoning as in the proof of Lemma 5.7.18 in Dembo and Zeitouni [2009]. Let $x \in \overline{B\left(x^{*}, \rho\right)}$. We use Lemma 7.2 for $\delta=\eta / 4$ (and we let $\rho$ be small enough for Lemma 7.2 to hold). We construct a continuous path $\psi^{x}$ with $\psi^{x}(0)=x, \psi^{x}\left(t_{x}\right)=x^{*}$ 
$\left(t_{x} \leq 1\right)$ and $I_{t_{x}, x}\left(\psi^{x}\right) \leq \eta / 4$. We then use Assumption 7.1 (D3). For $T_{1}<\infty$, we can construct a path $\phi \in C\left[0, T_{1}\right]$ such that $\phi(0)=x^{*}, \phi\left(T_{1}\right)=z \in \widetilde{\partial O}$ and $I_{T_{1}, 0}(\phi) \leq \bar{V}+\eta / 4$. Subsequently, we use Lemma 7.2 and obtain a path $\tilde{\psi}$ with $\tilde{\psi}(0)=z, \tilde{\psi}\left(s_{x}\right) \notin O(s \leq 1)$, $I_{s, z}(\tilde{\psi}) \leq \eta / 4$ and $\operatorname{dist}(\bar{z}, O)=: \Delta>0.10$ We finally let $\theta^{x}$ be the solution of the ODE (2) with $\theta^{x}(0)=\bar{z}$ on $\left[0,2-t_{x}-s\right]$, consequently $I_{2-t_{x}-s, \bar{z}}\left(\theta^{x}\right)=0$, see Lemma 4.1.

We concatenate the paths $\psi^{x}, \phi, \tilde{\psi}$ and $\theta^{x}$ and obtain the path $\phi^{x} \in C\left[0, T_{0}\right]\left(T_{0}=\right.$ $T_{1}+2$ independent of $\left.x\right)$ with $I_{T_{0}, x}\left(\phi^{x}\right) \leq \bar{V}+\eta / 2$.

Finally, we define

$$
\Psi:=\bigcup_{x \in \overline{B\left(x^{*}, \rho\right)}}\left\{\psi \in D\left(\left[0, T_{0}\right] ; A\right) \mid\left\|\psi-\phi^{x}\right\|<\Delta / 2\right\}
$$

hence $\Psi \subset D\left(\left[0, T_{0}\right] ; A\right)$ is open, $\left(\phi^{x}\right)_{x \in \overline{B\left(x^{*}, \rho\right)}} \subset \Psi$ and $\left\{Z^{N, x} \in \Psi\right\} \subset\left\{\tau^{N, x} \leq T_{0}\right\}$. We now use Theorem 5.11 .

$$
\begin{aligned}
\liminf _{N \rightarrow \infty} \frac{1}{N} \log \inf _{x \in \overline{B\left(x^{*}, \rho\right)}} \mathbb{P}\left[Z^{N, x} \in \Psi\right] & \geq-\sup _{x \in \overline{B\left(x^{*}, \rho\right)}} \inf _{\phi \in \Psi} I_{T_{0}, x}(\phi) \\
& \geq-\sup _{x \in \overline{B\left(x^{*}, \rho\right)}} I_{T_{0}, x}\left(\phi^{x}\right) \\
& >-(\bar{V}+\eta) .
\end{aligned}
$$
2009])

We also require the following result (analogue of Lemma 5.7.19 of Dembo and Zeitouni

Lemma 7.4. Assume that Assumption 7.1 holds. Let $\rho>0$ such that $\overline{B\left(x^{*}, \rho\right)} \subset O$ and

$$
\sigma_{\rho}^{N, x}:=\inf \left\{t>0 \mid Z_{t}^{N, x} \in \overline{B\left(x^{*}, \rho\right)} \text { or } Z_{t}^{N, x} \notin O\right\} .
$$

Then

$$
\lim _{t \rightarrow \infty} \limsup _{N \rightarrow \infty} \frac{1}{N} \log \sup _{x \in O} \mathbb{P}\left[\sigma_{\rho}^{N, x}>t\right]=-\infty .
$$

Proof. We adapt the proof of Dembo and Zeitouni [2009] Lemma 5.7.19 to our case.

Note first that for $x \in \overline{B\left(x^{*}, \rho\right)}, \sigma_{\rho}^{N, x}=0$; we hence assume from now on that $x \notin$ $\overline{B\left(x^{*}, \rho\right)}$. For $t>0$, we define the closed set $\Psi_{t} \subset D([0, t] ; A)$,

$$
\Psi_{t}:=\left\{\phi \in D([0, t] ; A) \mid \phi(s) \in \overline{O \backslash B\left(x^{*}, \rho\right)} \text { for all } s \in[0, t]\right\}
$$

hence for all $x, N$,

$$
\left\{\sigma_{\rho}^{N, x}>t\right\} \subset\left\{Z^{N, x} \in \Psi_{t}\right\} .
$$

By Theorem 6.16, this implies for all $t>0$,

$$
\limsup _{N \rightarrow \infty} \frac{1}{N} \log \sup _{x \in \overline{O \backslash B\left(x^{*}, \rho\right)}} \mathbb{P}\left[\sigma_{\rho}^{N, x}>t\right] \leq \limsup _{N \rightarrow \infty} \frac{1}{N} \log \sup _{x \in \overline{O \backslash B\left(x^{*}, \rho\right)}} \mathbb{P}\left[Z^{\epsilon, x} \in \Psi_{t}\right]
$$

\footnotetext{
${ }^{10}$ Note that the Assumption (D5) is required here.
} 


$$
\leq-\inf _{\phi \in \Psi_{t}} I_{t, \phi(0)}(\phi)
$$

It hence suffices to show that

$$
\lim _{t \rightarrow \infty} \inf _{\phi \in \Psi_{t}} I_{t, \phi(0)}(\phi)=\infty
$$

To this end, consider $x \in \overline{O \backslash B\left(x^{*}, \rho\right)}$ and recall that $Y^{x}$ is the solution of (2) (on

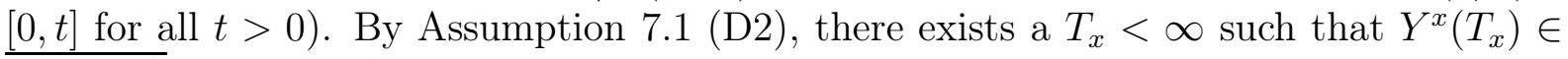
$\overline{B\left(x^{*}, 3 \rho\right)}$. We have (here $B$ denotes the Lipschitz constant of $b$ ),

$$
\left|\phi^{x}(t)-\phi^{y}(t)\right| \leq|x-y|+\int_{0}^{t}\left|b\left(\phi^{x}(s)\right)-b\left(\phi^{y}(s)\right)\right| d s \leq+|x-y|+\int_{0}^{t} B\left|\phi^{x}(s)-\phi^{y}(s)\right| d s
$$

and therefore by Gronwall's inequality $\left|Y^{x}\left(T_{x}\right)-Y^{y}\left(T_{x}\right)\right| \leq|x-y| e^{T_{x} B}$; consequently, there exists a neighborhood $W_{x}$ of $x$ such that for all $y \in W_{x}, Y^{y}\left(T_{x}\right) \in \overline{B\left(x^{*}, 3 \rho\right)}$. By the compactness of $\overline{O \backslash B\left(x^{*}, \rho\right)}$, there exists a finite open subcover $\cup_{i=1}^{k} W_{x_{i}} \supseteq \overline{O \backslash B\left(x^{*}, \rho\right)}$; for $T:=\max _{i=1, \ldots, k} T_{x_{i}}$ and $y \in \overline{O \backslash B\left(x^{*}, \rho\right)}$ this implies that $Y^{y}(s) \in \overline{B\left(x^{*}, 2 / 3 \rho\right)}$ for some $s \leq T$.

Assume now that (119) is false. Then there exits an $M<\infty$ such that for all $n \in \mathbb{N}$ there exists an $\phi_{n} \in \Psi_{n T}$ with $I_{n T}\left(\phi_{n}\right) \leq M$. The function $\phi_{n}$ is concatenated by functions $\phi_{n, k} \in \Psi_{T}$ and we obtain

$$
M \geq I_{n T}\left(\phi_{n}\right)=\sum_{k=1}^{n} I_{T}\left(\phi_{n, k}\right) \geq n \min _{k=1, \ldots, n} I_{T}\left(\phi_{n, k}\right)
$$

Hence there exists a sequence $\left(\psi_{k}\right)_{k} \subset \Psi_{T}$ with $\lim _{k \rightarrow \infty} I_{T}\left(\psi_{k}\right)=0$. Note now that the set

$$
\phi(t):=\left\{\phi \in C[0, T] \mid I_{T, \phi(0)}(\phi) \leq 1, \phi(s) \in \overline{O \backslash B\left(x^{*}, \rho\right)} \text { for all } s \in[0, T]\right\} \subset \Psi_{T}
$$

is compact (as a subset of $\left.\left(C[0, T],\|\cdot\|_{\infty}\right)\right)$; hence there exists a subsequence $\left(\psi_{k_{l}}\right)_{l}$ of $\left(\psi_{k}\right)_{k}$ such that $\lim _{l \rightarrow \infty} \psi_{k_{l}}=: \psi^{*} \in \phi(t)$ in $\left(C[0, T],\|\cdot\|_{\infty}\right)$. By the lower semi-continuity of $I_{T}$ (cf. Lemma 4.20) this implies

$$
0=\liminf _{l \rightarrow \infty} I_{T}\left(\psi_{n_{l}}\right) \geq I_{T}\left(\psi^{*}\right),
$$

which in turn implies that $\psi^{*}$ solves (2) for $x=\psi^{*}(0)$. But then, $\psi^{*}(s) \in \overline{B\left(x^{*}, 2 / 3 \rho\right)}$ for some $s \leq T$, a contradiction to $\psi^{*} \in \Psi_{T}$.

The following lemma is the analogue of Dembo and Zeitouni [2009] Lemma 5.7.21.

Lemma 7.5. Assume that Assumptions 2.3 and 7.1 hold. Let $C \subset A \backslash O$ be closed. Then

$$
\lim _{\rho \rightarrow 0} \limsup _{N \rightarrow \infty} \frac{1}{N} \log \underset{x \in \overline{B\left(x^{*}, 3 \rho\right) \backslash B\left(x^{*}, 2 \rho\right)}}{\sup } \mathbb{P}\left[Z_{\sigma_{\rho}}^{N, x} \in C\right] \leq-\inf _{z \in C} V\left(x^{*}, z\right) .
$$


Proof. We adapt the proof of Dembo and Zeitouni [2009] Lemma 5.7.21 to our situation. We can assume without loss of generality that $\inf _{z \in C} V\left(x^{*}, z\right)>0$ (else the assertion is trivial). For $\inf _{z \in C} V\left(x^{*}, z\right)>\delta>0$, we define

$$
V_{C}^{\delta}:=\left(\inf _{z \in C} V\left(x^{*}, z\right)-\delta\right) \wedge 1 / \delta>0 .
$$

By Lemma 7.2, there exists a $\rho_{0}=\rho_{0}(\delta)>0$ such that for all $0<\rho<\rho_{0}$,

$$
\sup _{y \in \overline{B\left(x^{*}, 3 \rho\right) \backslash B\left(x^{*}, 2 \rho\right)}} V\left(x^{*}, y\right)<\delta
$$

hence

$$
\inf _{y \in \overline{B\left(x^{*}, 3 \rho\right) \backslash B\left(x^{*}, 2 \rho\right)},}=C(y, z) \geq \inf _{z \in C} V\left(x^{*}, z\right)-\sup _{y \in \overline{B\left(x^{*}, 3 \rho\right) \backslash B\left(x^{*}, 2 \rho\right)}} V\left(x^{*}, y\right)>V_{C}^{\delta} .
$$

For $T>0$, we define the closed set $\Phi^{T} \subset D([0, T] ; A)$ by

$$
\Phi^{T}:=\Phi:=\{\phi \in D([0, T] ; A) \mid \phi(t) \in C \text { for some } t \in[0, T]\} .
$$

We then have for $y \in \overline{B\left(x^{*}, 3 \rho\right) \backslash B\left(x^{*}, 2 \rho\right)}$,

$$
\mathbb{P}\left[Z_{\sigma_{\rho}}^{N, y} \in C\right] \leq \mathbb{P}\left[\sigma_{\rho}^{N, y}>T\right]+\mathbb{P}\left[Z^{N, y} \in \Phi^{T}\right] .
$$

In the following, we bound the two parts in Inequality (121) from above.

For the second part, we note first that (cf. Inequality (120))

$$
\inf _{y \in \overline{B\left(x^{*}, 3 \rho\right) \backslash B\left(x^{*}, 2 \rho\right)}, \phi \in \Phi^{T}} I_{T, y}(\phi) \geq \inf _{y \in \overline{B\left(x^{*}, 3 \rho\right) \backslash B\left(x^{*}, 2 \rho\right)}, z \in C} V(y, z)>V_{C}^{\delta} ;
$$

hence, we obtain by Theorem 6.16

$$
\begin{aligned}
\limsup _{N \rightarrow \infty} \frac{1}{N} \log \sup _{y \in \overline{B\left(x^{*}, 3 \rho\right) \backslash B\left(x^{*}, 2 \rho\right)}} \mathbb{P}\left[Z^{N, y} \in \Phi^{T}\right] & \leq-\inf _{y \in \overline{B\left(x^{*}, 3 \rho\right) \backslash B\left(x^{*}, 2 \rho\right)}, \phi \in \Phi^{T}} I_{T, y}(\phi) \\
& <-V_{C}^{\delta} .
\end{aligned}
$$

For the first part in Inequality (121), we use Lemma 7.4; There exists a $0<T_{0}<\infty$ such that for all $T \geq T_{0}$

$$
\limsup _{N \rightarrow \infty} \frac{1}{N} \log \sup _{y \in \overline{B\left(x^{*}, 3 \rho\right) \backslash B\left(x^{*}, 2 \rho\right)}} \mathbb{P}\left[\sigma^{N, y}>T\right]<-V_{C}^{\delta} .
$$

We let $T \geq T_{0}$ and $\rho<\rho_{0}$ and combine Inequalities (121), (122) and (123). Hence there exists an $N_{0}>0$ such that for all $N>N_{0}$,

$$
\begin{aligned}
& \frac{1}{N} \log \underset{y \in \overline{B\left(x^{*}, 3 \rho\right) \backslash B\left(x^{*}, 2 \rho\right)}}{ } \mathbb{P}\left[Z_{\sigma_{\rho}}^{N, y} \in C\right] \\
& \quad \leq \frac{1}{N} \log \left(\sup _{y \in \overline{B\left(x^{*}, 3 \rho\right) \backslash B\left(x^{*}, 2 \rho\right)}} \mathbb{P}\left[\sigma_{\rho}^{N, y}>T\right]+\sup _{y \in \overline{B\left(x^{*}, 3 \rho\right) \backslash B\left(x^{*}, 2 \rho\right)}} \mathbb{P}\left[Z^{N, y} \in \Phi^{T}\right]\right) \\
& \quad<\frac{1}{N} \log \left(2 e^{-N V_{C}^{\delta}}\right)=\frac{1}{N} \log 2-V_{C}^{\delta} ;
\end{aligned}
$$

and

$$
\limsup _{N \rightarrow \infty} \frac{1}{N} \log \sup _{y \in \overline{B\left(x^{*}, 3 \rho\right) \backslash B\left(x^{*}, 2 \rho\right)}} \mathbb{P}\left[Z_{\sigma_{\rho}}^{N, x} \in C\right] \leq-V_{C}^{\delta} .
$$

Taking the limit $\delta \rightarrow 0$ finishes the proof. 
The next lemma is the analogue of Lemma 5.7.22 of Dembo and Zeitouni [2009].

Lemma 7.6. Assume that Assumption 7.1 holds. Then, for all $\rho>0$ such that $\overline{B\left(x^{*}, \rho\right)} \subset$ $O$ and for all $x \in O$,

$$
\lim _{N \rightarrow \infty} \mathbb{P}\left[Z_{\sigma_{\rho}}^{N, x} \in \overline{B\left(x^{*}, \rho\right)}\right]=1 .
$$

Proof. Let $x \in O \backslash \overline{B\left(x^{*}, \rho\right)}$ (the case $x \in \overline{B\left(x^{*}, \rho\right)}$ is clear). Let furthermore $T:=\inf \{t \geq$ $\left.0 \mid \phi(t) \in B\left(x^{*}, \rho / 2\right)\right\}$. Since $Y^{x}$ is continuous and never reaches $\widetilde{\partial O}$ (Assumption 7.1(D1)), we have $\inf _{t \geq 0} \operatorname{dist}\left(Y^{x}(t), \widetilde{\partial O}\right)=: \Delta>0$. Hence we have the following implication:

$$
\sup _{t \in[0, T]}\left|Z_{t}^{N, x}-Y^{x}(t)\right| \leq \frac{\Delta}{2} \Rightarrow Z_{\sigma_{\rho}}^{N, x} \in \overline{B\left(x^{*}, \rho\right)} .
$$

In other words,

$$
\mathbb{P}\left[Z_{\sigma_{\rho}}^{N, x} \notin \overline{B\left(x^{*}, \rho\right)}\right] \leq \mathbb{P}\left[\sup _{t \in[0, T]}\left|Z_{t}^{N, x}-Y^{x}(t)\right|>\frac{\Delta}{2}\right]
$$

The right hand side of Inequality (124) converges to zero as $N \rightarrow \infty$ by Theorem 3.1

The next lemma is the analogue of Dembo and Zeitouni [2009] Lemma 5.7.23.

Lemma 7.7. Assume that Assumption 7.1 holds. Then, for all $\rho, c>0$, there exists a constant $T=T(c, \rho)<\infty$ such that

$$
\limsup _{N \rightarrow \infty} \frac{1}{N} \log \sup _{x \in O} \mathbb{P}\left[\sup _{t \in[0, T]}\left|Z_{t}^{N, x}-x\right| \geq \rho\right]<-c .
$$

Proof. Let $\rho, c>0$ be fixed. For $T, N>0$ and $x \in O$ we have

$$
\begin{aligned}
\mathbb{P}\left[\sup _{t \in[0, T]}\left|Z_{t}^{N, x}-x\right| \geq \rho\right] & =\mathbb{P}\left[\sup _{t \in[0, T]} \frac{1}{N}\left|\sum_{j} h_{j} P_{j}\left(N \int_{0}^{t} \beta_{j}\left(Z_{s}^{N, x}\right) d s\right)\right| \geq \rho\right] \\
& \leq \mathbb{P}\left[\sum_{j} P_{j}(N \bar{\beta} T) \geq N \rho \bar{h}^{-1}\right] \\
& \leq k \mathbb{P}\left[P(N \bar{\beta} T) \geq N \rho \bar{h}^{-1} k^{-1}\right]
\end{aligned}
$$

for a standard Poisson process $P$. We now let, with $c_{1}(T)=\bar{\beta} T$ and $c_{2}=\rho \bar{h}^{-1} k^{-1}$,

$$
T<T_{0}:=\frac{e^{-1} c_{2}}{2 \bar{\beta}} \wedge \frac{e^{-c / c_{2}-1} c_{2}}{\bar{\beta}} \text { and } \quad N>N_{0}:=1 / c_{2} \wedge \frac{\log 2 k}{c_{1}(T)} .
$$

We then obtain (note that $N c_{2}>1$ and $\frac{e}{c_{2}} c_{1}(T)<1 / 2$ by $(\underline{126})$ )

$$
\begin{aligned}
k \mathbb{P}\left[P\left(N c_{1}(T)\right) \geq N c_{2}\right] & =k e^{-N c_{1}(T)} \sum_{m \geq N c_{2}} \frac{N^{m} c_{1}(T)^{m}}{m !} \\
& <k e^{-N c_{1}(T)} \sum_{m \geq N c_{2}} \frac{(e N)^{m} c_{1}(T)^{m}}{m^{m} \sqrt{2 \pi m}}
\end{aligned}
$$




$$
\begin{aligned}
& \leq \frac{1}{2} \sum_{m \geq N c_{2}} \frac{(e N)^{m} c_{1}(T)^{m}}{\left(N c_{2}\right)^{m}} \\
& \leq \frac{1}{2} \frac{\left(\frac{e}{c_{2}} c_{1}(T)\right)^{N c_{2}}}{1-\frac{e}{c_{2}} c_{1}(T)} \\
& \leq\left(\frac{e}{c_{2}} c_{1}(T)\right)^{N c_{2}} ;
\end{aligned}
$$

here we applied Stirling's formula, $m !>\sqrt{2 \pi m}(m / e)^{m}$, in Inequality (127). Finally, we have

$$
\left(\frac{e}{c_{2}} c_{1}(T)\right)^{N c_{2}}=\left(\left(\frac{e}{c_{2}} c_{1}(T)\right)^{-c_{2}}\right)^{-N}<\left(e^{c}\right)^{-N}=e^{-N c}
$$

by (126). The assertion now follows by combining the Inequalities (125), (128) and (129).

\subsection{Main results}

We can now deduce the analogue of Dembo and Zeitouni [2009] Theorem 5.7.11 (a). the proof of Dembo and Zeitouni [2009] carries over.

Theorem 7.8. Assume that Assumption 7.1 holds. Then, for all $x \in O \cap A N$ and $\delta>0$,

$$
\lim _{N \rightarrow \infty} \mathbb{P}\left[e^{(\bar{V}-\delta) N}<\tau^{N, x}<e^{(\bar{V}+\delta) N}\right]=1 .
$$

Moreover, for all $x \in O$, as $N \rightarrow \infty$,

$$
\frac{1}{N} \log \mathbb{E}\left(\tau^{N, x}\right) \rightarrow \bar{V}
$$

Proof. Upper bound of exit time:

We fix $\delta>0$ and apply Lemma 7.3 with $\eta:=\delta / 4$. Hence, for $\rho<\rho_{0}$ there exists a $T_{0}<\infty$ and an $N_{0}>0$ such that for $N>N_{0}$,

$$
\inf _{x \in \overline{B\left(x^{*}, \rho\right)}} \mathbb{P}\left[\tau^{N, x} \leq T_{0}\right]>e^{-N(\bar{V}+\eta)} .
$$

Furthermore, by Lemma 7.4 there exists a $T_{1}<\infty$ and $N_{1}>0$ such that for all $N>N_{1}$,

$$
\inf _{x \in O} \mathbb{P}\left[\sigma_{\rho}^{N, x} \leq T_{1}\right]>1-e^{-2 N \eta}
$$

For $T:=T_{0}+T_{1}$ and $N>N_{0} \vee N_{1} \vee 1 / \eta$, we hence obtain

$$
\begin{aligned}
& q^{N}:=q:=\inf _{x \in O} \mathbb{P}\left[\tau^{N, x} \leq T\right] \\
& \geq \inf _{x \in O} \mathbb{P}\left[\sigma_{\rho}^{N, x} \leq T_{1}\right] \inf _{y \in \overline{B\left(x^{*}, \rho\right)}} \mathbb{P}\left[\tau^{N, y} \leq T_{0}\right] \\
& >\left(1-e^{-2 N \eta}\right) e^{-N(\bar{V}+\eta)} \\
& \geq e^{-N(\bar{V}+2 \eta)} \text {. }
\end{aligned}
$$


This yields for $k \in \mathbb{N}$

$$
\begin{aligned}
\mathbb{P}\left[\tau^{N, x}>(k+1) T\right] & =\left(1-\mathbb{P}\left[\tau^{N, x} \leq(k+1) T \mid \tau^{N, x}>k T\right]\right) \mathbb{P}\left[\tau^{N, x}>k T\right] \\
& \leq(1-q) \mathbb{P}\left[\tau^{N, x}>k T\right]
\end{aligned}
$$

and hence inductively

$$
\sup _{x \in O} \mathbb{P}\left[\tau^{N, x}>k T\right] \leq(1-q)^{k} .
$$

This implies, exploiting (130) for the last inequality

$$
\sup _{x \in O} \mathbb{E}\left[\tau^{N, x}\right] \leq T\left(1+\sum_{k=1}^{\infty} \sup _{x \in O} \mathbb{P}\left[\tau^{N, x}>k T\right]\right) \leq T \sum_{k=0}^{\infty}(1-q)^{k}=\frac{T}{q} \leq T e^{N(\bar{V}+2 \eta)}
$$

by Chebychev's Inequality we obtain

$$
\mathbb{P}\left[\tau^{N, x} \geq e^{N(\bar{V}+\delta)}\right] \leq e^{-N(\bar{V}+\delta)} \mathbb{E}\left[\tau^{N, x}\right] \leq T e^{-\delta N / 2}
$$

which approaches zero as $N \rightarrow \infty$ as required.

Lower bound of exit time:

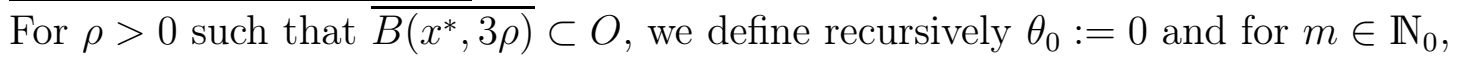

$$
\begin{aligned}
\tau_{m}^{x}:=\tau_{m} & :=\inf \left\{t \geq \theta_{m}^{x} \mid Z_{t}^{N, x} \in \overline{B\left(x^{*}, \rho\right)} \text { or } Z_{t}^{N, x} \notin O\right\}, \\
\theta_{m+1}^{x}:=\theta_{m+1} & :=\inf \left\{t \geq \tau_{m}^{x} \mid Z_{t}^{N, x} \in \overline{B\left(x^{*}, 3 \rho\right) \backslash B\left(x^{*}, 2 \rho\right)}\right\},
\end{aligned}
$$

with the convention $\theta_{m+1}:=\infty$ if $Z_{\tau_{m}}^{N} \notin O$. Note that we have $\tau^{N, x}=\tau_{m}^{x}$ for some $m \in \mathbb{N}_{0}$.

For fixed $T_{0}>0$ and $k \in \mathbb{N}$ we have the following implication: If for all $m=0, \ldots, k$, $\tau_{m} \neq \tau^{N}$ and for all $m=1 \ldots, k, \tau_{m}-\tau_{m-1}>T_{0}$, then

$$
\tau^{N}>\tau_{k}=\sum_{m=1}^{k}\left(\tau_{m}-\tau_{m-1}\right)>k T_{0}
$$

In particular, we have for $k:=\left\lfloor T_{0}^{-1} e^{N(\bar{V}-\delta)}\right\rfloor+1$ (note that $\theta_{m}-\tau_{m-1} \leq \tau_{m}-\tau_{m-1}$ ),

$$
\begin{aligned}
\mathbb{P}\left[\tau^{N, x} \leq e^{N(\bar{V}-\delta)}\right] & \leq \mathbb{P}\left[\tau^{N, x} \leq k T_{0}\right] \\
& \leq \sum_{m=0}^{k} \mathbb{P}\left[\tau^{N, x}=\tau_{m}^{x}\right]+\sum_{m=1}^{k} \mathbb{P}\left[\theta_{m}^{x}-\tau_{m-1}^{x} \leq T_{0}\right] \\
& =\mathbb{P}\left[\tau^{N, x}=\tau_{0}^{x}\right]+\sum_{m=1}^{k} \mathbb{P}\left[\tau^{N, x}=\tau_{m}^{x}\right]+\sum_{m=1}^{k} \mathbb{P}\left[\theta_{m}^{x}-\tau_{m-1}^{x} \leq T_{0}\right]
\end{aligned}
$$

In the following, we bound the three parts in (132) from above. To this end, we assume $\bar{V}>0$ for now. The simpler case $\bar{V}=0$ is treated below.

For the first part, we have

$$
\mathbb{P}\left[\tau^{N, x}=\tau_{0}^{x}\right]=\mathbb{P}\left[Z_{\sigma_{\rho}}^{N, x} \notin O\right] .
$$


For the second part, we use the fact that $Z^{N, x}$ is a strong Markov process and that the $\tau_{m}$ 's are stopping times. We obtain for $m \geq 1$ and $x \in O$,

$$
\mathbb{P}\left[\tau^{N, x}=\tau_{m}^{x}\right] \leq \sup _{y \in \overline{B\left(x^{*}, 3 \rho\right) \backslash B\left(x^{*}, 2 \rho\right)}} \mathbb{P}\left[Z_{\sigma_{\rho}}^{N, y} \notin O\right]
$$

Similarly, we obtain for the third part for $m \geq 1$ and $x \in O$,

$$
\mathbb{P}\left[\theta_{m}^{x}-\tau_{m-1}^{x} \leq T_{0}\right] \leq \sup _{y \in O} \mathbb{P}\left[\sup _{t \in\left[0, T_{0}\right]}\left|Z_{t}^{N, y}-y\right| \geq \rho\right]
$$

The upper bounds in (134) and (135) can now be bounded by using the Lemma 7.5 and 7.7, respectively. We fix $\delta>0$. By Lemma 7.5 (for $C=A \backslash O$ ), there exists a $\rho=\rho(\delta)>0$ and an $N_{1}=N_{1}(\rho, \delta)>0$ such that for all $N>N_{1}$,

$$
\sup _{y \in \overline{B\left(x^{*}, 3 \rho\right) \backslash B\left(x^{*}, 2 \rho\right)}} \mathbb{P}\left[Z_{\sigma_{\rho}}^{N, y} \notin O\right] \leq \exp (-N(\bar{V}-\delta / 2)) .
$$

By Lemma 7.7 (for $\rho=\rho(\delta)$ as above and $c=\bar{V}$ ), there exists a constant $T_{0}=T(\rho, \bar{V})<$ $\infty$ and an $N_{2}=N_{2}(\rho, \delta)>0$ such that for all $N>N_{2}$,

$$
\sup _{y \in O} \mathbb{P}\left[\sup _{t \in\left[0, T_{0}\right]}\left|Z_{t}^{N, y}-y\right| \geq \rho\right] \leq \exp (-N(\bar{V}-\delta / 2)) .
$$

We now let $N>N_{1} \vee N_{2}$ (and large enough for $T_{0}^{-1} \exp (N(\bar{V}-\delta))>1$ for the specific $T_{0}$ above). Then by Inequality (132),

$$
\begin{gathered}
\mathbb{P}\left[\tau^{N, x} \leq e^{N(\bar{V}-\delta)}\right] \stackrel{\text { (133), (134), (135) }}{\leq} \mathbb{P}\left[Z_{\sigma_{\rho}}^{N, x} \notin O\right]+k \sup _{y \in \overline{B\left(x^{*}, 3 \rho\right) \backslash B\left(x^{*}, 2 \rho\right)}} \mathbb{P}\left[Z_{\sigma_{\rho}}^{N, y} \notin O\right] \\
+k \sup _{y \in O} \mathbb{P}\left[\sup _{t \in\left[0, T_{0}\right]}\left|Z_{t}^{N, y}-y\right| \geq \rho\right] \\
\stackrel{[136),(137)}{\leq} \mathbb{P}\left[Z_{\sigma_{\rho}}^{N, x} \notin O\right]+4 T_{0}^{-1} \exp (-N \delta / 2) .
\end{gathered}
$$

The right-hand side of Inequality (138) tends to zero as $\epsilon \rightarrow 0$ by Lemma 7.6, finishing the proof for $\bar{V}>0$.

Finally, let us assume that $\bar{V}=0$ and that the assertion is false for a given $x \in O$. Then there exists a $\mu_{0} \in(0,1 / 2)$ and a $\delta_{0}>0$ such that for all $\bar{N}>0$ there exists an $N>\bar{N}$ with

$$
\mu_{0} \leq \mathbb{P}\left[\tau^{N, x} \leq e^{-N \delta_{0}}\right]
$$

We fix $\rho>0$ such that $\overline{B\left(x^{*}, 2 \rho\right)} \subset O$. Using the strong Markov property of $Z$ and the fact that $\sigma_{\rho}$ is a stopping time again, we have that for all $\bar{N}>0$ there exists an $N>\bar{N}$ with

$$
\begin{aligned}
\mu_{0} & \leq \mathbb{P}\left[\tau^{N, x} \leq e^{-N \delta_{0}}\right] \\
& \leq \mathbb{P}\left[Z_{\sigma_{\rho}}^{N, x} \notin \overline{B\left(x^{*}, \rho\right)}\right]+\sup _{y \in O} \mathbb{P}\left[\sup _{t \in\left[0, e^{-N \delta_{0}}\right]}\left|Z_{t}^{N, y}-y\right| \geq \rho\right] .
\end{aligned}
$$

By Lemma 7.6, there exists an $N_{0}$ such that for all $N>N_{0}$,

$$
\mathbb{P}\left[Z_{\sigma_{\rho}}^{N, x} \notin \overline{B\left(x^{*}, \rho\right)}\right] \leq \frac{\mu_{0}}{2} .
$$


We now set $c:=-2 \epsilon_{0} \log \frac{\mu_{0}}{2}$. Then by Lemma 7.7, there exists a $T=T(c, \rho)>0$ and an $N_{1}>N_{0}$ such that for all $N>N_{1}$,

$$
e^{-N \delta_{0}}<T
$$

and

$$
\sup _{y \in O} \mathbb{P}\left[\sup _{t \in[0, T]}\left|Z_{t}^{N, y}-y\right| \geq \rho\right] \leq e^{-N c / 2}<\frac{\mu_{0}}{2} .
$$

Combining Inequalities (140), (141) and (142) yields a contradiction to Inequality (139), finishing the proof.

Expected exit time:

We have shown in particular that $\mathbb{P}\left(\tau^{N, x}>e^{(\bar{V}-\delta) N}\right) \rightarrow 1$ as $N \rightarrow \infty$. Consequently, from Chebycheff,

$$
\begin{aligned}
\mathbb{E}\left(\tau^{N, x}\right) & \geq e^{(\bar{V}-\delta) N} \mathbb{P}\left(\tau^{N, x}>e^{(\bar{V}-\delta) N}\right), \\
\liminf _{N \rightarrow \infty} \frac{1}{N} \log \mathbb{E}\left(\tau^{N, x}\right) & \geq \bar{V}-\delta
\end{aligned}
$$

for all $\delta>0$. This together with (131) implies the second statement of the Theorem.

\subsection{The case of a characteristic boundary}

Since we are mainly interested in studying the time of exit form the basin of attraction of one local equilibrium to that of another, we need to consider situations which do not satisfy the above assumptions. More precisely, we want to suppress the Assumptions (D2), and keep Assumptions (D1), (D3), (D4) and (D5). We assume that there exists a collection of open sets $\left\{O_{\rho}, \rho>0\right\}$ which is such that

- $\bar{O}_{\rho} \subset O$ for any $\rho>0$.

- $d\left(O_{\rho}, \tilde{\partial O}\right) \rightarrow 0$, as $\rho \rightarrow 0$.

- $O_{\rho}$ satisfies Assumptions (D1), (D2), (D3), (D4) and (D5) for any $\rho>0$.

We can now establish

Corollary 7.9. Let then $O$ be a domain satisfying Assumptions (D1), (D3), (D4) and (D5), such that there exists a sequence $\left\{O_{\rho}, \rho>0\right\}$ satisfying the three above conditions. Then the conclusion of Theorem 7.8 is still true.

Proof. If we define $\bar{V}_{\rho}$ as $\bar{V}$, but with $O$ replaced by $O_{\rho}$, it follows from Lemma 7.2 that $\bar{V}_{\rho} \rightarrow \bar{V}$ as $\rho \rightarrow 0$. By an obvious monotonicity property and the continuity of the quasi-potential, the lower bound

$$
\lim _{N \rightarrow \infty} \mathbb{P}\left[\tau^{N, x}>e^{(\bar{V}-\delta) N}\right]=1
$$

follows immediately from Theorem 7.8 . The proof of the upper bound is done as in the proof of Theorem 7.8, once (130) is established. Let us now explain how this is done. Let 
$\tau_{\rho}^{N, x}$ denote the time of exit from $O_{\rho}$. The same argument used to establish (130) above leads to the statement that for any $\rho, \eta>0$, there exists $N_{\rho, \eta}$ such that for all $N \geq N_{\rho, \eta}$,

$$
\inf _{x \in O} \mathbb{P}\left[\tau^{N, x} \leq T\right] \geq e^{-N(\bar{V}+\eta)} .
$$

Now utilizing (D4), (D5) and the compactness of $\bar{O} \backslash O_{\rho}$, it is not hard to deduce from Theorem 5.11 that for $\rho>0$ small enough,

$$
\liminf _{N \rightarrow \infty} \log \inf _{x \in\left(\bar{O} \backslash O_{\rho}\right) \cap A^{N}} \mathbb{P}_{x}\left(\tau^{N, x} \leq 1\right)>-\eta .
$$

The wished result follows now from the last two lower bounds and the strong Markov property.

Finally the result for $\mathbb{E}\left(\tau^{N, x}\right)$ now follows from the first part of the result, exactly as in the proof of Theorem 7.8 ,

\section{Example: the SIRS model}

We finally show that Theorem 7.8 applies to the SIRS model from Example 1.1. Assumptions (A) and (B) have already been verified in Section 2. For (C), we note that major problems only occur for the balls centered at the "corner points" of the set $A$. Only for a corner point $x$ (with corresponding vector $v$ ) do we have $v \notin \mathcal{C}_{x}$ (recall that we define $\mathcal{C}_{x}$ corresponding to the modified rates $\beta^{\delta}$ ). For simplicity of exposition, we concentrate on the ball $B$ centered at $x=(1,0)^{\top}$. The same argument applies to the balls centered at the other corners and in a simpler form to all other balls. For the balls $B_{i}$ not centered at the corners, the vectors $v_{i}$ can be represented by $\mu^{i}$ s for which $\mu_{j}^{i}>0$ implies that $\beta_{j}^{\delta}(z)>\lambda>0$ (for an appropriate constant $\lambda$ which can be chosen independently of $i$ ) for all $z \in B_{i}$. This simplifies the discussion below significantly. In particular, Assumption (C) is satisfied due to Theorem 3.1. We first note that for all $x \in A, y \in \mathbb{R}^{d}$, $L(x, y)=\tilde{L}(x, y)$, cf. Theorem 4.9 below. As before, we define the vector

$$
v=(-1 / 2,1 / 4)^{\top} \quad \text { and } \quad \mu_{1}=0, \quad \mu_{2}=\frac{1}{2}, \quad \mu_{3}=\frac{1}{4},
$$

in particular $\mu \in \tilde{V}_{v}$ (but $V_{x, v}=\emptyset$ !). In order to simplify the notation, we do not normalize $v$. We let $\eta<\eta_{0}:=1 / 2$ and note that for $t \in[0, \eta]$,

$$
\beta_{2}\left(\phi^{x}(t)\right)=\gamma\left(1-\frac{t}{2}\right) \geq \frac{3}{4} \gamma, \quad \beta_{3}\left(\phi^{x}(t)\right)=\frac{\nu}{4} t .
$$

Let us prove that Assumption (C) is satisfied. Let $X^{N}$ be a Poisson random variable with mean $\mu N$. We note that by Theorem 3.1 for $\xi>1$,

$$
\mathbb{P}\left[X^{N}>\xi N \mu\right] \leq \bar{C}_{1} \exp \left(-N \bar{C}_{2}(\xi)\right), \quad \mathbb{P}\left[X^{N}<\xi^{-1} N \mu\right] \leq \tilde{C}_{1} \exp \left(-N \tilde{C}_{2}(\xi)\right)
$$

for appropriate constants $\bar{C}_{1}, \tilde{C}_{1}, \bar{C}_{2}, \tilde{C}_{2}$ with $\bar{C}_{2}(\xi)=O\left((\xi-1)^{2}\right)$ as $\xi \downarrow 1$ and $\tilde{C}_{2}(\xi)=$ $O\left(\left(1-\xi^{-1}\right)^{2}\right)$ as $\xi \downarrow 1$. The first bound is obtained by applying Theorem 3.1 to $d=k=$ $h_{1}=1, \beta_{1} \equiv \mu$ and $x=0$. We get

$$
\mathbb{P}\left[X^{N}>\xi N \mu\right]=\mathbb{P}\left[\frac{1}{N} X^{N}>\xi \mu\right]
$$




$$
\begin{aligned}
& =\mathbb{P}\left[\frac{1}{N} X^{N}-\mu>(\xi-1) \mu\right] \\
& \leq \mathbb{P}\left[\left|\frac{1}{N} X^{N}-\mu\right|>(\xi-1) \mu\right] \\
& =\mathbb{P}\left[\left|Z^{N, 0}(1)-\mu\right|>(\xi-1) \mu\right] \\
& \leq \mathbb{P}\left[\sup _{t \in[0,1]}\left|Z^{N, 0}(t)-\mu t\right|>(\xi-1) \mu\right] .
\end{aligned}
$$

Let us define the process $\hat{Z}^{N, x}$ as the solution of (3i) with constant rates $\mu_{j}$. For $\epsilon>0$ small enough, we define

$X_{2}^{N, \epsilon}:=$ \#jumps of type $h_{2}$ of $\tilde{Z}^{N, x}$ in $[0, \epsilon], \quad X_{3}^{N, \epsilon}:=$ \#jumps of type $h_{3}$ of $\tilde{Z}^{N, x}$ in $[0, \epsilon]$

and

hence

$$
F_{2}^{N, \epsilon}:=\left\{\frac{15}{32} N \epsilon<X_{2}^{N, \epsilon}<\frac{17}{32} N \epsilon\right\}, \quad F_{3}^{N, \epsilon}:=\left\{\frac{7}{32} N \epsilon<X_{3}^{N, \epsilon}<\frac{9}{32} N \epsilon\right\} .
$$

$$
\tilde{Z}^{N, x}(\epsilon) \in \tilde{B}:=\left\{z \in A \mid 1-\frac{17}{32} \epsilon<z_{1}<1-\frac{15}{32} \epsilon, \frac{3}{16} \epsilon<z_{2}<\frac{5}{16} \epsilon\right\}
$$

and

$$
\sup _{t \in[0, \epsilon]}\left|\tilde{Z}^{N, x}(t)-\phi^{x}(t)\right|<\epsilon
$$

on $F_{2}^{N, \epsilon} \cap F_{3}^{N, \epsilon}$. Furthermore, for $z \in \tilde{B}$ and $t \in[0, \eta-\epsilon]$,

$$
\operatorname{dist}\left(\phi^{z}(t), \partial A\right) \geq \frac{3}{16} \epsilon
$$

and

$$
\left|\tilde{Z}^{N, z}(t)-\phi^{z}(t)\right|<\frac{3}{16} \epsilon \Rightarrow\left|\tilde{Z}^{N, z}(t)-\phi^{\tilde{x}}(t)\right|<\epsilon,
$$

where $\tilde{x}=\phi^{x}(\epsilon)=(1-\epsilon / 2, \epsilon / 4)^{\top}$. We compute by using the Markov property of $Z^{N}$,

$$
\begin{aligned}
\mathbb{P}\left[\sup _{t \in[0, \eta]}\left|\tilde{Z}^{N, x}(t)-\phi^{x}(t)\right|<\epsilon\right] \\
\quad \geq \mathbb{P}\left[\sup _{t \in[0, \eta]}\left|\tilde{Z}^{N, x}(t)-\phi^{x}(t)\right|<\epsilon ; F_{2}^{N, \epsilon} \cap F_{3}^{N, \epsilon}\right] \\
\quad \geq \mathbb{P}\left[F_{2}^{N, \epsilon} \cap F_{3}^{N, \epsilon}\right] \cdot \inf _{z \in \tilde{B}} \mathbb{P}\left[\sup _{t \in[0, \eta-\epsilon]}\left|\tilde{Z}^{N, z}(t)-\phi^{z}(t)\right|<\frac{3}{16} \epsilon\right] \\
\geq \mathbb{P}\left[F_{2}^{N, \epsilon} \cap F_{3}^{N, \epsilon}\right] \cdot \inf _{z \in \tilde{B}} \mathbb{P}\left[\sup _{t \in[0, \eta-\epsilon]}\left|\hat{Z}^{N, z}(t)-\phi^{z}(t)\right|<\frac{3}{16} \epsilon\right] \\
\geq 1-\hat{C}_{1} \exp \left(-N \hat{C}_{2}(\epsilon)\right)
\end{aligned}
$$

for appropriate constants $\hat{C}_{1}, \hat{C}_{2}$ with $\hat{C}_{2}(\epsilon)=O\left(\epsilon^{2}\right)$ as $\epsilon \downarrow 0$ by Theorem 3.1 and Inequalities (143) as required. As the rates are vanishing like polynomials, (9) is satisfied.

Our model has a disease-free equilibrium $(0,0)$, and if $\beta>\gamma$ it has a stable endemic equilibrium (and then the disease-free equilibrium is unstable). We assume from now on that $\beta>\gamma$, and we seek to estimate the time it takes for the random perturbations 
to drive our system form the stable endemic equilibrium to the disease-free equilibrium. The characteristic boundary which we want to hit is the set $\{x=0,0 \leq y \leq 1\}$. We note, however, that not only the Assumption 7.1 (D2) but also the Assumption 7.1 (D5) fail to be satisfied here. Consequently we cannot apply Corollary [7.9. We will now show that if we denote by $\tau^{N, x}=\inf \left\{t>0, Z^{N}(t) \in \tilde{O}\right\}$ where $\tilde{O}=\left\{z_{1}=0\right\}$, then Theorem 7.8 applies. All we have to show is that for any $\delta>0, \bar{V}$ being defined as in section 7 ,

$$
\lim _{N \rightarrow \infty} \mathbb{P}\left[e^{(\bar{V}-\delta) N}<\tau^{N, x}<e^{(\bar{V}+\delta) N}\right]=1 .
$$

For any $\eta>0$, let $O_{\eta}=\left\{\left(z_{1}, z_{2}\right) \in[0,1]^{2}, z_{1}>\eta, z_{1}+z_{2} \leq 1\right\}, \tilde{O}_{\eta}=\left\{z_{1}=\eta, 0 \leq z_{2} \leq\right.$ $1-\eta\}$, and $\tau_{\eta}^{N, x}=\inf \left\{t>0, Z^{N}(t) \in \tilde{O}_{\eta}\right\}$. We note that all the above assumptions, including 7.1 (D1), (D2),..., (D5) are satisfied for this new exit problem, so that

$$
\lim _{N \rightarrow \infty} \mathbb{P}\left[e^{\left(\bar{V}_{\eta}-\delta / 2\right) N}<\tau_{\eta}^{N, x}<e^{\left(\bar{V}_{\eta}+\delta / 2\right) N}\right]=1,
$$

where $\bar{V}_{\eta}=\inf _{z \in \tilde{O}_{\eta}} V\left(x^{*}, z\right)$. Now for $\eta_{0}>0$ such that whenever $\eta \leq \eta_{0}, \bar{V}-\delta / 2 \leq$ $\bar{V}_{\eta}<\bar{V}$. Moreover clearly $\tau_{\eta}^{N, x} \leq \tau^{N, x}$. From these follows clearly the fact that $\mathbb{P}\left(\tau^{N, x}>\right.$ $\left.e^{(\bar{V}-\delta) N}\right) \rightarrow 1$ as $N \rightarrow \infty$. It remains to establish the upper bound.

The crucial result which allows us to overcome the new difficulty is the

Lemma 8.1. For any $\eta>0, t>0$,

$$
\liminf _{N \rightarrow \infty} \frac{1}{N} \log \inf _{x \in A^{N} \cap O_{\eta}^{c}} \mathbb{P}\left(\tau^{N, x}<t\right) \geq-\eta \log \left(\frac{\beta}{\gamma}\right) .
$$

Proof. The first component of the process $Z^{N, x}(t)$ is dominated by the process

$$
x_{1}+\frac{1}{N} P_{1}\left(N \beta \int_{0}^{t} Z_{1}^{N, x}(s) d s\right)-\frac{1}{N} P_{1}\left(N \gamma \int_{0}^{t} Z_{1}^{N, x}(s) d s\right),
$$

which is a continuous time binary branching process with birth rate $\beta$ and death rate $\gamma$. This process goes extinct before time $t$ with probability (see the formula in the middle of page 108 in Athreya and Ney [1972])

$$
\left(\frac{\gamma e^{N(\beta-\gamma) t}-N^{-1}}{\beta e^{N(\beta-\gamma) t}-\gamma}\right)^{N x_{1}} .
$$

The result follows readily, since $x \in O_{\eta}^{c}$ implies that $x_{1} \leq \eta$.

In order to adapt the proof of the upper bound in Theorem 7.8 , all we have to do is to extend the proof of Lemma 7.3 to the time of extinction in the SIRS model, which we now do. Indeed, from Lemma [7.3, for any $\eta, \delta, \rho>0$, there exists $T_{0}$ such that

$$
\liminf _{N \rightarrow \infty} \frac{1}{N} \log \underset{x \in \overline{B\left(x^{*}, \rho\right)}}{\inf } \mathbb{P}\left(\tau_{\eta}^{N, x} \leq T_{0}-1\right)>-(\bar{V}+\delta / 2) .
$$

On the other hand, from Lemma 8.1 , provided $\eta<\frac{\delta}{2 \log (\beta / \gamma)}$,

$$
\liminf _{N \rightarrow \infty} \frac{1}{N} \log \inf _{x \in A^{N} \cap O_{\eta}^{c}} \mathbb{P}\left(\tau^{N, x}<1\right) \geq-\delta / 2 .
$$

The statement of Lemma 7.3 now follows from the strong Markov property and the last two estimates. 


\section{A Change of measure}

We assume that $Z^{N, x}=Z^{N}$ has rates $\left\{N \beta_{j} \mid j=1, \ldots, k\right\}$ under $\mathbb{P}$ and rates $\left\{N \tilde{\beta}_{j} \mid j=\right.$ $1, \ldots, k\}$ under $\tilde{\mathbb{P}}$. We furthermore assume that for $x \in A$,

$$
\tilde{\beta}_{j}(x)>0 \text { only if } \beta_{j}(x)>0 \text {. }
$$

Hence, $\left.\tilde{\mathbb{P}}\right|_{\mathcal{F}_{t}}$ is absolutely continuous with respect to $\left.\mathbb{P}\right|_{\mathcal{F}_{+}}$but not necessarily vice versa.

We require Theorem B.6 of Shwartz and Weiss [1995] which gives us an important change of measure formula.

Theorem A.1. For all $T>0$ and non-negative, $\mathcal{F}_{T}$-measurable random variables $X$, we have

$$
\mathbb{E}\left[\xi_{T} X\right]=\tilde{\mathbb{E}}[X]
$$

where $\tilde{\mathbb{E}}$ denotes the expectation with respect to $\tilde{\mathbb{P}}$ and

$$
\begin{aligned}
\xi_{T}:=\exp & \left(\sum _ { \tau } \left[\log \left(\tilde{\beta}_{j(\tau)}\left(Z^{N}(\tau-)\right)\right)-\log \left(\beta_{j(\tau)}\left(Z^{N}(\tau-)\right)\right]\right.\right. \\
& \left.-N \sum_{j} \int_{0}^{T}\left(\tilde{\beta}_{j}\left(Z^{N}(t)\right)-\beta_{j}\left(Z^{N}(t)\right)\right) d t\right) ;
\end{aligned}
$$

here, we sum over the jump times $\tau \in[0, T]$ of $Z^{N} ; j(\tau)$ denotes the corresponding type of the jump direction. In other words, we have

$$
\xi_{T}=\left.\frac{d \tilde{\mathbb{P}}}{d \mathbb{P}}\right|_{\mathcal{F}_{T}} .
$$

We observe that (under $\mathbb{P}) \xi_{T}=0$ if and only if there exists a jump time $\tau \in[0, T]$ (with jump type $j(\tau)$ ) and $\tilde{\beta}_{j(\tau)}\left(Z^{N}(\tau-)\right)=0$.

We deduce the following result. Note that since $\tilde{\mathbb{P}}\left[\xi_{T}=0\right]=0, \xi_{T}^{-1}$ is well-defined $\tilde{\mathbb{P}}$-almost surely.

Corollary A.2. For every non-negative measurable function $X \geq 0$,

$$
\mathbb{E}[X] \geq \tilde{\mathbb{E}}\left[\xi_{T}^{-1} X\right]
$$

Proof. As $X \geq 0$, we have

$$
\mathbb{E}[X] \geq \mathbb{E}\left[X \mathbb{1}_{\left\{\xi_{T} \neq 0\right\}}\right]=\tilde{\mathbb{E}}\left[X \mathbb{1}_{\left\{\xi_{T} \neq 0\right\}} \xi_{T}^{-1}\right]=\tilde{\mathbb{E}}\left[X \xi_{T}^{-1}\right] .
$$

Acknowledgements: This research was supported by the ANR project MANEGE, the DAAD, and the Labex Archimède. 


\section{References}

K. B. Athreya and P. E. Ney. Branching Processes. Springer, New York, 1972.

P. Billingsley. Convergence of Probability Measures. Wiley, New York, 1999.

A. Dembo and O. Zeitouni. Large deviations techniques and applications. Springer, Berlin, 2009.

P. Dupuis and R. S. Ellis. A weak convergence approach to the theory of large deviations. Wiley, New York, 1997.

P. Dupuis, R. S. Ellis, and A. Weiss. Large deviations for Markov processes with discontinuous statistics. I. General upper bounds. Ann. Probab., 19(3):1280-1297, 1991.

J. Feng and T. G. Kurtz. Large deviations for stochastic processes. American Mathematical Society, Providence, 2006.

M. I. Freidlin and A. D. Wentzell. Random Perturbations of Dynamical Systems. Springer, Berlin, 2012.

H. Komiya. Elementary proof for Sion's minimax theorem. Kodai Math. J., 11(1):5-7, 1988.

P. Kratz, E. Pardoux, and B. Samegni Kepgnou. Numerical methods in the context of compartmental models in epidemiology. ESAIM: Proceedings, 48:169-189, 2015.

C. M. Kribs-Zaleta and Velasco-Hernández. A simple vaccination model with multiple endemic states. Math. Biosci., 164(2):183-201, 2000.

T. G. Kurtz. Strong approximation theorems for density dependent Markov chains. Stochastic Processes and their Applications, 6(3):223-240, 1978.

K. Pakdaman, M. Thieullen, and G. Wainrib. Diffusion approximation of birth-death processes: Comparison in terms of large deviations and exit points. Statistics $\&$ Probability Letters, 80(13-14):1121-1127, 2010.

D. Revuz and M. Yor. Continuous Martingales and Brownian Motion. Springer, Berlin, 2005.

H. Roydon. Real Analysis. Collier-Macmillan, London, 1968.

A. Shwartz and A. Weiss. Large Deviations for Performance Analysis. Chapman Hall, London, 1995.

A. Shwartz and A. Weiss. Large deviations with diminishing rates. Mathematics of Operations Research, 30(2):281-310, 2005. 